Dept. of Math. University of Oslo

Pure Mathematics No. 30

ISSN 0806-2439 OCTOBER 2004

\title{
A MODEL OF CONTINUOUS SEDIMENTATION OF FLOCCULATED SUSPENSIONS IN CLARIFIER-THICKENER UNITS
}

\author{
RAIMUND BÜRGER ${ }^{\mathrm{A}}$, KENNETH H. KARLSEN ${ }^{\mathrm{B}}$, AND JOHN D. TOWERS ${ }^{\mathrm{C}}$
}

\begin{abstract}
The chief purpose of this paper is to formulate and partly analyze a new mathematical model for continuous sedimentation-consolidation processes of flocculated suspensions in clarifier-thickener units. This model appears in two variants for cylindrical and variable cross-sectional area units, respectively (Models 1 and 2). In both cases, the governing equation is a scalar, strongly degenerate parabolic equation in which both the convective and diffusion fluxes depend on parameters that are discontinuous functions of the depth variable. The initialvalue problem for this equation is analyzed for Model 1 . We introduce a simple finite-difference scheme and prove its convergence to a weak solution that satisfies an entropy condition. A limited analysis of steady states as desired stationary modes of operation is performed. Numerical examples illustrate that the model realistically describes the dynamics of flocculated suspensions in clarifier-thickeners.
\end{abstract}

\section{INTRODUCTION}

Continuously operated clarifier-thickener units for the solid-liquid separation of suspensions are widely used in chemical engineering, mineral processing, the pulp-and-paper and food industries, and wastewater treatment. For many purposes, spatially one-dimensional mathematical models of these units are sufficient. They are usually based on the kinematic sedimentation theory by Kynch [62], which describes the batch settling of a so-called ideal suspension of small, equal-sized rigid spheres in a viscous fluid by the conservation law $u_{t}+b(u)_{x}=0$ for the solids volume fraction $u$ as a function of depth $x$ and time $t$. The material specific properties of the suspension are described by the Kynch batch flux density function $b(u)$. If a global conservation of mass principle is taken into account, then the extension of this theory to clarifier-thickener units leads to a conservation law with a flux that depends discontinuously on $x$, since the suspension feed flow is split into upwards- and downwards-directed bulk flows into the clarification and thickening zones, respectively. The discontinuous flux makes the well-posedness analysis and numerical simulation of the clarifier-thickener model difficult.

As is well known, the solution of the conservation law arising from the kinematic theory propagates along characteristics, which are straight lines in cylindrical vessels. However, most suspensions are not ideal; rather, they consist of small flocs, or as we say, they are flocculated. These mixtures include inorganic slurries such as tailings from mineral processing, which are flocculated artificially in order to enhance settling rates, as well as biological sludges in wastewater treatment. They form compressible sediment layers, which are characterized by curved iso-concentration lines in settling columns, and can therefore not be predicted by the kinematic theory. Instead, an extended dynamic model including pore pressure and effective solids stress has to be used. Such a model is provided by a theory of sedimentation-consolidation processes [10,27], whose governing

Date: October 7, 2004.

Key words and phrases. Clarifier-thickener units, discontinuous flux, degenerate diffusion, uniqueness, stationary solutions, finite difference scheme, numerical simulation.

Anstitute of Applied Analysis and Numerical Simulation, University of Stuttgart, Pfaffenwaldring 57, D70569 Stuttgart, Germany. E-Mail: buerger@mathematik.uni-stuttgart.de.

${ }^{\mathrm{B}}$ Centre of Mathematics for Applications (CMA), University of Oslo, P.O. Box 1053, Blindern, N-0316 Oslo, Norway. E-Mail: kennethk@math.uio.no.

${ }^{\mathrm{C}}$ MiraCosta College, 3333 Manchester Avenue, Cardiff-by-the-Sea, CA 92007-1516, USA.

E-mail:jtowers@cts. com. 


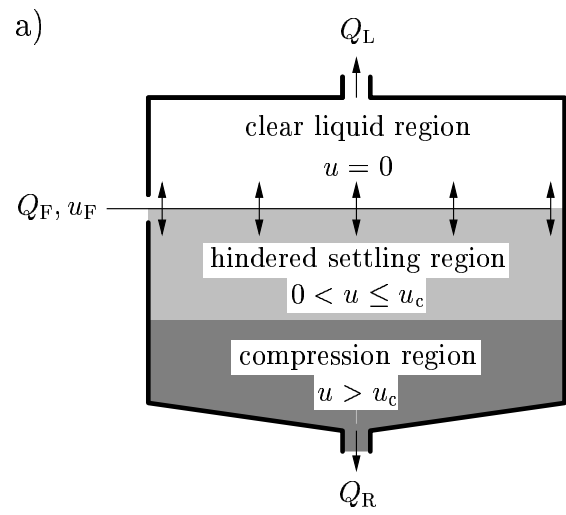

b)

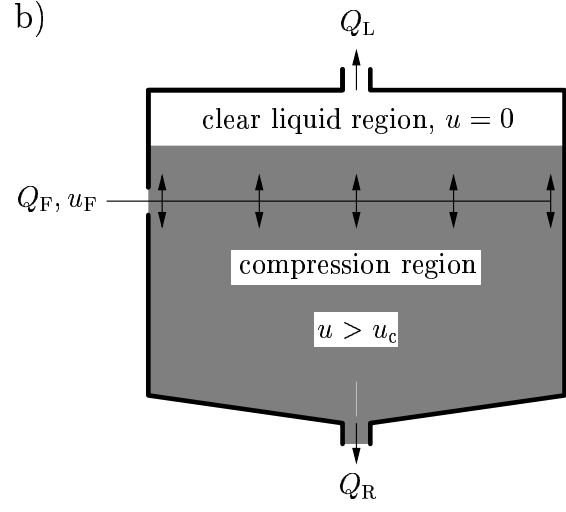

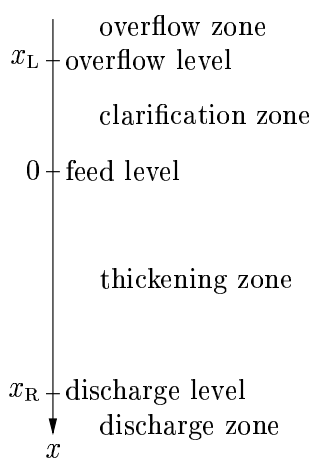

c)

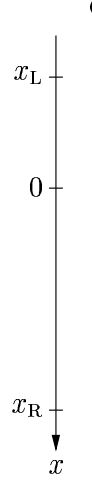

c)

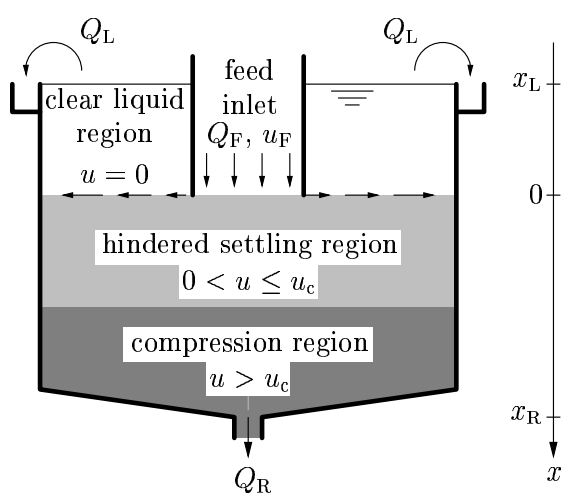

Figure 1. Clarifier-thickener units treating a flocculated suspension: (a) steadystate operation in conventional mode, (b) steady-state operation in high-rate mode, (c) a variant of the clarifier-thickener setup with a vertical feed inlet.

equation (if the model is reduced to one space dimension) is a quasilinear degenerate parabolic equation, which degenerates into the equation of first-order hyperbolic type of the kinematic sedimentation model when $u \leq u_{\mathrm{c}}$, where $u_{\mathrm{c}}$ is a material-dependent critical concentration or gel point at which the solid flocs start to touch each other.

It is the purpose of this paper to present and analyze a clarifier-thickener model for flocculated suspensions as a combination of the first-order models for ideal suspensions with the sedimentationconsolidation theory, which contributes a strongly degenerate diffusion term. The proposed model consists of an initial-value problem for a strongly degenerate parabolic partial differential equation, in which both the convective flux and the diffusion flux depend discontinuously on the spatial variable $x$.

To be more precise, we consider a continuously operated axisymmetric clarifier-thickener vessel as drawn in two variants in Figures 1 (a) and (b) and Figure 1 (c), respectively. Throughout this paper, we assume that all flow variables depend on depth $x$ and time $t$ only. This means in particular that $u$ is assumed to be constant across each horizontal cross section. We subdivide the vessel into four different zones: the thickening zone $\left(0<x<x_{\mathrm{R}}\right)$, which is usually the unique zone considered in conventional analyses of continuous sedimentation, the clarification zone $\left(x_{\mathrm{L}}<\right.$ $x<0)$ located above, the underflow zone $\left(x>x_{\mathrm{R}}\right)$ and the overflow zone $\left(x<x_{\mathrm{L}}\right)$. The vessel is continuously fed at depth $x=0$, the feed level, with fresh feed suspension at a volume feed rate $Q_{\mathrm{F}}(t) \geq 0$. The concentration of the feed suspension is $u_{\mathrm{F}}(t)$. The prescribed volume underflow rate, at which the thickened sediment is removed from the unit, is $Q_{\mathrm{R}}(t) \geq 0$. Consequently, the overflow rate is $Q_{\mathrm{L}}(t)=Q_{\mathrm{R}}(t)-Q_{\mathrm{F}}(t)$, where we assume that the two control functions $Q_{\mathrm{F}}(t)$ and $Q_{\mathrm{R}}(t)$ are chosen such that $Q_{\mathrm{L}}(t) \leq 0$. Of course, the solids concentrations in the underflow and overflow cannot be prescribed, and are part of the solution. Furthermore, we distinguish between 
the four abovementioned zones in the clarifier-thickener, which are a property of the equipment modeled, and the clear liquid, hindered settling, and compression regions, in which a suspension at a given point of time has the concentrations zero, $0<u \leq u_{\mathrm{c}}$, and $u>u_{\mathrm{c}}$, respectively. Thus, the time-dependent location of the regions is a property of a particular flow, that is, of the solution to the problem. Finally, let us mention that the hypothetical assumption $Q_{\mathrm{F}}<0$ would mean that material is suctioned from rather than injected into the unit (as corresponding to our assumption $\left.Q_{\mathrm{F}} \geq 0\right)$. This case is not included in the present analysis.

The model includes two different stationary modes of operation that are usually distinguished in the applicative literature [34]: conventional operation, as shown in Figure 1 (a), when the sediment level (where $u=u_{\mathrm{c}}$ ) is located below the feed level, and high-rate (also known as highcapacity) operation (Figure 1 (b)), when one lets the sediment level (and thus the compression region) rise into the clarification zone. In the latter mode of operation, practitioners observe that the concentration above the compression region usually is zero. These distinctions are made in engineering applications, and we will show that both modes are captured by the model which we analyze in this paper. Figure 1 (c) shows a variant of the clarifier-thickener setup of Figures 1 (a) and (b), in which the feed flow enters the vessel from above through a feed inlet. Note that the feed inlet will usually occupy some of the cross-sectional area of the vessel. We assume that the vessel drawn in Figure 1 (c) is controlled by regulating the feed flow $Q_{\mathrm{F}}$ and the discharge flow $Q_{\mathrm{R}}$, such that no active control of the overflow rate $Q_{\mathrm{L}}$ is necessary. In any circumstance we consider a submerged feed source at a fixed vertical location. The notion "high rate" stems from the observation that this mode of operation usually permits higher solids throughput than the conventional mode, since the clarification zone can handle part of the solids feed flux. Capacity and design calculations based on the new model are, however, outside the scope of this paper. For the sake of simplicity, we also neglect the action of the rake provided in most industrial thickeners, which rotates above the gently sloped floor of the thickener to move the concentrated sediment towards the discharge opening.

Similar clarifier-thickener models were proposed by several authors including Barton et al. [6], Chancelier et al. [30] and Lev et al. [64]. All available treatments are, however, limited to the case of an ideal (non-flocculated) suspension, which is included as a special case in our analysis. In addition, we point out that in [30] the problem of flux discontinuities is circumvented by smoothing out the flux in small $\varepsilon$-neighborhoods of the flux around the levels zero and $x_{\mathrm{R}}$ (in our notation). However, uniqueness for $\varepsilon \rightarrow 0$ is proved in [30] for steady-state solutions only. Important contributions to the analysis and the determination of solutions to clarifier-thickener models for ideal suspensions have been made by Diehl [39, 40, 41, 42, 43], in which local-in-time existence and uniqueness results for problems with piecewise constant initial data are obtained $[39,40,41]$ and stationary solutions are completely classified [41, 43]. Numerical simulations using a Godunov-type scheme are presented in [40, 41, 42]. The paper [34] presents a limited discussion of a steady-state clarifier-thickener model for flocculated suspensions that has many features in common with the one presented here but is incomplete in that boundary conditions or flux transitions at the discharge level are lacking.

In a recent series of papers $[19,21,23,25]$ the authors with collaborators have initiated an activity aiming at providing a firm rigorous ground of mathematical (existence and uniqueness) and numerical analysis for the first-order clarifier-thickener models. Roughly speaking, the main ingredient in these clarifier-thickener models is a first-order scalar conservation law of the type

$$
u_{t}+f(\gamma(x), u)_{x}=0,
$$

where the (with respect to $u$, non-convex) flux $f$ and the discontinuous vector-valued coefficient $\gamma=\left(\gamma_{1}, \gamma_{2}\right)$ are given functions. As is well known, independently of the smoothness of $\gamma(x)$, solutions to (1.1) are in general not smooth and weak solutions must be sought. Moreover, discontinuous weak solutions are in general not uniquely determined by their initial data. Consequently, an entropy condition must be imposed to single out the physically correct solution. These "physically relevant" solutions are called entropy weak solutions. When $\gamma$ is smooth, Kružkov's theory [61] ensures the existence of a unique and stable entropy weak solution to (1.1). Kružkov's theory does not apply when $\gamma$ is discontinuous. In our previous work cited above, which culminated in 
[25], we suggested to use a variant of Kružkov's notion of entropy weak solution for (1.1) that accounts for the discontinuities in $\gamma$. Moreover, we proved existence and uniqueness (stability) of such entropy weak solutions in a certain functional class. The existence of such solutions was a consequence of convergence results for various numerical schemes such as front tracking [19], a relaxation scheme [21], and upwind difference schemes [23, 25].

The papers $[19,21,23,25]$ were inspired by previous work in the area of conservation laws with discontinuous fluxes. Due to their many applications, this is an area that has enjoyed a lot of interest in recent years $[2,5,9,12,39,40,48,49,50,51,52,57,59,60,65,66,67,70,72,73,75$, $76,77,78]$ (this list is not complete). Without entering into too many details, let us just mention that the usual way to cope with the discontinuous parameter $\gamma(x)$ is to express it as an additional conservation law $\gamma_{t}=0$, which yields a system of conservations laws for the "unkowns" $(\boldsymbol{\gamma}, u)$. The equation $\gamma_{t}=0$ introduces linearly degenerate fields in this system with eigenvalues that are zero. Consequently, if $f_{u}$ is zero at some points $(\gamma, u)$, then the system is nonstrictly hyperbolic and it experiences so-called nonlinear resonant behavior, which means more complicated wave interactions than in strictly hyperbolic systems. Indeed, one cannot in general expect to bound the total variation of the conserved quantities directly, but only when measured under a certain singular mapping, as was done first in [76] for a related system. An alternative "scalar" approach in which $\gamma$ is not treated as a separate unknown is presented in $[52,54,55,57,77,78]$, and further developed in $[21,23,25]$ in the context of the first-order clarifier-thickener models. If we took the model studied herein and discretized the discontinuity vector $\gamma(x)$ as an additional conservation law $\gamma_{t}=0$, then we should expect similar nonlinear resonance phenomena as known for first-order systems, since our model degenerates to first-order type on a solution value interval ( $u$-interval) of positive length.

The main ingredient in the models suggested herein, which accounts for compression effects, is not a first-order equation like (1.1), but rather a second-order strongly degenerate parabolic (or mixed hyperbolic-parabolic) equation of the type

$$
u_{t}+f(\gamma(x), u)_{x}=\left(\gamma_{1}(x) A(u)_{x}\right)_{x},
$$

where $A(\cdot)$ is nondecreasing with $A(0)=0$. Note that $A(\cdot)$ can have "flat" regions, and thus (1.2) is strongly degenerate. As a consequence, independently of the smoothness of $\gamma=\left(\gamma_{1}, \gamma_{2}\right)$, solutions to (1.2) will in general be discontinuous and it becomes necessary to work within a framework of entropy weak solutions also for (1.2). In the case of smooth coefficients, the general mathematical theory of hyperbolic conservation laws was developed more than thirty years ago. On the other hand, the mathematical theory for strongly degenerate parabolic equations (with smooth coefficients) has advanced significantly only in the last few years $[7,8,28,31,32,53,68$, $69,82,83,84]$ (this list is not complete either). Although conservation laws with discontinuous fluxes are well studied by now, strongly degenerate parabolic equations with discontinuous fluxes are much less studied. In fact, the only papers that we are aware of are [54, 55, 56], among which the latter two are of importance for the present paper. In [55] equations like (1.2) are studied with a concave convective flux $u \mapsto f(\gamma(x), u)$ and with $\left(\gamma_{1}(x) A(u)_{x}\right)_{x}$ replaced by $A(u)_{x x}$. Existence of an entropy weak solution is established by proving convergence of a difference scheme of the type discussed in this paper. Uniqueness and stability issues for entropy weak solutions are studied in [56] for a particular class of equations.

Herein we develop further the methods used in $[25,55,56]$ in order to apply them to our new mathematical model for the dynamics of flocculated suspensions in clarifier-thickener units. The new results of this paper can be summarized as follows. Firstly, we introduce a suitable definition of entropy weak solutions for the model variant with constant cross-sectional area (to which the mathematical and numerical analysis is limited). We argue that the $x$-discontinuity of the diffusion term $\left(\gamma_{1}(x) A(u)_{x}\right)_{x}$ requires an additional condition in this definition, which states that $A(u)$ is continuous across the jumps of $\gamma_{1}$ (in our model $\gamma_{1}$ is the characteristic function on an interval $\left.\left(x_{\mathrm{L}}, x_{\mathrm{R}}\right)\right)$. Support for the necessity to state this condition comes from analyses of similar equations for two-phase flow in heterogeneous porous media, in which a similar condition is stated, and from the uniqueness analysis of our problem, which is the second novel point and in particular relies on this condition. Thirdly, we formulate a simple finite-difference scheme for 
the clarifier-thickener and prove its convergence by a compactness analysis. A feature of the compactness analysis is that the discontinuities in the fluxes make it hard to bound the total variation of the conserved variable. Instead, we introduce a particular non-linear functional under which we are able to bound the total variation. We show that the limit element satisfies all parts of the definition of entropy weak solutions, except for the continuity of $A(u)$. This issue is left as an open problem. Fourthly, we present an analysis of admissible stationary solutions based on the discussion of entropy weak solutions of the stationary ODE variant of the governing PDE of the transient model, and finally, a limited selection of numerical examples illustrating the clarifierthickener model. Both the steady-state analysis and the numerical simulations support the view that it is reasonable to require $A(u)$ to be continuous.

Before outlining the remainder of this paper, let us briefly mention that we do not explicitly include the effect of hydrodynamic diffusion. This effect is also omitted in the majority of clarifierthickener papers by other authors $[6,30,34,39,40,41,42,43]$, but is included in the analyses by Lev et al. [64] and Verdickt et al. [81]. A profound justification of the omission of hydrodynamic diffusion is beyond the scope of this paper, but is provided in Section 7.4 of [10] on the basis of practical limitations, theoretical considerations, computational comparisons, and experimental results. If we had decided to include hydrodynamic diffusion by adding a term, say, $\mu u_{x x}$ with $\mu>0$ to the right-hand side of (1.2), then the resulting governing PDE would possess smooth solutions and allow for a simpler analytical and numerical treatment than the one advanced in this work. In essence, the discontinuities appearing in transient solutions would be blurred, and in Remark 5.4 we discuss how hydrodynamic affects steady states for the clarifier-thickener problem. Finally, let us mention that hydrodynamic diffusion is not explicitly modelled, but in a sense implicitly present in our model, since our concept of Kružkov entropy weak solution is equivalent to stating that our solution is obtained in the limit $\mu \rightarrow 0$ of smooth solutions of strictly parabolic equations with regularizing (hydrodynamic) diffusion term $\mu u_{x x}$. See Section 4.3.

The remainder of this paper is organized as follows. In Section 2, the clarifier-thickener model is derived. We consider two variants for units with constant and variable interior cross-sectional area, respectively (Models 1 and 2). In particular, we incorporate the governing equation of the sedimentation-consolidation theory developed in full detail in [10]. We describe in Section 3 the finite-difference scheme for the simulation of both models. The scheme appears in two variants, an explicit one which also is analysed, and a semi-implicit one for which a less restrictive condition for the time step size is valid, and which therefore is suitable for large-time simulations. In Section 4 we analyze the initial value problem for Model 1, relying on our previous efforts $[25$, $55,56]$. A definition of entropy weak solutions is given (and discussed extensively), jump and entropy jump conditions are derived, and uniqueness of entropy weak solutions is proved. We study the explicit difference scheme described in Section 3 and prove compactness of a family of approximate solutions generated by this difference scheme. We prove that the limit function $u$ is a weak solution of Model 1 that satisfies the entropy condition. The question whether $A(u)$ is continuous for this limit function is left open. An important problem for practitioners are steadystate solutions, which correspond to the normal operation of a clarifier-thickener unit for constant feed and discharge control parameters. In Section 5, we construct steady-state solutions to Model 1 as piecewise continuous solutions a time-independent ODE version of the transient Model 1. These solutions are again based on the continuity of $A(u)$, but this time this property follows from the ODE formulation. Finally, Section 6 presents a limited choice of numerical examples illustrating Models 1 and 2.

\section{Mathematical Model}

2.1. Balance equations. Consider a vessel with a variable cross-sectional area $S(x)$. Since we assume $u=u(x, t)$, the continuity equations for the solids and the fluid are given by

$$
\begin{aligned}
S(x) u_{t}+\left(S(x) u v_{\mathrm{s}}\right)_{x} & =0, \\
-S(x) u_{t}+\left(S(x)(1-u) v_{\mathrm{f}}\right)_{x} & =0,
\end{aligned}
$$


where $v_{\mathrm{s}}$ and $v_{\mathrm{f}}$ are the solids and the fluid phase velocity, respectively. The mixture flux, that is the volume average flow velocity weighted with the cross-sectional area at height $x$, is given by $Q(x, t):=S(x)\left(u v_{\mathrm{s}}+(1-u) v_{\mathrm{f}}\right)$. The sum of $(2.1)$ and $(2.2)$ produces the continuity equation of the mixture, $Q_{x}(x, t)=0$, which implies that $Q(\cdot, t)$ is constant as a function of $x$. When $Q$ suffers no jumps with respect to $x$, we obtain $Q(x, t)=Q\left(x_{\mathrm{R}}, t\right)=Q(t)$. This equation is equivalent to one of the mass balance equations. We let it replace (2.2) and rewrite (2.1) in terms of the flow rate $Q(t)$ and the solid-fluid relative velocity or slip velocity $v_{\mathrm{r}}:=v_{\mathrm{s}}-v_{\mathrm{f}}$, for which a constitutive equation will be formulated. Observing that

$$
S(x) u v_{\mathrm{s}}=S(x)\left[\left(u v_{\mathrm{s}}+(1-u) v_{\mathrm{f}}\right) u+u(1-u)\left(v_{\mathrm{s}}-v_{\mathrm{f}}\right)\right]=Q(t) u+S(x) u(1-u) v_{\mathrm{r}},
$$

we obtain from (2.1) the equation

$$
S(x) u_{t}+\left(Q(t) u+S(x) u(1-u) v_{\mathrm{r}}\right)_{x}=0 .
$$

The kinematic sedimentation theory [62] is based on the assumption that $v_{\mathrm{r}}$ is a function of $u$ only, $v_{\mathrm{r}}=v_{\mathrm{r}}(u)$. However, the slip velocity is usually expressed in terms of the Kynch batch flux density function $b(u)$, such that $v_{\mathrm{r}}(u)=b(u) /(u(1-u))$ and $(2.4)$ takes the form

$$
S(x) u_{t}+(Q(t) u+S(x) b(u))_{x}=0 .
$$

The function $b$ is usually assumed to be piecewise differentiable with $b(u)=0$ for $u \leq 0$ or $u \geq u_{\max }$, where $u_{\max }$ is the maximum solids concentration, $b(u)>0$ for $0<u<u_{\max }, b^{\prime}(0)>0$ and $b^{\prime}\left(u_{\max }\right) \leq 0$. A typical example is $[74]$

$$
b(u)=v_{\infty} u(1-u)^{C} \quad \text { if } 0<u<u_{\max }, \quad b(u)=0 \quad \text { otherwise, }
$$

where $C \geq 1$ and $v_{\infty}>0$ is the settling velocity of a single floc in pure fluid. It should be pointed out that in the presence of diffusion terms modeling compression effects, to be introduced later, the maximum concentration attained in a settling system depends on the balance between convection and diffusion terms, but not critically on the choice of $u_{\max }$. In order to facilitate the analysis, we assume in this paper $u_{\max }=1$, and that $b(u)$ is smooth on $[0,1]$.

We now apply the sedimentation-consolidation theory outlined in $[10,27]$ to include the sediment compressibility. By constitutive assumptions, a dimensional analysis, and considering one space dimension only, this theory leads to the following equation for the relative velocity $v_{\mathrm{r}}$, which plays the role of one of the linear momentum balances:

$$
v_{\mathrm{r}}=v_{\mathrm{r}}\left(u, u_{x}\right)=\frac{b(u)}{u(1-u)}\left(1+\frac{\sigma_{\mathrm{e}}^{\prime}(u)}{\Delta \varrho g u} u_{x}\right),
$$

where $\Delta \varrho>0$ denotes the solid-fluid density difference, $g$ the acceleration of gravity, and $\sigma_{\mathrm{e}}(u)$ is the effective solid stress function, which is now the second constitutive function (besides $b$ ) characterizing the suspension. This function is assumed to satisfy $\sigma_{\mathrm{e}}(u) \geq 0$ for all $u$ and

$$
\sigma_{\mathrm{e}}^{\prime}(u):=\frac{d \sigma_{\mathrm{e}}(u)}{d u} \begin{cases}=0 & \text { for } u \leq u_{\mathrm{c}}, \\ >0 & \text { for } u>u_{\mathrm{c}} .\end{cases}
$$

A commonly used semi-empirical effective stress formula is the power law

$$
\sigma_{\mathrm{e}}(u)=0 \quad \text { for } u \leq u_{\mathrm{c}} ; \quad \sigma_{\mathrm{e}}(u)=\sigma_{0}\left(\left(u / u_{\mathrm{c}}\right)^{k}-1\right) \quad \text { for } u>u_{\mathrm{c}},
$$

with parameters $\sigma_{0}>0$ and $k>1$. Note that the derivative $\sigma_{\mathrm{e}}^{\prime}(u)$ of the function defined in (2.9) is in general discontinuous at $u=u_{\mathrm{c}}$. Inserting (2.7) into (2.4) and defining

$$
a(u):=\frac{b(u) \sigma_{\mathrm{e}}^{\prime}(u)}{\Delta \varrho g u}, \quad A(u):=\int_{0}^{u} a(s) d s,
$$

we obtain the governing equation

$$
(S(x) u)_{t}+(Q(t) u+S(x) b(u))_{x}=\left(S(x) A(u)_{x}\right)_{x} .
$$

Since $a(u)=0$ for $u \leq u_{\mathrm{c}}$ and $u=u_{\max }$ and $a(u)>0$ otherwise, (2.11) is first-order hyperbolic for $u \leq u_{\mathrm{c}}$ and second-order parabolic for $u>u_{\mathrm{c}}$. Since (2.11) degenerates into hyperbolic type on a solution value interval of positive length, (2.11) is called strongly degenerate parabolic. The location of the type-change interface $u=u_{\mathrm{c}}$ (the sediment level) is in general unknown beforehand. 
For the determination of appropriate functions $b$ and $\sigma_{\mathrm{e}}$ for real materials, see [15, 16, 45]. Moreover, the sedimentation-consolidation model is equivalent to the suspension dewatering theory employed in $[4,38,63,79]$, and other works by the same group of authors.

2.2. The clarifier-thickener model. In the present model, the volume bulk flows are $Q(x, t)=$ $Q_{\mathrm{R}}(t)$ for $x>0$ and $Q(x, t)=Q_{\mathrm{L}}(t)$ for $x<0$. This suggests employing (2.11) with $Q(t)=Q_{\mathrm{R}}(t)$ for $0<x<x_{\mathrm{R}}$ and $Q(t)=Q_{\mathrm{L}}(t)$ for $x_{\mathrm{L}}<x<0$. Furthermore, we assume that in the overflow and underflow zones, the solid material is transported with the same velocity as the liquid. This means that the solid-fluid relative velocity vanishes, $v_{\mathrm{r}}=0$. Moreover, the cross-sectional area $S(x)$ needs to be positive outside the interval $\left[x_{\mathrm{L}}, x_{\mathrm{R}}\right]$. We assume that $S(x)=S_{0}$ for $x<x_{\mathrm{L}}$ and $x>x_{\mathrm{R}}$, where $S_{0}>0$ is a small but positive pipe diameter. From (2.3) we now read off that

$$
\left.S(x) u v_{\mathrm{S}}\right|_{x \notin\left[x_{\mathrm{L}}, x_{\mathrm{R}}\right]}=S_{0} u v_{\mathrm{S}}= \begin{cases}Q_{\mathrm{L}}(t) u & \text { for } x<x_{\mathrm{L}} \\ Q_{\mathrm{R}}(t) u & \text { for } x>x_{\mathrm{R}}\end{cases}
$$

The feed mechanism is introduced by adding a singular source term to the right-hand part of the solids continuity equation (2.1). If we prescribe an initial concentration $u_{0}$ in the vessel, we can summarize the resulting dynamic model as

$$
\begin{gathered}
S(x) u_{t}+\tilde{G}(x, t, u)_{x}=\left(\gamma_{1}(x) A(u)_{x}\right)_{x}+Q_{\mathrm{F}}(t) u_{\mathrm{F}}(t) \delta(x), \quad x \in \mathbb{R}, \quad t>0, \\
u(x, 0)=u_{0}(x), \quad x \in \mathbb{R}, \quad u_{0}(x) \in\left[0, u_{\max }\right], \\
\tilde{G}(x, t, u)=S(x) u v_{\mathrm{s}}= \begin{cases}Q_{\mathrm{L}}(t) u & \text { for } x<x_{\mathrm{L}}, \\
Q_{\mathrm{L}}(t) u+S(x) b(u) & \text { for } x_{\mathrm{L}}<x<0, \\
Q_{\mathrm{R}}(t) u+S(x) b(u) & \text { for } 0<x<x_{\mathrm{R}}, \\
Q_{\mathrm{R}}(t) u & \text { for } x>x_{\mathrm{R}},\end{cases} \\
\gamma_{1}(x):= \begin{cases}S(x) & \text { if } x_{\mathrm{L}} \leq x \leq x_{\mathrm{R}}, \\
0 & \text { otherwise. }\end{cases}
\end{gathered}
$$

For the mathematical analysis we assume that the control functions $Q_{\mathrm{L}}, Q_{\mathrm{R}}$ and $u_{\mathrm{F}}$ are constant. Finally, we may express the singular source term in (2.13) in terms of the derivative of the Heaviside function. Adding the term $-H(x) Q_{\mathrm{F}} u_{\mathrm{F}}$ to $\tilde{G}(x, u)$ and subtracting the term $Q_{\mathrm{L}} u_{\mathrm{F}}$, which is constant with respect to $x$, we obtain the strongly degenerate convection-diffusion problem

$$
\begin{gathered}
S(x) u_{t}+G(x, u)_{x}=\left(\gamma_{1}(x) A(u)_{x}\right)_{x}, \quad x \in \mathbb{R}, \quad t>0, \\
u(x, 0)=u_{0}(x), \quad x \in \mathbb{R}, \quad u_{0}(x) \in\left[0, u_{\max }\right], \\
G(x, u)= \begin{cases}Q_{\mathrm{L}}\left(u-u_{\mathrm{F}}\right) & \text { for } x<x_{\mathrm{L}}, \\
Q_{\mathrm{L}}\left(u-u_{\mathrm{F}}\right)+S(x) b(u) & \text { for } x_{\mathrm{L}}<x<0, \\
Q_{\mathrm{R}}\left(u-u_{\mathrm{F}}\right)+S(x) b(u) & \text { for } 0<x<x_{\mathrm{R}}, \\
Q_{\mathrm{R}}\left(u-u_{\mathrm{F}}\right) & \text { for } x>x_{\mathrm{R}} .\end{cases}
\end{gathered}
$$

2.3. Model 1 (constant interior cross-sectional area). A simple but important sub-case is a vessel whose cross-sectional area is constant in the interior, i.e., we consider

$$
S(x)= \begin{cases}S_{0} & \text { for } x<x_{\mathrm{L}} \text { and } x>x_{\mathrm{R}} \\ S_{\mathrm{int}} & \text { for } x_{\mathrm{L}} \leq x \leq x_{\mathrm{R}}\end{cases}
$$

In this case, the solution of (2.16)-(2.18) does not depend on the value of $S_{0}$. To see this, we introduce the new space variable $w=w(x)$ defined by the bijective mapping $\mathbb{R} \ni x \mapsto w \in \mathbb{R}$,

$$
w(x):= \begin{cases}\left(S_{0} / S_{\text {int }}\right)\left(x-x_{\mathrm{L}}\right)+x_{\mathrm{L}} & \text { for } x<x_{\mathrm{L}} \\ x & \text { for } x_{\mathrm{L}} \leq x \leq x_{\mathrm{R}} \\ \left(S_{0} / S_{\text {int }}\right)\left(x-x_{\mathrm{R}}\right)+x_{\mathrm{R}} & \text { for } x>x_{\mathrm{R}}\end{cases}
$$


and from (2.16) we infer that the function $v$ defined by $v(w(x), t)=u(x, t)$ satisfies the following initial-value problem, where we define the velocities $q_{\mathrm{R}}:=Q_{\mathrm{R}} / S_{\text {int }}, q_{\mathrm{L}}:=Q_{\mathrm{L}} / S_{\text {int }}$, and the diffusion functions $\tilde{a}(\cdot):=a(\cdot) / S_{\mathrm{int}}, \mathcal{A}(\cdot):=A(\cdot) / S_{\mathrm{int}}$ :

$$
\begin{gathered}
v_{t}+g(w, v)_{w}=\left(\gamma_{1}(w) \mathcal{A}(v)_{w}\right)_{w}, \quad w \in \mathbb{R}, \quad t>0, \\
v(w, 0)=u_{0}(w(x)), \quad x \in \mathbb{R}, \\
g(w, v):= \begin{cases}q_{\mathrm{L}}\left(v-u_{\mathrm{F}}\right) & \text { for } w<x_{\mathrm{L}}, \\
q_{\mathrm{L}}\left(v-u_{\mathrm{F}}\right)+b(v) & \text { for } x_{\mathrm{L}}<w<0, \\
q_{\mathrm{R}}\left(v-u_{\mathrm{F}}\right)+b(v) & \text { for } 0<w<x_{\mathrm{R}}, \\
q_{\mathrm{R}}\left(v-u_{\mathrm{F}}\right) & \text { for } w>x_{\mathrm{R}} .\end{cases}
\end{gathered}
$$

We refer to (2.21)-(2.23) as Model 1. Since the variation of $S(x)$ at $x=x_{\mathrm{L}}$ and $x=x_{\mathrm{R}}$ no longer appears in (2.21), Model 1 is formally attained by setting $S \equiv 1$ in (2.18) for all $x \in \mathbb{R}$. This significantly facilitates the analysis. Finally, we define the vector of discontinuity parameters

$$
\gamma:=\left(\gamma_{1}, \gamma_{2}\right), \quad \gamma_{1}(w):=\left\{\begin{array}{ll}
1 & \text { for } w \in\left(x_{\mathrm{L}}, x_{\mathrm{R}}\right), \\
0 & \text { for } w \notin\left(x_{\mathrm{L}}, x_{\mathrm{R}}\right),
\end{array}, \quad \gamma_{2}(w):= \begin{cases}q_{\mathrm{L}} & \text { for } w<0, \\
q_{\mathrm{R}} & \text { for } w>0,\end{cases}\right.
$$

and the flux function

$$
f(\gamma(w), u):=g(x, u)=\gamma_{1}(x) b(u)+\gamma_{2}(x)\left(u-u_{\mathrm{F}}\right) .
$$

Remark 2.1. Consider a non-flocculated ideal suspension, for which $A \equiv 0$. Then Model 1 recovers the clarifier-thickener model with $S \equiv 1$ and $x_{\mathrm{L}}=-x_{\mathrm{R}}$ we analyzed previously [19, 21, 22, 25]. Our derivation now clearly shows that these analyses (including well-posedness and convergence of numerical schemes) are in fact not restricted to the assumption of transport pipes (leading away from the unit for $x<x_{\mathrm{L}}$ and $x>x_{\mathrm{R}}$ ) that have the same diameter as the thickening vessel. Rather, by application of the inverse of (2.20), they are also valid for vessels with cylindrical interior and transport pipes of arbitrarily small (but positive) pipe diameter $S_{0}$.

For the function $b(u)$ given by $(2.6)$ with $v_{\infty}=10^{-4} \mathrm{~m} / \mathrm{s}, C=5$, the velocities $q_{\mathrm{L}}=-10^{-5} \mathrm{~m} / \mathrm{s}$ and $q_{\mathrm{R}}=2.5 \times 10^{-6} \mathrm{~m} / \mathrm{s}$, and $u_{\mathrm{F}}=0.08$, the flux functions $b(u)$ and the fluxes adjacent to the discontinuities of $\gamma$ near $x=x_{\mathrm{L}}, x=0$ and $x=x_{\mathrm{R}}$ are plotted in Figure 2. These parameters will also be utilized in Sections 5 and 6 .

2.4. Model 2 (variable interior cross-sectional area). In the case that $S(x)$ is variable on $\left(x_{\mathrm{L}}, x_{\mathrm{R}}\right)$, we refer to $(2.16)-(2.18)$ as Model 2. It is convenient to rewrite (2.16) as

$$
S(x) u_{t}+F(\gamma(x), u)_{x}=\left(\gamma_{1}(x) A(u)_{x}\right)_{x}
$$

and rewrite the flux function $G(x, u)$ as

$$
F(\gamma(x), u):=G(x, u)=\gamma_{1}(x) b(u)+\gamma_{2}(x)\left(u-u_{\mathrm{F}}\right),
$$

where

$$
\gamma_{1}(w):=\left\{\begin{array}{ll}
S(x) & \text { for } x \in\left(x_{\mathrm{L}}, x_{\mathrm{R}}\right), \\
0 & \text { for } x \notin\left(x_{\mathrm{L}}, x_{\mathrm{R}}\right),
\end{array}, \quad \gamma_{2}(w):= \begin{cases}Q_{\mathrm{L}} & \text { for } x<0 \\
Q_{\mathrm{R}} & \text { for } x>0\end{cases}\right.
$$

\section{NumericAl SCHEME}

The numerical scheme for the solution of $(2.16)-(2.18)$ is a straightforward extension of that used in [23] for the first-order variant of (2.16). To define it, choose $\Delta x>0$, set $x_{j}:=j \Delta x$, and discretize the parameter vector $\gamma$, the initial data, and the cross-sectional area by

$$
\gamma_{j+1 / 2}:=\gamma\left(x_{j+1 / 2}\right), \quad U_{j}^{0}:=u_{0}\left(x_{j}\right), \quad S_{j}:=\frac{1}{\Delta x} \int_{x_{j-1 / 2}}^{x_{j+1 / 2}} S(x) d x .
$$

Here $x_{j+1 / 2}:=x_{j}+\Delta x / 2$, i.e., the midpoint in the interval $\left[x_{j}, x_{j+1}\right)$. In contrast to [25], we discretize $u_{0}$ and $\gamma$ in a pointwise manner, rather than via cell averages. The discretization of $u_{0}$ circumvents some analytical difficulties that would otherwise turn up in the proof of Lemma 4.14, 
a) Kynch batch flux density function $b(u)$

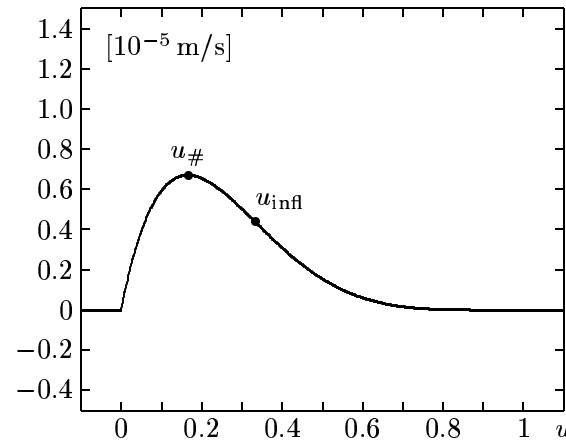

c) Fluxes near $x=x_{\mathrm{R}}$

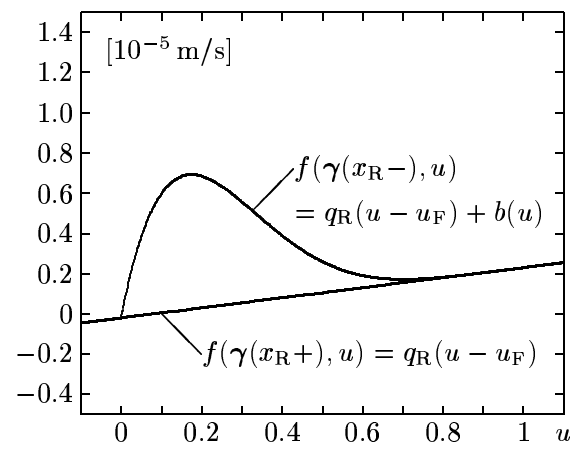

b) Fluxes near $x=x_{\mathrm{L}}$

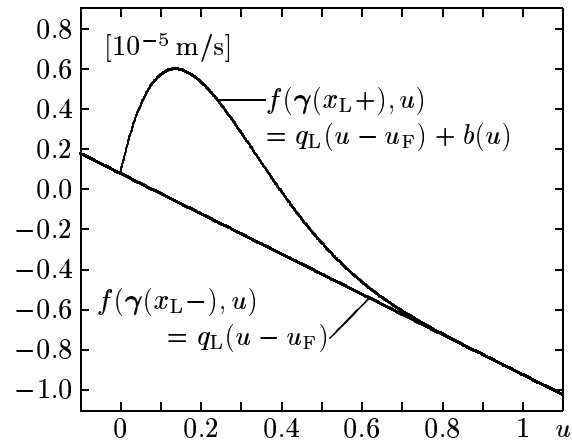

d) Fluxes near $x=0$

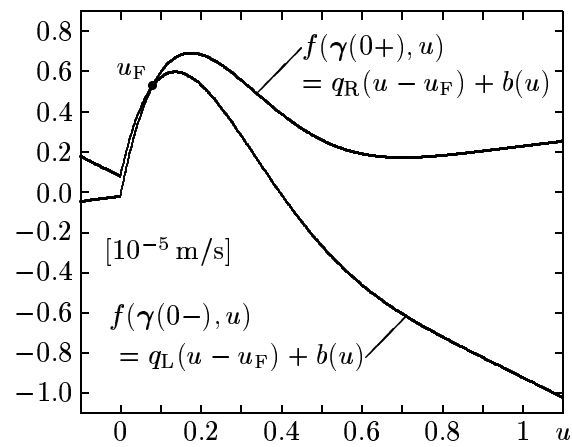

Figure 2. (a) The Kynch batch flux density function $b(u)$ and the fluxes adjacent to (b) $x=x_{\mathrm{L}}$, (c) $x=x_{\mathrm{R}}$ and (d) $x=0$.

and is not unreasonable from a computational point of view. For $n>0$ we define the approximations according to the explicit marching formula

$$
U_{j}^{n+1}=U_{j}^{n}-\lambda_{j} \Delta_{-} h\left(\gamma_{j+1 / 2}, U_{j+1}^{n}, U_{j}^{n}\right)+\frac{\lambda_{j}}{\Delta x} \Delta_{-}\left(\gamma_{1, j+1 / 2} \Delta_{+} A\left(U_{j}^{n}\right)\right),
$$

where $\lambda_{j}:=\Delta t /\left(S_{j} \Delta x\right), \Delta_{-} V_{j}:=V_{j}-V_{j-1}, \Delta_{+} V_{j}:=V_{j+1}-V_{j}$, and

$$
h(\boldsymbol{\gamma}, v, u):=\frac{1}{2}\left[f(\boldsymbol{\gamma}, u)+f(\boldsymbol{\gamma}, v)-\int_{u}^{v}\left|f_{u}(\boldsymbol{\gamma}, w)\right| d w\right]
$$

is the Engquist-Osher flux [44]. Let $t_{n}:=n \Delta t$ and let $\chi^{n}, \chi_{j}$ and $\chi_{j+1 / 2}$ denote the characteristic functions of the intervals $\left[t_{n}, t_{n+1}\right),\left[x_{j-1 / 2}, x_{j+1 / 2}\right)$, and $\left[x_{j}, x_{j+1}\right)$, respectively. We then define

$$
u^{\Delta}(x, t):=\sum_{n \geq 0} \sum_{j \in \mathbb{Z}} U_{j}^{n} \chi_{j}(x) \chi^{n}(t), \quad \gamma^{\Delta}(x):=\sum_{j \in \mathbb{Z}} \gamma_{j+1 / 2} \chi_{j+1 / 2}(x) .
$$

Note that the discontinuity vector $\gamma$ is discretized on a spatial mesh which is staggered (i.e., shifted by $\Delta x / 2$ ) with respect to that of the conserved quantity $u$. This makes it possible to use the scalar Engquist-Osher function (3.2) for the convective part of the problem. A natural alternative would be to align the two discretizations. However, in that case one would have to solve (exactly or approximately) a Riemann problem for a system of two equations in two variables (namely, for $u$ and the volume average velocity $q$ ) at each cell boundary, which makes the resulting numerical method rather complicated, see [48, 59, 60, 65, 66]. In particular, our treatment of the convective part is simpler than the complicated (but accurate) front tracking algorithm used in [19]. Staggering the discretizations also simplifies the analysis, making it possible to apply, with some allowances for the parabolic terms, some of the analytical techniques developed for monotone schemes for purely hyperbolic problems. 
Let us recall that the scheme stated here comprises both Model 1 and Model 2, and is employed for numerical examples for both models in Section 6. The analysis of the scheme is, however, limited to Model 1 (with $S \equiv 1$ ).

In Section 6 we also use the following semi-implicit variant of (3.1) for large-time computations:

$$
U_{j}^{n+1}=U_{j}^{n}-\lambda_{j} \Delta_{-} h\left(\gamma_{j+1 / 2}, U_{j+1}^{n}, U_{j}^{n}\right)+\frac{\lambda_{j}}{\Delta x} \Delta_{-}\left(\gamma_{1, j+1 / 2} \Delta_{+} A\left(U_{j}^{n+1}\right)\right) .
$$

The scheme (3.4) requires the solution of a system of nonlinear equations in each time step by the Newton-Raphson method. This can be done efficiently by Thomas' algorithm, since the coefficient matrix is tridiagonal. The advantage of using (3.4) lies in the fact that we only need to satisfy a CFL condition requiring that $\Delta t / \Delta x$ is bounded, while (3.1) enforces that $\Delta t /(\Delta x)^{2}$ be bounded, see Lemmas 4.12 and 4.13 .

\section{Mathematical analysis}

In several instances we will not repeat arguments that are only minor modifications of proofs that have already appeared in [25], [55] or [56]. In various bounds, $C$ denotes a universal constant.

4.1. The initial value problem. For the sake of consistency with our previous papers, we will abuse the notation slightly by replacing the transformed spatial variable $w$ by $x$, and the transformed functions $v$ and $\mathcal{A}$ by $u$ and $A$, respectively. The Cauchy problem of interest is then

$$
\begin{aligned}
& u_{t}+f(\gamma(x), u)_{x}=\left(\gamma_{1}(x) A(u)_{x}\right)_{x},(x, t) \in \Pi_{T}:=\mathbb{R} \times(0, T) ; \\
& u(x, 0)=u_{0}(x), x \in \mathbb{R},
\end{aligned}
$$

where $f(\gamma, u)=\gamma_{1} b(u)+\gamma_{2}\left(u-u_{\mathrm{F}}\right)$. The parameter vector for this problem is $\gamma:=\left(\gamma_{1}, \gamma_{2}\right)$, where

$$
\gamma_{1}(x):=\left\{\begin{array}{ll}
1 & \text { for } x \in\left(x_{\mathrm{L}}, x_{\mathrm{R}}\right), \\
0 & \text { for } x \notin\left(x_{\mathrm{L}}, x_{\mathrm{R}}\right),
\end{array} \quad \gamma_{2}(x):= \begin{cases}q_{\mathrm{L}} & \text { for } x \leq 0, \\
q_{\mathrm{R}} & \text { for } x>0 .\end{cases}\right.
$$

We assume that $q_{\mathrm{L}}<0$ and $q_{\mathrm{R}}>0$. This rules out the case of batch settling $\left(q_{\mathrm{L}}=0, q_{\mathrm{R}}=0\right)$. However, once our analysis is complete, it will be clear that one can accommodate batch processing as a separate case where one restricts the analysis to the interval $\left[x_{\mathrm{L}}, x_{\mathrm{R}}\right]$. We assume that $b \in C^{2}([0,1])$, and $b(0)=b(1)=0$. Furthermore, we assume that $b^{\prime}$ vanishes at exactly one location $u_{\#} \in(0,1)$, where the function has a maximum, and that $b^{\prime \prime}$ vanishes at no more than one inflection point in $u_{\text {infl }} \in(0,1)$; if such a point is present, we assume that $u_{\text {infl }} \in\left(u_{\#}, 1\right)$. For example, $b(u)$ may be given by (2.6). In accordance with (2.9), (2.10), we will assume that $A \in \operatorname{Lip}([0,1]), A^{\prime}(u)=0$ for $u<u_{\mathrm{c}}$, and that $A^{\prime}(u)>0$ for $u \in\left(u_{\mathrm{c}}, 1\right)$.

For the initial data, we assume that $u_{0}$ satisfies

$$
\left\{\begin{array}{l}
u_{0} \in L^{1}(\mathbb{R}) \cap B V(\mathbb{R}) ; u_{0}(x) \in[0,1] \forall x \in \mathbb{R} ; \\
A\left(u_{0}\right) \text { is absolutely continuous on }\left[x_{\mathrm{L}}, x_{\mathrm{R}}\right] ; \gamma_{1} A\left(u_{0}\right)_{x} \in B V(\mathbb{R}) .
\end{array}\right.
$$

In this paper, $\gamma$ is only allowed to take values in $\mathcal{G}:=\left\{\left(q_{\mathrm{L}}, 0\right),\left(q_{\mathrm{L}}, 1\right),\left(q_{\mathrm{R}}, 0\right),\left(q_{\mathrm{R}}, 1\right)\right\}$. This simplifies matters somewhat compared to our previous paper [25], where the cell average discretization of $\gamma$ required us to consider several lateral sides of the rectangle marked by the vertices in $\mathcal{G}$.

4.2. Definition of entropy weak solution. If (4.1) is allowed to degenerate at certain points, that is, $A^{\prime}(s)=0$ for some values of $s$, solutions are not necessarily smooth and weak solutions must be sought. This property is independent of the smoothness of $\gamma$. Moreover, weak solutions are not necessarily unique, requiring some additional condition, a so-called entropy condition, to single out the physically meaningful solution.

As in [25], the function space $B V_{t}\left(\Pi_{T}\right)$ plays an important role in our definition of entropy weak solution. We denote by $\mathcal{M}\left(\Pi_{T}\right)$ the locally finite Radon (signed) measures on $\Pi_{T}$. The space $B V_{t}\left(\Pi_{T}\right)$ consists of of locally integrable functions $W: \Pi_{T} \rightarrow \mathbb{R}$ for which $\partial_{t} W \in \mathcal{M}\left(\Pi_{T}\right)$.

Let $\mathcal{J}:=\left\{x_{\mathrm{L}}, 0, x_{\mathrm{R}}\right\}$ denote the set of points where $\gamma$ is discontinuous. For a point $m \in \mathcal{J}$, we use the notation $\gamma(m-)$ and $\gamma(m+)$ for the one-sided limits at the point $m$ :

$$
\gamma(m-):=\lim _{x \uparrow m} \gamma(x), \quad \gamma(m+):=\lim _{x \downarrow m} \gamma(x) .
$$


The following definition is motivated by $[25,55,56]$.

Definition 4.1 ( $B V_{t}$ entropy weak solution). A measurable function $u: \Pi_{T} \rightarrow R$ is a $B V_{t}$ entropy weak solution of the initial value problem (4.1) if it satisfies the following conditions:

(D.1) $u \in L^{1}\left(\Pi_{T}\right) \cap B V_{t}\left(\Pi_{T}\right), u \in[0,1]$ a.e. in $\Pi_{T}$, and $A(u)_{x} \in L^{\infty}\left(\left(x_{\mathrm{L}}, x_{\mathrm{R}}\right) \times(0, T)\right)$.

(D.2) For all test functions $\phi \in \mathcal{D}\left(\Pi_{T}\right)$

$$
\iint_{\Pi_{T}}\left(u \phi_{t}+\left[f(\gamma(x), u)-\gamma_{1}(x) A(u)_{x}\right] \phi_{x}\right) d x d t=0 .
$$

(D.3) The initial condition is satisfied in the following strong $L^{1}$ sense:

$$
\underset{t \downarrow 0}{\operatorname{ess} \lim } \int_{\mathbb{R}}\left|u(x, t)-u_{0}(x)\right| d x=0 .
$$

(D.4) For a.e. $t \in[0, T], x \mapsto A(u(x, t))$ is continuous at $x=x_{\mathrm{L}}$ and $x=x_{\mathrm{R}}$.

(D.5) The following Kružkov-type entropy inequality holds for all $c \in \mathbb{R}$ and all test functions $0 \leq \phi \in \mathcal{D}\left(\Pi_{T}\right)$ :

$$
\begin{aligned}
& \iint_{\Pi_{T}}\left(|u-c| \phi_{t}+\operatorname{sgn}(u-c)[f(\gamma(x), u)-f(\gamma(x), c)] \phi_{x}-\gamma_{1}(x)|A(u)-A(c)|_{x} \phi_{x}\right) d x d t \\
& \quad+\int_{0}^{T} \sum_{m \in \mathcal{J}}|f(\gamma(m+), c)-f(\gamma(m-), c)| \phi(m, t) d t \geq 0 .
\end{aligned}
$$

A function $u: \Pi_{T} \rightarrow \mathbb{R}$ satisfying conditions (D.1), (D.2), and (D.3) is called a $B V_{t}$ weak solution of the initial value problem (4.1).

\subsection{Comments on the entropy weak solution concept.}

4.3.1. Motivation of the entropy condition (4.5). It is possible to motivate the entropy condition (4.5) by the fact that limit functions constructed by the method of vanishing viscosity, in combination with smoothing of the coefficients, satisfy this condition. To this end, let $\gamma_{\varepsilon}(x):=\left(\gamma_{1, \varepsilon}, \gamma_{2, \varepsilon}\right)$ be a $C^{\infty}$ approximation of $\gamma$, such that $\gamma_{\varepsilon}$ equals $\gamma$ everywhere except on $(m-\varepsilon, m+\varepsilon)$ for $m \in\left\{x_{\mathrm{L}}, 0, x_{\mathrm{R}}\right\}$, and such that the sign of $\gamma_{\varepsilon}^{\prime}$ is constant on $(m-\varepsilon, m+\varepsilon)$ for $m \in\left\{x_{\mathrm{L}}, 0, x_{\mathrm{R}}\right\}$. Since $\gamma \mapsto f(\gamma, c)$ is linear, $f_{\gamma}(\gamma, c)$ does not depend on $x$. For each $\mu, \varepsilon>0$, let $u^{\mu, \varepsilon}$ be a classical solution to the uniformly parabolic equation

$$
u_{t}^{\mu, \varepsilon}+f\left(\gamma_{\varepsilon}(x), u^{\mu, \varepsilon}\right)_{x}=\left(\gamma_{1, \varepsilon}(x) A\left(u^{\mu, \varepsilon}\right)_{x}\right)_{x}+\mu u_{x x}^{\mu, \varepsilon} .
$$

Let us suppose that $u^{\mu, \varepsilon} \rightarrow u$ in $L_{\text {loc }}^{p}$ for any $1 \leq p<\infty$. The rest of this remark is devoted to proving that the limit $u$ satisfies the entropy condition (4.5).

Since $\gamma_{\varepsilon}$ is smooth, the chain rule and the convexity of $\eta$ imply that $u^{\mu, \varepsilon}$ satisfies the following entropy condition for all convex $C^{2}$ functions $\eta: \mathbb{R} \rightarrow \mathbb{R}$ :

$$
\begin{aligned}
\eta\left(u^{\mu, \varepsilon}\right)_{t} & +q\left(\gamma_{\varepsilon}(x), u^{\mu, \varepsilon}\right)_{x}-\left(\gamma_{1, \varepsilon}(x) r\left(u^{\mu, \varepsilon}\right)_{x}\right)_{x}-\mu \eta\left(u^{\mu, \varepsilon}\right)_{x x} \\
& +\gamma_{\varepsilon}^{\prime}(x) \cdot\left(\eta^{\prime}\left(u^{\mu, \varepsilon}\right) f_{\gamma}\left(\gamma_{\varepsilon}(x), u^{\mu, \varepsilon}\right)-q_{\gamma}\left(\gamma_{\varepsilon}(x), u^{\mu, \varepsilon}\right)\right) \leq 0 \quad \text { in } \mathcal{D}^{\prime}\left(\Pi_{T}\right),
\end{aligned}
$$

where $q: \mathbb{R}^{2} \times \mathbb{R} \rightarrow \mathbb{R}$ and $r: \mathbb{R} \rightarrow \mathbb{R}$ are defined respectively by

$$
q_{u}(\boldsymbol{\gamma}, u)=\eta^{\prime}(u) f_{u}(\boldsymbol{\gamma}, u), \quad r^{\prime}(u)=\eta^{\prime}(u) A^{\prime}(u),
$$

By a standard approximation argument, (4.7) implies that the following Kružkov-type entropy condition holds for any constant $c \in \mathbb{R}$ :

$$
\begin{aligned}
& \left|u^{\mu, \varepsilon}-c\right|_{t}+\left[\operatorname{sgn}\left(u^{\mu, \varepsilon}-c\right)\left(f\left(\gamma_{\varepsilon}(x), u^{\mu, \varepsilon}\right)-f\left(\gamma_{\varepsilon}(x), c\right)\right)\right]_{x}-\left(\gamma_{1, \varepsilon}\left|A\left(u^{\mu, \varepsilon}\right)-A(c)\right|_{x}\right)_{x} \\
& -\mu\left|u^{\mu, \varepsilon}-c\right|_{x}+\operatorname{sgn}\left(u^{\mu, \varepsilon}-c\right) f\left(\gamma_{\varepsilon}(x), c\right)_{x} \leq 0 \quad \text { in } \mathcal{D}^{\prime}\left(\Pi_{T}\right),
\end{aligned}
$$

where

$$
f\left(\gamma_{\varepsilon}(x), c\right)_{x}=\gamma_{\varepsilon}^{\prime}(x) \cdot f_{\gamma}\left(\gamma_{\varepsilon}(x), c\right)=\gamma_{1, \varepsilon}^{\prime}(x) f_{\gamma_{1, \varepsilon}}\left(\gamma_{\varepsilon}(x), c\right)+\gamma_{2, \varepsilon}^{\prime}(x) f_{\gamma_{2, \varepsilon}}\left(\gamma_{\varepsilon}(x), c\right) .
$$


From (4.8) it is clear that the following "less precise" inequality holds:

$$
\begin{aligned}
& \left|u^{\mu, \varepsilon}-c\right|_{t}+\left[\operatorname{sgn}\left(u^{\mu, \varepsilon}-c\right)\left(f\left(\gamma_{\varepsilon}(x), u^{\mu, \varepsilon}\right)-f\left(\gamma_{\varepsilon}(x), c\right)\right)\right]_{x} \\
& -\left(\gamma_{1, \varepsilon}\left|A\left(u^{\mu, \varepsilon}\right)-A(c)\right|_{x}\right)_{x}-\mu\left|u^{\mu, \varepsilon}-c\right|_{x x}-\left|f\left(\gamma_{\varepsilon}(x), c\right)_{x}\right| \leq 0 \quad \text { in } \mathcal{D}^{\prime}\left(\Pi_{T}\right),
\end{aligned}
$$

that is, for any $0 \leq \phi \in \mathcal{D}^{\prime}\left(\Pi_{T}\right)$,

$$
\begin{gathered}
\iint_{\Pi_{T}}\left(\left|u^{\mu, \varepsilon}-c\right| \phi_{t}+\operatorname{sgn}\left(u^{\mu, \varepsilon}-c\right)\left(f\left(\gamma_{\varepsilon}(x), u^{\mu, \varepsilon}\right)-f\left(\gamma_{\varepsilon}(x), c\right)\right) \phi_{x}\right. \\
\left.-\gamma_{1, \varepsilon}\left|A\left(u^{\mu, \varepsilon}\right)-A(c)\right|_{x} \phi_{x}+\mu\left|u^{\mu, \varepsilon}-c\right| \phi_{x x}\right) d x d t \\
+\iint_{\Pi_{T}}\left|f\left(\gamma_{\varepsilon}(x), c\right)_{x}\right| \phi d x d t \geq 0 .
\end{gathered}
$$

Using the properties of $\gamma$ and $\gamma_{\varepsilon}$, we can write (notice the signs)

$$
\begin{aligned}
& \iint_{\Pi_{T}}\left|f\left(\gamma_{\varepsilon}(x), c\right)_{x}\right| \phi d x d t=\int_{0}^{T} \int_{x_{\mathrm{L}}-\varepsilon}^{x_{\mathrm{L}}+\varepsilon} \gamma_{1, \varepsilon}^{\prime}(x)|b(c)| \phi(x, t) d x d t \\
& \left.\quad-\int_{0}^{T} \int_{x_{\mathrm{R}}-\varepsilon}^{x_{\mathrm{R}}+\varepsilon} \gamma_{1, \varepsilon}^{\prime}(x)|b(c)| \phi(x, t) d x d t+\int_{0}^{T} \int_{-\varepsilon}^{\varepsilon} \gamma_{2, \varepsilon}^{\prime}(x) \mid c-u_{\mathrm{F}}\right) \mid \phi(x, t) d x d t .
\end{aligned}
$$

After three integrations by parts and subsequently sending $\varepsilon$ to zero, we obtain

$$
\begin{aligned}
\lim _{\varepsilon \rightarrow 0} \iint_{\Pi_{T}}\left|f\left(\gamma_{\varepsilon}(x), c\right)_{x}\right| \phi d x d t=\int_{0}^{T} & \left(\gamma_{1}\left(x_{\mathrm{L}}+\right)-\gamma_{1}\left(x_{\mathrm{L}}-\right)\right)|b(c)| \phi\left(x_{\mathrm{L}}, t\right) d t \\
& -\int_{0}^{T}\left(\gamma_{1}\left(x_{\mathrm{R}}+\right)-\gamma_{1}\left(x_{\mathrm{R}}-\right)\right)|b(c)| \phi\left(x_{\mathrm{R}}, t\right) d t \\
& \left.\quad+\int_{0}^{T}\left(\gamma_{2}(0+)-\gamma_{2}(0-)\right) \mid c-u_{\mathrm{F}}\right) \mid \phi(0, t) d t \\
= & \int_{0}^{T} \sum_{m \in \mathcal{J}}|f(\gamma(m+), c)-f(\gamma(m-), c)| \phi(m, t) d t .
\end{aligned}
$$

In view of the previous calculations, we can send $\mu, \varepsilon \rightarrow 0$ in (4.10) to conclude that the limit function $u$ satisfies the entropy condition (4.5).

4.3.2. Status of the weak formulation (4.3). We point out that the entropy inequality (4.5) does not imply the weak formulation (4.3). In fact, the usual procedure to derive the weak formulation from the Kružkov entropy inequality consists in taking first $c>\|u\|_{\infty}$ and then $c<-\|u\|_{\infty}$, and then combining the resulting inequalities. This does not work here, since the term $\mid f(\gamma(m+), c)-$ $f(\gamma(m-), c) \mid$ does not have compact support with respect to $c$, and therefore the sum over $m \in \mathcal{J}$ in (4.5) will not disappear for these values of $c$. Consequently, we state the weak formulation (4.3) and the entropy inequality (4.5) as independent ingredients of the solution concept.

4.3.3. Alternative entropy weak solution concepts. Section 4.3.1 shows that the (Kružkov) entropy formulation (4.5) for the clarifier-thickener model can be derived from the parabolic regularization (4.6) when the regularization parameter $\mu$ and the parameter $\varepsilon$ that smoothes the flux discontinuities tend to zero. In Section 1 we mentioned that the term $\mu u_{x x}$ corresponds to hydrodynamic diffusion. Consequently, the entropy formulation (4.5), which eventually leads to uniqueness, encodes that our 'physically relevant' solutions are those obtained in the limit of vanishing hydrodynamic diffusion.

Alternative entropy concepts for conservation laws with discontinuous flux, which equally lead to unique solutions (that are, however, different from the ones constructed here) are possible. For example, a mathematical model for two-phase flow in a one-dimensional porous medium that changes its type at $x=0$ can be written as the conservation law with discontinuous flux

$$
u_{t}+(H(x) f(u)+(1-H(x)) g(u))_{x}=0,
$$


where $u$ is the saturation of one of the phases, the functions $f(u)$ and $g(u)$ are the rock-type dependent Darcy velocities for $x>0$ and $x<0$, respectively, and $H(x)$ is the Heaviside function. These models are usually based on the assumption that the capillary pressure is continuous across heterogeneities of the porous medium. Consequently, the appropriate viscous regularization term of (4.11) for this model is not given by $\mu u_{x x}^{\varepsilon}$, but by $\mu\left(\lambda_{c}\left(u^{\varepsilon}\right) p_{c}\left(u^{\varepsilon}\right)\right)$, where $\lambda_{c}$ and $p_{\mathrm{c}}$ are the mobility and capillary pressure functions for the phase considered and $x<0(c=\mathrm{L})$ and $x>0$ $(c=\mathrm{R})$, respectively [51, 71]. Analyzing the limit $\varepsilon \rightarrow 0$ for this regularization term, Kaasschieter [51] shows that the corresponding viscosity limit produces an entropy condition for the limiting equation (4.11) that excludes that characteristics leave the discontinuity at $x=0$ from both sides. In other words, the capillary pressure characterization does not allow undercompressive shocks emerging from $x=0$. This contrasts with the role of the flux discontinuity at $x=0$ in the clarifier-thickener model, in which material is injected at $x=0$ and transported from both sides of that source term location, which allows that characteristics emerge to both sides of $x=0$.

Based on the properties of this two-phase flow in heterogeneous porous media model, Adimurthi, Jaffré and Veerrappa Gowda propose in [1] an entropy formulation alternative to ours, which is essentially based on the usual Kružkov entropy characterization away from flux discontinuities, supplemented by an additional entropy jump condition that rules out undercompressive shocks. In [1] it is shown that regular solutions satisfying these conditions are unique, and convergence of a Godunov-type scheme to weak solutions of this type is proved. The entropy concepts of [1] and [25] (to which the present treatment reduces for $A \equiv 0$ ) coincide if the 'left' and 'right' fluxes $f(u)$ and $g(u)$ do not intersect, and in general yield different results if these fluxes intersect in an 'undercompressive' way. This means that if we suppose, for simplicity, that the functions $f(u)$ and $g(u)$ intersect at only one point, called $u_{\chi}$ here, then in the vicinity of $u_{\chi}$, we have $g^{\prime}(u)<0$ for $u \leq u_{\chi}$ and $f^{\prime}(u)>0$ for $u \geq u_{\chi}$. Such a situation frequently occurs in the two-phase flow in porous media model, and is also possible in the clarifier-thickener model. For example, consider the situation near the feed level $x=0$, and assume that we take the parameters $q_{\mathrm{L}}$ and $q_{\mathrm{R}}$ as used in Figure 2, but set $u_{\mathrm{F}}=0.8$. Graphically, this situation corresponds to moving the two curves in Figure 2 (d) further apart until they intersect at $u_{\chi}=u_{\mathrm{F}}=0.8$. From the shape of this curves it becomes clear that this is indeed a case of an 'undercompressive' intersection, where $f(u)=q_{\mathrm{R}}\left(u-u_{\mathrm{F}}\right)+b(u)$ and $g(u)=q_{\mathrm{L}}\left(u-u_{\mathrm{F}}\right)+b(u)$. Furthermore, if we assume that the clarifier-thickener is initially filled with suspension of that same concentration $u_{0}=u_{\mathrm{F}}$, we expect that filling up the vessel with (strongly concentrated) suspension of concentration $u=u_{\mathrm{F}}$ produces a solution that is constant near $x=0$ (for small times at least, until waves coming from $x_{\mathrm{L}}$ or $x_{\mathrm{R}}$ start to interfere). In this scenario, however, the theory of [1], which excludes undercompressive shocks, leads to a different solution, which includes one stationary oscillation between solution values $\theta_{f}$ and $\theta_{g}$ such that $f^{\prime}\left(\theta_{f}\right) \leq 0$ or $g^{\prime}\left(\theta_{g}\right) \geq 0$, i.e., the resulting stationary discontinuity is not undercompressive. In [20] we also present a numerical example for a similar case showing that both entropy theories, along with the convergence of the respective associated schemes, lead to different admissible solutions. Of course, these alternative theories mirror the different physics involved, as the different viscous regularizations of the clarifier-thickener model and of the two-phase flow in porous media model show. Finally, let us clarify that the problem of whether the fluxes $f(u)$ and $g(u)$ intersect in an 'undercompressive way' is not equivalent to the satisfaction of the so-called "crossing condition", to which we appeal in Section 4.5 for the uniqueness proof. See the comment following Lemma 4.11 for a discussion of this point in the context of the clarifier-thickener model.

4.3.4. Motivation of condition (D.4). One consequence of this definition is that for a.e. $t \in[0, T]$, $A(u(x, t))$ is absolutely continuous as a function of $x$ on $\left[x_{\mathrm{L}}, x_{\mathrm{R}}\right]$ and that $A(u)_{x}$ exists a.e. in $\left[x_{\mathrm{L}}, x_{\mathrm{R}}\right]$. The point that deserves to be discussed extensively is, however, our explicit requirement that $A(u)$ be continuous across $x=x_{\mathrm{L}}$ and $x=x_{\mathrm{R}}$. To justify this assumption, we follow the analysis of van Duijn et al. [80] and Molenaar [71]. We consider the situation near $x_{\mathrm{R}}$ (the jump across $x_{\mathrm{L}}$ is handled in the same way). We approximate the discontinuous coefficient $\gamma_{1}(x)$ by a smooth function $\gamma_{1}^{\varepsilon}(x)$, where $\varepsilon>0$ is a small smoothing parameter and $\gamma_{1}^{\varepsilon}(x)=\gamma_{1}(x)$ outside $\left(x_{\mathrm{R}}-\varepsilon, x_{\mathrm{R}}+\varepsilon\right)$. The differential equation that should be satisfied by the limit function in this 
interval for $\varepsilon \rightarrow 0$ provides an additional interface condition. We assume that $v^{\varepsilon}$ is the solution of Model 1 with $\gamma_{1}(x)$ replaced by $\gamma_{1}^{\varepsilon}$, and introduce the the rescaled space variable $y:=\left(x-x_{\mathrm{R}}\right) / \varepsilon$. Inside $\left(x_{\mathrm{R}}-\varepsilon, x_{\mathrm{R}}+\varepsilon\right)$, our equation can be written as $\varepsilon v_{t}^{\varepsilon}+\left(f^{\varepsilon}\left(y, v^{\varepsilon}\right)\right)_{y}=0$ with

$$
f^{\varepsilon}\left(y, v^{\varepsilon}\right)=q_{\mathrm{R}}\left(v^{\varepsilon}-u_{\mathrm{F}}\right)+\gamma_{1}^{\varepsilon} b\left(v^{\varepsilon}\right)-\gamma_{1}^{\varepsilon} \frac{1}{\varepsilon} A\left(v^{\varepsilon}\right)_{y} .
$$

We assume that $v^{\varepsilon}$ with $0 \leq v^{\varepsilon} \leq 1$ converges to a function $v$ as $\varepsilon \rightarrow 0$, and that the total flux $f^{\varepsilon}$ remains bounded uniformly in $\varepsilon$ as $\varepsilon \rightarrow 0$. Then the limit function $v$ should satisfy $A(v)_{y}=0$ for $-1<y<1$. Integrating this equation yields the following relation between $v(-1, t)$ and $v(1, t)$, which are identified with the limits of $u$ to the left and to right of $x=x_{\mathrm{R}}, u\left(x_{\mathrm{R}}-, t\right)$ and $u\left(x_{\mathrm{R}}+, t\right)$ :

$$
A\left(u\left(x_{\mathrm{R}}+, t\right)\right)-A\left(u\left(x_{\mathrm{R}}-, t\right)\right)=A(v(1, t))-A(v(-1, t))=\int_{-1}^{1} A(v)_{y} d y=0,
$$

which motivates our condition (D.4).

To highlight the status of condition (D.4), we first mention that (D.4) is the analogue of the 'extended pressure condition' postulated in problems of multiphase flow in heterogeneous porous media $[71,80,85]$. These problems lead to equations with discontinuous flux and discontinuous (with respect to the space variable) diffusion, which require an additional jump condition across jumps of the diffusion coefficient (apart from the appropriate Rankine-Hugoniot condition) to ensure uniqueness. This analogy, and the observation that in our case, the diffusion terms are not present at all for $x<x_{\mathrm{L}}$ and $x>x_{\mathrm{R}}$ and it is therefore unlikely to obtain control on the limits of $A(u)_{x}$ for $x \downarrow x_{\mathrm{L}}$ and $x \uparrow x_{\mathrm{R}}$, strongly support the necessity to postulate (D.4) as a separate ingredient of the definition of an entropy weak solution. This view is also supported by the fact that our uniqueness proof relies on (D.4). In other words, (D.4) is necessary to ensure uniqueness.

It should be mentioned, however, that it is currently unclear how to prove that the numerical scheme converges to a solution that satisfies (D.4). In fact, we will prove that the scheme converges to a limit $u$ that satisfies all components of Definition 4.1 except (D.4). However, our numerical results support that $A(u)$ is continuous across $x=x_{\mathrm{L}}$ and $x=x_{\mathrm{R}}$. In particular, transient numerical simulations converge (for large times) to steady-state solutions. These are $x$-dependent piecewise continuous functions that are defined by a time-independent version of Definition 4.1 which does not include a postulate of continuity of $A(u)$, since satisfaction of this condition can be derived in the ODE context.

4.4. Existence of traces. In what follows, we often use the notation

$$
F(\boldsymbol{\gamma}, u, c):=\operatorname{sgn}(u-c)(f(\boldsymbol{\gamma}, u)-f(\boldsymbol{\gamma}, c))
$$

for the Kružkov entropy flux appearing in (4.5). Here, the sign function is defined $\operatorname{by} \operatorname{sgn}(w):=$ $w /|w|$ for $w \neq 0$ and $\operatorname{sgn}(0):=0$. It is sometimes convenient to work with the function $\hat{f}(\gamma, u):=$ $f(\boldsymbol{\gamma}, u)+\gamma_{2} u_{\mathrm{F}}$, and to use the identity $F(\boldsymbol{\gamma}, u, c)=\operatorname{sgn}(u-c)(\hat{f}(\boldsymbol{\gamma}, u)-\hat{f}(\boldsymbol{\gamma}, c))$. We will also find the following notation useful: $a \vee b:=\max \{a, b\}, a \wedge b:=\min \{a, b\}, a_{+}:=a \vee 0$, and $a_{-}:=a \wedge 0$.

Our analysis makes use of certain jump conditions that relate limits from the right and left of the entropy weak solution $u$ at jumps in the spatially varying coefficient $\gamma(x)$. Thus, we need a notion of one-sided limits (from both the right and left) at the points $x \in \mathcal{J}$.

Definition 4.2 (Traces). Let $W \in L^{\infty}\left(\Pi_{T}\right)$ be a real function. By the right and left traces of $W(\cdot, t)$ at a point $x=x_{0} \in \mathbb{R}$ we understand functions $t \mapsto W\left(x_{0} \pm, t\right) \in L^{\infty}(0, T)$ that satisfy

$$
\begin{array}{ll}
\underset{x \downarrow x_{0}}{\operatorname{ess} \lim }\left|W(x, t)-W\left(x_{0}+, t\right)\right|=0 & \text { for a.e. } t \in(0, T), \\
\underset{x \uparrow x_{0}}{\operatorname{ess} \lim }\left|W(x, t)-W\left(x_{0}-, t\right)\right|=0 & \text { for a.e. } t \in(0, T) .
\end{array}
$$

As in $[25,56]$, we now derive the existence of the required traces from our definition of $B V_{t}$ entropy weak solution. To this end, we introduce the so-called singular mapping. Let $\mathcal{S}(w)$ denote the characteristic function of the interval $\left[0, u_{\mathrm{c}}\right]$, where $A^{\prime}(u)=0$, and recall that

$$
f(\gamma(w), u)=\gamma_{1}(x) b(u)+\gamma_{2}(x)\left(u-u_{\mathrm{F}}\right) .
$$


The singular mapping is defined by

$$
\Psi(\gamma, u):=\mathcal{F}(\gamma, u)+\gamma_{1} A(u), \quad \mathcal{F}(\gamma, u):=\int_{0}^{u}\left(\gamma_{1} \mathcal{S}(w)+1-\gamma_{1}\right)\left|f_{u}(\gamma, w)\right| d w .
$$

Following [55], we have broken the singular mapping into two parts, $\mathcal{F}$ for the hyperbolic spatial operator and $\gamma_{1} A$ for the parabolic operator. Note that if $\gamma_{1}=0$, which means that $x \notin\left[x_{\mathrm{L}}, x_{\mathrm{R}}\right]$, the parabolic term will not be present and the singular mapping simplifies to $\Psi(\gamma, u)=\mathcal{F}(\gamma, u)=$ $\int_{0}^{u}\left|f_{u}(\gamma, w)\right| d w$. If $x \in\left(x_{\mathrm{L}}, x_{\mathrm{R}}\right)$, then $\gamma_{1}=1$, and

$$
\Psi(\gamma, u)=\mathcal{F}(\gamma, u)+A(u), \quad \mathcal{F}(\gamma, u)=\int_{0}^{u} \mathcal{S}(w)\left|f_{u}(\gamma, w)\right| d w .
$$

Thus, for $x \in\left(x_{\mathrm{L}}, x_{\mathrm{R}}\right)$,

$$
\partial_{u} \Psi(\gamma, u)= \begin{cases}\left|f_{u}(\gamma, u)\right| & \text { for } u \leq u_{\mathrm{c}}, \\ A^{\prime}(u) & \text { for } u>u_{\mathrm{c}} .\end{cases}
$$

Thus, we see that for any fixed value of $x$ (and hence $\gamma$ ) and $u$, exactly one of the mappings $u \mapsto \mathcal{F}(\gamma, u), u \mapsto \gamma_{1} A(u)$ is increasing, and the other is constant. This allows us to separate the hyperbolic and parabolic terms somewhat in our analysis, and is the motivation behind the particular form of the singular mapping given by (4.13).

The following lemma records some easily verified properties of $\Psi$. We omit the elementary proofs.

Lemma 4.1. The mapping $u \mapsto \Psi(\gamma, u)$ is Lipschitz continuous on $[0,1]$, uniformly for $\gamma \in \mathcal{G}$. In addition, $u \mapsto \Psi(\gamma, u)$ is strictly increasing on $[0,1]$ for each fixed vector $\gamma \in \mathcal{G}$.

The proof of the following lemma is easily adapted to the present situation from that of Lemma 3.1 of [56]. The key ingredients are (4.3), (4.5) and the fact that $u_{t} \in \mathcal{M}\left(\Pi_{T}\right)$.

Lemma 4.2. Suppose $u$ is a $B V_{t}$ entropy weak solution. Then, for any $c \in \mathbb{R}$,

$$
\begin{gathered}
\left(f(\gamma(x), u)-f(\gamma(x), c)-\gamma_{1}(x)(A(u)-A(c))_{x}\right)_{x} \in \mathcal{M}\left(\Pi_{T}\right), \\
\left(\operatorname{sgn}(u-c)[f(\gamma(x), u)-f(\gamma(x), c)]-\gamma_{1}(x)|A(u)-A(c)|_{x}\right)_{x} \in \mathcal{M}\left(\Pi_{T}\right) .
\end{gathered}
$$

Lemma 4.3. Let $u$ be a $B V_{t}$ entropy weak solution of (4.1), and consider the transformed function $z(x, t):=\Psi(\gamma(x), u(x, t))$. Then $\int_{0}^{T} \operatorname{TV}(z(\cdot, t)) d t<C$ for some finite constant $C>0$. In other words, $z_{x} \in \mathcal{M}\left(\Pi_{T}\right)$.

Proof. For $A \equiv 0$, the proof of Lemma 2.2 of [25] applies unchanged up to minor differences in notation. We here modify that proof to account for the presence of $A(u)$. Let $\operatorname{TV}\left(\left.z(\cdot, t)\right|_{\mathcal{I}}\right)$ denote the spatial variation of $z(\cdot, t)$ measured over the interval $\mathcal{I}$. Then it suffices to show that $\int_{0}^{T} \operatorname{TV}\left(\left.z(\cdot, t)\right|_{\mathcal{I}}\right) d t$ is bounded for each of the open intervals $\mathcal{I}=\left(-\infty, x_{\mathrm{L}}\right),\left(x_{\mathrm{L}}, 0\right),\left(0, x_{\mathrm{R}}\right)$, and $\left(x_{\mathrm{R}}, \infty\right)$. Due to the factor $\gamma_{1}(x)$, the singular mapping $\Psi$ simplifies to $\Psi(\boldsymbol{\gamma}, u)=\int_{0}^{u}\left|f_{u}(\boldsymbol{\gamma}, w)\right| d w$ when $\mathcal{I}=\left(-\infty, x_{\mathrm{L}}\right)$ or $\mathcal{I}=\left(x_{\mathrm{R}}, \infty\right)$, and so the proof of Lemma 2.2 of [25] applies to those intervals without any modifications. We will focus on the interval $\mathcal{I}=\left(0, x_{\mathrm{R}}\right)$, and the omit the proof for $\left(x_{\mathrm{L}}, 0\right)$ since it is similar. Thus, we now set out to show that

$$
\int_{0}^{T} \operatorname{TV}\left(\left.z(\cdot, t)\right|_{\left\{x \mid 0<x<x_{\mathrm{R}}\right\}}\right) d t<\infty .
$$

To this end, recall that for $x \in\left(0, x_{\mathrm{R}}\right)$, we have $\gamma=\left(1, q_{\mathrm{R}}\right)$, and

$$
\begin{aligned}
f(\boldsymbol{\gamma}, u) & =q_{\mathrm{R}} u+b(u)-q_{\mathrm{R}} u_{\mathrm{F}}=\hat{f}(\boldsymbol{\gamma}, u)-q_{\mathrm{R}} u_{\mathrm{F}}, \\
\Psi(\boldsymbol{\gamma}, u) & =\int_{0}^{u} \mathcal{S}(w)\left|\hat{f}_{u}(\boldsymbol{\gamma}, w)\right| d w+A(u)=\int_{0}^{u} \mathcal{S}(w)\left|q_{\mathrm{R}}+b^{\prime}(w)\right| d w+A(u), \\
F(\boldsymbol{\gamma}, u, c) & =\operatorname{sgn}(u-c)(f(\boldsymbol{\gamma}, u)-f(\boldsymbol{\gamma}, c))=\operatorname{sgn}(u-c)(\hat{f}(\boldsymbol{\gamma}, u)-\hat{f}(\boldsymbol{\gamma}, c)) .
\end{aligned}
$$

Let $\Pi_{T}^{\mathrm{R}}:=\left(0, x_{\mathrm{R}}\right) \times(0, T) \subset \Pi_{T}$. Since $A(u)_{x} \in L^{\infty}\left(\left(x_{\mathrm{L}}, x_{\mathrm{R}}\right) \times(0, T)\right)$, we have

$$
A(u)_{x} \in \mathcal{M}\left(\Pi_{T}^{\mathrm{R}}\right), \quad(A(u)-A(c))_{x} \in \mathcal{M}\left(\Pi_{T}^{\mathrm{R}}\right), \quad|A(u)-A(c)|_{x} \in \mathcal{M}\left(\Pi_{T}^{\mathrm{R}}\right) .
$$


Thus, it suffices to show that $\mathcal{F}(\gamma, u)_{x} \in \mathcal{M}\left(\Pi_{T}^{\mathrm{R}}\right)$. Note that

$$
\mathcal{F}(\gamma(x), u(x, t))=\int_{0}^{u(x, t)} \mathcal{S}(w)\left|\hat{f}_{u}(\gamma, w)\right| d w \quad \text { for } \quad(x, t) \in \Pi_{T}^{\mathrm{R}} .
$$

Due to the assumptions on $q_{\mathrm{R}}$ and $b(u)$, the function $\hat{f}$ has at most two extrema for $u \in(0,1)$. We assume that $q_{\mathrm{R}}$ is chosen such that there are exactly two extrema $u_{1}^{*}<u_{2}^{*}$. The cases with one or no extremum, will be omitted; they are handled in a similar manner. It is clear that $u \mapsto \hat{f}(\boldsymbol{\gamma}, u)$ is strictly monotone on intervals not containing extrema. We need the following fact, which follows by subtracting (4.14) from (4.15), and then dividing by 2 .

$$
\left(\chi_{l}(w ; c)\left(f\left(\gamma_{\mathrm{R}}, u\right)-f\left(\gamma_{\mathrm{R}}, c\right)\right)-\gamma_{1}(x)\left((A(u)-A(c))_{-}\right)_{x}\right)_{x} \in \mathcal{M}\left(\Pi_{T}^{\mathrm{R}}\right), \quad \forall c \in \mathbb{R} .
$$

Here $\chi_{l}(w ; c)$ is the characteristic function for $[0, c]$, and we have restricted our attention to the smaller domain $\Pi_{T}^{\mathrm{R}}$. Finally, we have used the fact that $\gamma(x) \equiv \gamma_{\mathrm{R}}$ for $x \in\left(0, x_{\mathrm{R}}\right)$.

Next, note that for $c \leq u_{\mathrm{c}}$ in $(4.17)$, the term $(A(u)-A(c))_{-}$vanishes. This is easy to see by considering the two possible cases $u>c$, and $u \leq c$. In the first case, $A(u)-A(c) \geq 0$, since $A(\cdot)$ is nondecreasing, and in the second case $A(u)-A(c)=0$, since $A(\cdot)$ is constant on $\left[0, u_{\mathrm{c}}\right]$. Also, $\hat{f}(\gamma, u)-\hat{f}(\gamma, c)=f(\gamma, u)-f(\gamma, c)$. Thus, we conclude from (4.17) that

$$
\left(\chi_{l}(w ; c)\left(\hat{f}\left(\gamma_{\mathrm{R}}, u\right)-\hat{f}\left(\gamma_{\mathrm{R}}, c\right)\right)\right)_{x} \in \mathcal{M}\left(\Pi_{T}^{\mathrm{R}}\right) \quad \text { for } c \leq u_{\mathrm{c}} .
$$

In (4.18), we now take $c_{1}:=u_{1}^{*} \wedge u_{\mathrm{c}}, c_{2}:=u_{2}^{*} \wedge u_{\mathrm{c}}, c_{3}:=1 \wedge u_{\mathrm{c}}$, and letting

$$
P_{i}\left(\gamma_{\mathrm{R}}, u\right):=\chi_{l}\left(w ; c_{i}\right)\left(\hat{f}\left(\gamma_{\mathrm{R}}, u\right)-\hat{f}\left(\gamma_{\mathrm{R}}, c_{i}\right)\right), \quad i=1,2,3,
$$

we have $\partial_{x} P_{i} \in \mathcal{M}\left(\Pi_{T}^{\mathrm{R}}\right)$. It is a straightforward exercise to verify that

$$
\mathcal{F}(\boldsymbol{\gamma}, u)=P_{3}\left(\gamma_{\mathrm{R}}, u\right)-2 P_{2}\left(\gamma_{\mathrm{R}}, u\right)+2 P_{1}\left(\gamma_{\mathrm{R}}, u\right)+\hat{f}\left(\boldsymbol{\gamma}_{\mathrm{R}}, c_{3}\right)-2 \hat{f}\left(\boldsymbol{\gamma}_{\mathrm{R}}, c_{2}\right)+2 \hat{f}\left(\boldsymbol{\gamma}_{\mathrm{R}}, c_{1}\right),
$$

from which it follows immediately that $\mathcal{F}(\gamma, u)_{x} \in \mathcal{M}\left(\Pi_{T}^{\mathrm{R}}\right)$.

The proof of the following lemma is a direct application of Lemma 4.3. Its proof follows from the proofs of Lemmas 3.3 and 3.4 of [56].

Lemma 4.4. $A B V_{t}$ entropy solution $u$ and the quantities $\gamma_{1} A(u), \gamma_{1} A(u)_{x}, \gamma_{1}|A(u)-A(c)|_{x}$ admit right and left traces at each jump in $\gamma$.

4.5. Entropy jump conditions and uniqueness of entropy solutions. Our objective in this section is to prove the $L^{1}$ stability of entropy weak solutions, which is stated in Theorem 4.1. If we had in our problem (4.1) the parabolic term $A(u)_{x x}$ instead of $\left(\gamma_{1}(x) A(u)_{x}\right)_{x}$, i.e., if $\gamma_{1} \equiv 1$, Section 2 of [56] would apply verbatim, and we could simply appeal to Theorem 2.1 of that paper. Thus we will follow closely Section 2 of [56]. Since the spatially varying parameter $\gamma_{1}$ plays a key role here, we remind the reader that $\gamma_{1}$ is simply the characteristic function for the interval $\left(x_{\mathrm{L}}, x_{\mathrm{R}}\right)$.

As in [56], it is convenient, and sufficient, to work with limits in the sense of Lebesgue. Specifically, let $W=W(x)$ be any function on $\mathbb{R}$, and fix a point $x_{0} \in \mathbb{R}$. We define Lebesgue type one-sided limits as follows:

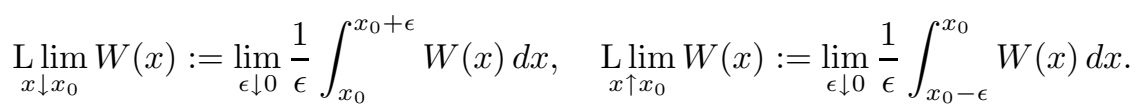

The key fact here (see Lemma 2.1 of [56]) is the following.

Lemma 4.5. Let $W \in L^{\infty}\left(\Pi_{T}\right)$, and fix a point $x_{0} \in \mathbb{R}$. If the right and left traces $t \mapsto W\left(x_{0} \pm, t\right)$ exist in the sense of Definition 4.2, then for a.e. $t \in(0, T)$ they also exist as right and left traces in the sense of Lebesgue points in $L^{1}$ :

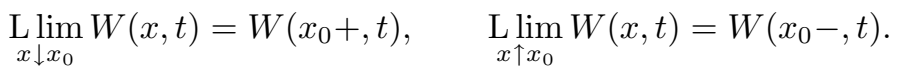

Next, we record the versions of Lemmas 2.2 and 2.3 of [56] that account for the coefficient $\gamma_{1}(x)$ multiplying $A(u)_{x}$. The proofs in [56] are easily modified to deal with $\gamma_{1}$, and are omitted here. 
Lemma 4.6. Let $u$ and $v$ be a pair of $B V_{t}$ entropy weak solutions. Let $F$ be the Kružkov entropy flux defined in (4.12). Fix one of the jumps in $\gamma$ located at $m \in \mathcal{J}$. Then for a.e. $t \in(0, T)$

$$
\begin{aligned}
& \mathrm{L} \lim _{x \downarrow m} F(\gamma(x), u(x, t), v(x, t))=F(\gamma(m+), u(m+, t), v(m+, t)), \\
& \mathrm{L}_{x \uparrow m} F(\gamma(x), u(x, t), v(x, t))=F(\gamma(m-), u(m-, t), v(m-, t)), \\
& \operatorname{Lim}_{x \downarrow m}\left(\gamma_{1}(x)|A(u)-A(v)|_{x}\right)(x, t) \\
& = \begin{cases}\gamma_{1}(m+) \sigma(m+, t)\left(A(u)_{x}(m+, t)-A(v)_{x}(m+, t)\right) & \text { if } A(u(m+, t)) \neq A(v(m+, t)), \\
\gamma_{1}(m+)\left|A(u)_{x}(m+, t)-A(v)_{x}(m+, t)\right| & \text { otherwise, }\end{cases} \\
& \mathrm{L} \lim _{x \uparrow m}\left(\gamma_{1}(x)|A(u)-A(v)|_{x}\right)(x, t) \\
& = \begin{cases}\gamma_{1}(m-) \sigma(m-, t)\left(A(u)_{x}(m-, t)-A(v)_{x}(m-, t)\right) & \text { if } A(u(m-, t)) \neq A(v(m-, t)), \\
-\gamma_{1}(m-)\left|A(u)_{x}(m-, t)-A(v)_{x}(m-, t)\right| & \text { otherwise, }\end{cases}
\end{aligned}
$$

where $\sigma(m+, t):=\operatorname{sgn}(u(m+, t)-v(m+, t))$ and $\sigma(m-, t):=\operatorname{sgn}(u(m-, t)-v(m-, t))$.

Lemma 4.7. Let u be a $B V_{t}$ entropy weak solution. Let $F$ be the Kružkov entropy flux defined in (4.12). Fix one of the jumps in $\gamma$ located at $m \in \mathcal{J}$. For any $c \in \mathbb{R}$, we have for a.e. $t \in(0, T)$.

$$
\begin{aligned}
& \mathrm{L}_{x \downarrow m} F(\gamma(x), u(x, t), c)=F(\gamma(m+), u(m+, t), c), \\
& \mathrm{L}_{x \uparrow m} F(\gamma(x), u(x, t), c)=F(\gamma(m-), u(m-, t), c), \\
& \mathrm{L}_{x \downarrow m}\left(\gamma_{1}(x)|A(u)-A(c)|_{x}\right)(x, t)= \begin{cases}\gamma_{1}(m+) \sigma(m+, t) A(u)_{x}(m+, t) & \text { if } u(m+, t) \neq c, \\
\gamma_{1}(m+)\left|A(u)_{x}(m+, t)\right| & \text { if } u(m+, t)=c,\end{cases} \\
& \mathrm{L}_{x \uparrow m}\left(\gamma_{1}(x)|A(u)-A(c)|_{x}\right)(x, t)= \begin{cases}\gamma_{1}(m-) \sigma(m-, t) A(u)_{x}(m-, t) & \text { if } u(m-, t) \neq c, \\
-\gamma_{1}(m-)\left|A(u)_{x}(m-, t)\right| & \text { if } u(m-, t)=c,\end{cases}
\end{aligned}
$$

where $\sigma(m-, t):=\operatorname{sgn}(u(m-, t)-c)$ and $\sigma(m+, t):=\operatorname{sgn}(u(m+, t)-c)$.

Before continuing, we introduce a notational convention that we hope will simplify the appearance of the formulas that follow. Whenever we are discussing a fixed element $m \in \mathcal{J}$, and the time is fixed at $t \in[0, T]$ where all of the relevant right and left limits exist, we use the notation $u_{ \pm}=u_{ \pm}(t)=u(m \pm, t), \gamma_{ \pm}=\gamma(m \pm)$ and $\left(\gamma_{1} A\left(u_{x}\right)\right)_{ \pm}=\left(\gamma_{1} A(u)_{x}\right)(m \pm, t)$.

We collect in the following lemma several properties of a $B V_{t}$ entropy weak solution near a jump in $\gamma$. The relationship (4.26) is the Rankine-Hugoniot condition for this problem, while (4.28) is an entropy condition. The relationship (4.27) restricts the sign of $A(u)_{x}$ at a jump in $\gamma$.

Remark 4.1. To highlight once again the significance of assumption (D.4), we mention that the proof of (4.27) requires the hypothesis that $A(u)$ is continuous across the jumps in $\gamma$ at $x_{\mathrm{L}}$ and $x_{\mathrm{R}}$. Thus, (D.4) is crucial for the uniqueness of entropy weak solutions of Model 1.

Lemma 4.8. Let $u$ be a $B V_{t}$ entropy weak solution. Fix one of the jumps in $m \in \mathcal{J}$. Then the following relationships hold across the jump for a.e. $t \in(0, T)$ :

$$
\begin{gathered}
f\left(\gamma_{+}, u_{+}\right)-\left(\gamma_{1} A(u)_{x}\right)_{+}=f\left(\gamma_{-}, u_{-}\right)-\left(\gamma_{1} A(u)_{x}\right)_{-}, \\
\operatorname{sgn}\left(u_{+}-u_{-}\right) \operatorname{sgn}\left(\left(A(u)_{x}\right)_{+}\right) \geq 0, \quad \operatorname{sgn}\left(u_{+}-u_{-}\right) \operatorname{sgn}\left(\left(A(u)_{x}\right)_{-}\right) \geq 0,
\end{gathered}
$$

and for $u_{-}(t) \neq u_{+}(t)$ :

$$
\begin{aligned}
& {\left[F\left(\gamma_{+}, u_{+}, c\right)-\operatorname{sgn}\left(u_{+}-c\right)\left(\gamma_{1} A(u)_{x}\right)_{+}\right]-\left[F\left(\gamma_{-}, u_{-}, c\right)-\operatorname{sgn}\left(u_{-}-c\right)\left(\gamma_{1} A(u)_{x}\right)_{-}\right]} \\
& \quad \leq\left|f\left(\gamma_{+}, c\right)-f\left(\gamma_{-}, c\right)\right| \quad \forall c \in \mathbb{R},
\end{aligned}
$$

where $F$ is the Kružkov entropy flux function defined in (4.12). In addition, the appropriate inequality in Table 1 holds for all $c$ between $u_{-}$and $u_{+}$. 


\begin{tabular}{c|cc} 
& $f\left(\gamma_{-}, c\right) \leq f\left(\boldsymbol{\gamma}_{+}, c\right)$ & $f\left(\boldsymbol{\gamma}_{-}, c\right) \geq f\left(\boldsymbol{\gamma}_{+}, c\right)$ \\
\hline$u_{-} \leq c \leq u_{+}$ & $f\left(\boldsymbol{\gamma}_{+}, u_{+}\right)-\left(\gamma_{1} A(u)_{x}\right)_{+} \leq f\left(\boldsymbol{\gamma}_{+}, c\right)$ & $f\left(\boldsymbol{\gamma}_{-}, u_{-}\right)-\left(\gamma_{1} A(u)_{x}\right)_{-} \leq f\left(\boldsymbol{\gamma}_{-}, c\right)$ \\
$u_{+} \leq c \leq u_{-}$ & $f\left(\boldsymbol{\gamma}_{-}, u_{-}\right)-\left(\gamma_{1} A(u)_{x}\right)_{-} \geq f\left(\boldsymbol{\gamma}_{-}, c\right)$ & $f\left(\boldsymbol{\gamma}_{+}, u_{+}\right)-\left(\gamma_{1} A(u)_{x}\right)_{+} \geq f\left(\boldsymbol{\gamma}_{+}, c\right)$
\end{tabular}

TABLE 1. Entropy jump conditions.

Proof. The proofs of these assertions are similar to the proofs of Lemmas 2.4, 2.5, 2.6, and 2.7 of [56]. Since the proof of (4.27) relies on the assumption (D.4), we will review its proof, but will not repeat the proofs of the other assertions. We start by fixing a time $t \in(0, T)$ where all of the relevant right and left spatial (essential) limits exist at $x=m$. We only prove the first inequality in (4.27); the proof of the other inequality is similar. Let us suppress the dependence on $t$ for the remainder of the proof. If $u_{+}=u_{-}$, the inequality is obvious, so assume that $u_{+}>u_{-}$. We must show that $\left(A(u)_{x}\right)_{+} \geq 0$. From

$$
\underset{\varepsilon \downarrow 0}{\operatorname{ess}} \lim u(m+\varepsilon)=: u_{+}>u_{-},
$$

it is clear that $u(m+\varepsilon)>u_{-}$for a.e. sufficiently small $\varepsilon>0$. Next, we apply $A$ to both sides of $u(m+\varepsilon)>u_{-}$. Since $A$ is nondecreasing, we obtain $A(u(m+\varepsilon)) \geq A\left(u_{-}\right)$for a.e. sufficiently small $\varepsilon>0$. If the jump point is at the origin, i.e., $m=0$, then continuity of $A(u)$ follows from assumption (D.1). If $m=x_{\mathrm{L}}$ or $m=x_{\mathrm{R}}$, then assumption (D.4) gives us continuity of $A(u)$. In either case, we have $A\left(u_{-}\right)=A\left(u_{+}\right)$. Thus,

$$
\frac{1}{\varepsilon} \int_{m}^{m+\varepsilon} A(u)_{x} d x=\frac{1}{\varepsilon}\left(A(u(m+\varepsilon))-A\left(u_{+}\right)\right) \geq 0,
$$

for a.e. sufficiently small $\varepsilon>0$. Letting $\varepsilon \downarrow 0$ (along a subsequence for which (4.29) holds) yields $\left(A(u)_{x}\right)_{+} \geq 0$. The proof is completed by showing via a similar argument that if $u_{+}<u_{-}$, then $\left(A(u)_{x}\right)_{+} \leq 0$.

Remark 4.2. Since $A$ is nondecreasing, we can write the entropy condition (4.28) in the alternative form

$$
\Phi(\boldsymbol{\gamma}, u, c)_{+}-\Phi(\gamma, u, c)_{-} \leq\left|f\left(\gamma_{+}, c\right)-f\left(\gamma_{-}, c\right)\right| \quad \text { for all } c \in \mathbb{R},
$$

where $\Phi(\gamma, u, c):=F(\gamma, u, c)-\gamma_{1}|A(u)-A(c)|_{x}$. Note that the entropy jump condition (4.28) is the same as the one stated in Lemma 2.6 of [56], with the exception that $\gamma_{1}=\gamma_{1}(x)$ is not present in [56]. Similarly, this is the only difference between Table 1 of [56] and our Table 1.

The next lemma is basically Lemma 2.8 of [56], adapted to the present setup.

Lemma 4.9. Let $u$ be a $B V_{t}$ entropy weak solution. Fix the jump in $\gamma$ located at $m=0$, and a time $t \in[0, T]$ where all of the relevant right and left limits exist. If $u_{-} \neq u_{+}$, then $A^{\prime}(w)=0$ for $w$ between $u_{-}$and $u_{+}$, and thus $A(\cdot)$ is constant on the interval connecting $u_{-}$to $u_{+}$, that is,

$$
A(w)=A\left(u_{-}\right)=A\left(u_{+}\right) \quad \text { for } w \text { between } u_{-} \text {and } u_{+} .
$$

Taken together, Lemma 4.9 and assumption (D.4) guarantee continuity of $x \mapsto A(u(x, t))$ for a.e. $t \in[0, T]$ at each of the jumps $m \in\left\{x_{\mathrm{L}}, 0, x_{\mathrm{R}}\right\}$. Using this fact, along with the relationships in Table 1, it is possible to prove the following lemma, whose proof we omit, since it is essentially the same as the proof of Lemma 2.9 of [56].

Lemma 4.10. Let $u$ and $v$ be a pair of $B V_{t}$ entropy weak solutions. Fix a jump in $\gamma$ located at $m \in \mathcal{J}$ and a time $t \in(0, T)$ where all of the relevant right and left traces exist. Assume that $u_{-}>v_{-}, \quad u_{+}<v_{+}$. If $u_{+} \leq u_{-}$, then

$$
\begin{aligned}
v_{-} \in & {\left[u_{+}, u_{-}\right), f\left(\gamma_{+}, v_{-}\right) \geq f\left(\gamma_{-}, v_{-}\right) } \\
& \Longrightarrow f\left(\gamma_{-}, v_{-}\right)-\left(\gamma_{1} A(v)_{x}\right)_{-} \leq f\left(\gamma_{-}, u_{-}\right)-\left(\gamma_{1} A(u)_{x}\right)_{-}, \\
v_{+} \in & \left(u_{+}, u_{-}\right], \quad f\left(\gamma_{-}, v_{+}\right) \geq f\left(\gamma_{+}, v_{+}\right) \\
& \Longrightarrow f\left(\gamma_{+}, v_{+}\right)-\left(\gamma_{1} A(v)_{x}\right)_{+} \leq f\left(\gamma_{+}, u_{+}\right)-\left(\gamma_{1} A(u)_{x}\right)_{+} .
\end{aligned}
$$


If $v_{-} \leq v_{+}$, then

$$
\begin{aligned}
& u_{-} \in\left(v_{-}, v_{+}\right], f\left(\gamma_{+}, u_{-}\right) \leq f\left(\gamma_{-}, u_{-}\right) \\
& \Longrightarrow f\left(\gamma_{-}, v_{-}\right)-\left(\gamma_{1} A(v)_{x}\right)_{-} \leq f\left(\gamma_{-}, u_{-}\right)-\left(\gamma_{1} A(u)_{x}\right)_{-}, \\
& u_{+} \in {\left[v_{-}, v_{+}\right), f\left(\gamma_{+}, u_{+}\right) \geq f\left(\gamma_{-}, u_{+}\right) } \\
& \quad \Longrightarrow f\left(\gamma_{+}, v_{+}\right)-\left(\gamma_{1} A(v)_{x}\right)_{+} \leq f\left(\gamma_{+}, u_{+}\right)-\left(\gamma_{1} A(u)_{x}\right)_{+} .
\end{aligned}
$$

Before proceeding to our main uniqueness theorem, let us recall the so-called crossing condition that we introduced in [56].

Definition 4.3 (Crossing condition). For any jump in $\gamma$ with associated left and right limits $\left(\gamma_{-}, \gamma_{+}\right)$, we say that the crossing condition holds, if for any states $u$ and $v$,

$$
f\left(\boldsymbol{\gamma}_{+}, u\right)-f\left(\boldsymbol{\gamma}_{-}, u\right)<0<f\left(\boldsymbol{\gamma}_{+}, v\right)-f\left(\boldsymbol{\gamma}_{-}, v\right) \text { implies } u<v .
$$

The geometric interpretation of this condition is that if the graphs of $u \mapsto f\left(\gamma_{-}, u\right)$ and $u \mapsto$ $f\left(\gamma_{+}, u\right)$ cross, then there can be at most one crossing, say at $u=u_{\chi}$, and in this case the graph of $f\left(\gamma_{-}, u\right)$ lies above (below) the graph of $f\left(\gamma_{+}, u\right)$ for $u<u_{\chi}\left(u>u_{\chi}\right)$. The crossing condition is satisfied automatically if there is no crossing. Figure $2(\mathrm{~d})$ shows an example of a flux crossing that satisfies the crossing condition, with crossing point $u_{\chi}=u_{\mathrm{F}}$. A motivation for the crossing condition in the present context is given by the following lemma, whose elementary proof is provided in [25]. See also Figure 2.

Lemma 4.11. With our assumptions on $b, q_{\mathrm{L}}$ and $q_{\mathrm{R}}$, the crossing condition is satisfied at each jump $m \in \mathcal{J}$. Specifically, there are no flux crossings associated with the jumps $x=x_{\mathrm{L}}, x=x_{\mathrm{R}}$. There may be a single crossing at the jump $x=0$, but it satisfies the crossing condition.

Satisfaction of the crossing condition at the nontrivial crossing at $x=0$ may be traced to the fact that a source is located there, and thus the flow diverges from the origin. This is most easily understood by ignoring for a moment the batch flux $b(u)$ and the parabolic term. It is easy to check that these terms do not affect the crossing relationship at $x=0$. Then, if we use $\delta(x)$ to denote the delta function, our model simplifies to

$$
u_{t}+q(x) u_{x}=\left(q_{\mathrm{R}}-q_{\mathrm{L}}\right) u_{\mathrm{F}} \delta(x), \quad q(x)= \begin{cases}q_{\mathrm{L}} & \text { for } x<0, \\ q_{\mathrm{R}} & \text { for } x>0 .\end{cases}
$$

Since $q_{\mathrm{L}}<0<q_{\mathrm{R}}$, we have diverging flows, balanced by a source term on the right side. Notice that in this case the flux curves are the straight lines $u \mapsto q_{\mathrm{L}}\left(u-u_{\mathrm{F}}\right)$ and $u \mapsto q_{\mathrm{R}}\left(u-u_{\mathrm{F}}\right)$, and the crossing condition is satisfied. On the other hand, if we had $q_{\mathrm{L}}>0>q_{\mathrm{R}}$, then our simplified model would result in converging flows, balanced by a sink term, and the crossing condition would be violated. Thus, from a physical point of view, our assumption that the crossing condition is satisfied is a natural one, and follows directly from the fact that the clarifier thickener model has a source term (as opposed to a sink term).

If any of the jumps in $\gamma$ violated the crossing condition, our definition of entropy solution would not be strong enough to rule out so-called "expansion shocks" (see [56] for a detailed explanation), and our uniqueness theory would break down. It turns out that additional entropy conditions are required when the crossing condition is not satisfied; we defer further discussion of this issue to a future paper since the crossing condition is satisfied in the present context.

We are finally able to prove our main uniqueness theorem.

Theorem 4.1 ( $L^{1}$ stability and uniqueness). Let $v$ and $u$ be two $B V_{t}$ entropy weak solutions to the initial value problem (4.1). For a.e. $t \in(0, T)$,

$$
\|v(\cdot, t)-u(\cdot, t)\|_{L^{1}(\mathbb{R})} \leq\left\|v_{0}-u_{0}\right\|_{L^{1}(\mathbb{R})} .
$$

Proof. For $B V_{t}$ entropy weak solutions $u$ and $v$, a "doubling of variables" argument appearing in Appendix A of [56] yields

$$
-\iint_{\Pi_{T}}\left(|v-u| \varphi_{t}+F(\gamma(x), v, u) \varphi_{x}+\left|\gamma_{1} A(v)-\gamma_{1} A(u)\right| \varphi_{x x}\right) d t d x \leq 0
$$


for any $0 \leq \varphi \in \mathcal{D}\left(\Pi_{T} \backslash \mathcal{J}\right)$. Next, via a limiting argument (see the proof of Theorem 2.1 of [56]) we extend this inequality to the larger class of test functions which do not vanish near $x \in \mathcal{J}$. Specifically, we obtain for any $0 \leq \phi \in \mathcal{D}\left(\Pi_{T}\right)$

$$
\begin{gathered}
-\iint_{\Pi_{T}}\left(|v-u| \phi_{t}+F(\gamma(x), v, u) \phi_{x}-\left|\gamma_{1} A(v)-\gamma_{1} A(u)\right| \phi_{x x}\right) d t d x \\
\leq \sum_{m \in \mathcal{J}} \int_{0}^{T}\left[F(\gamma(x), v, u)-\left|\gamma_{1} A(v)-\gamma_{1} A(u)\right|_{x}\right]_{x=m-}^{x=m+} \phi(m, t) d t
\end{gathered}
$$

where the notation indicates limits from the right and left at $x=m$.

We wish to show that each term in the sum on the right side of (4.37) is nonpositive. If we fix a jump point $m \in \mathcal{J}$, then the contribution to this sum from the jump point $m$ is given by

$$
R:=\Phi(\gamma(m+), v(m+, t), u(m+, t))-\Phi(\gamma(m-), v(m-, t), u(m-, t)) .
$$

Here $\Phi$ is defined in Remark 4.2, and appears in the entropy condition (4.30). Our goal is now to show that $R$ is nonpositive. Let us fix a time $t \in(0, T)$ where all of the relevant essential right and left limits exist. If $m=0$, then since $\gamma_{1}=1$ on the interval $\left(x_{\mathrm{L}}, x_{\mathrm{R}}\right)$ containing $x=0$, $R \leq 0$ is immediate by repeating the relevant portion (the 7 cases) of the proof of Theorem 2.1 of [56]. (Note that in [56] we used the symbol $S$ for the quantity known as $R$ here.) We will not reproduce that proof here, but we emphasize that this $(m=0)$ is the only case where a nontrivial flux crossing occurs, and thus we rely on the fact that the crossing condition is satisfied.

If $x=x_{\mathrm{L}}$ or $x=x_{\mathrm{R}}$, then because of the jump in $\gamma_{1}$, we cannot appeal directly to [56], which did not address the case of spatially varying parabolic term. We will focus on the case $x=x_{\mathrm{R}}$, and omit the similar case $x=x_{\mathrm{L}}$. The approach is to verify that $R \leq 0$ in each of the 7 cases identified in [56]. The assumptions on $b(u)$ ensure that at $x=x_{\mathrm{R}}$, we will always have $f\left(\gamma_{-}, u\right) \geq f\left(\gamma_{+}, u\right)$. In particular, there are no flux crossings, which simplifies the proofs of Cases 6 and 7 .

Case $1\left(v_{-}=u_{-}, v_{+}=u_{+}\right)$. Then $F\left(\gamma_{+}, v_{+}, u_{+}\right)=0$ and $F\left(\gamma_{-}, v_{-}, u_{-}\right)=0$, and by Lemma 4.6, and the fact that $\gamma_{1}\left(x_{\mathrm{R}}+\right)=0, R$ reduces to

$$
R=-\left|\left(\gamma_{1} A(u)_{x}\right)_{-}-\left(\gamma_{1} A(v)_{x}\right)_{-}\right| \leq 0 .
$$

Case $2\left(v_{-}=u_{-}, u_{+} \neq v_{+}\right)$. Assume that $v_{+}>u_{+}$. In this case

$$
R=f\left(\gamma_{+}, v_{+}\right)-f\left(\gamma_{+}, u_{+}\right)-\left(\gamma_{1} A(v)_{x}\right)_{+}+\left(\gamma_{1} A(u)_{x}\right)_{+}-\left|\left(\gamma_{1} A(v)_{x}\right)_{-}-\left(\gamma_{1} A(u)_{x}\right)_{-}\right| \text {, }
$$

where we have used the equality $f\left(\gamma_{-}, v_{-}\right)=f\left(\gamma_{-}, u_{-}\right)$. Via the Rankine-Hugoniot condition, and another application of $f\left(\gamma_{-}, u_{-}\right)=f\left(\gamma_{-}, v_{-}\right)$, we get

$$
f\left(\gamma_{+}, v_{+}\right)-f\left(\gamma_{+}, u_{+}\right)=-\left(\gamma_{1} A(v)_{x}\right)_{-}+\left(\gamma_{1} A(u)_{x}\right)_{-} .
$$

We have again used the fact that $\gamma_{1}\left(x_{\mathrm{R}}+\right)=0$. Substituting this into (4.39) gives

$$
R=-\left|\left(\gamma_{1} A(v)_{x}\right)_{-}-\left(\gamma_{1} A(u)_{x}\right)_{-}\right| \leq 0 .
$$

The situation where $v_{+}<u_{+}$is handled similarly.

Case $3\left(v_{+}=u_{+}, u_{-} \neq v_{-}\right)$. The proof of this case is similar to that of Case 2 , and therefore omitted.

Case $4\left(u_{-}<v_{-}, u_{+}<v_{+}\right)$. In this case, using $\gamma_{1}\left(x_{\mathrm{R}}+\right)=0$, we obtain from (4.38)

$$
R=\left(f\left(\gamma_{+}, v_{+}\right)-f\left(\gamma_{+}, u_{+}\right)\right)-\left[f\left(\gamma_{-}, v_{-}\right)-f\left(\gamma_{+}, u_{+}\right)-\left(\gamma_{1} A(v)_{x}\right)_{-}+\left(\gamma_{1} A(u)_{x}\right)_{-}\right],
$$

which equals zero, by the Rankine-Hugoniot condition (4.26).

Case $5\left(u_{-}>v_{-}, u_{+}>v_{+}\right)$. As in the preceding case, $R=0$ due to a similar calculation.

Case $6\left(u_{-}>v_{-}, u_{+}<v_{+}\right)$. In this case, (4.38) becomes

$$
\begin{aligned}
R & =f\left(\gamma_{-}, v_{-}\right)+f\left(\gamma_{+}, v_{+}\right)-f\left(\gamma_{-}, u_{-}\right)-f\left(\gamma_{+}, u_{+}\right)-\left(\gamma_{1} A(v)_{x}\right)_{-}+\left(\gamma_{1} A(u)_{x}\right)_{-} \\
& =2 f\left(\gamma_{+}, v_{+}\right)-2 f\left(\gamma_{+}, u_{+}\right) \\
& =2\left[f\left(\gamma_{-}, v_{-}\right)-\left(\gamma_{1} A(v)_{x}\right)_{-}\right]-2\left[f\left(\gamma_{-}, u_{-}\right)-\left(\gamma_{1} A(u)_{x}\right)_{-}\right],
\end{aligned}
$$

where (4.41) and (4.42) follow from the Rankine-Hugoniot condition (4.26). In (4.41) we have used again the fact that $\gamma_{1}\left(x_{\mathrm{R}}+\right)=0$. It follows from the assumption $u_{-}>v_{-}, u_{+}<v_{+}$that 
$u_{+}<v_{+} \leq u_{-}$or $v_{-}<u_{-} \leq v_{+}$must hold. Take the case where $u_{+}<v_{+} \leq u_{-}$. Recalling that at $m=x_{\mathrm{R}}$ we always have $f\left(\gamma_{-}, \cdot\right) \geq f\left(\gamma_{+}, \cdot\right)$, we can apply (4.32) of Lemma 4.10, giving us $f\left(\gamma_{+}, v_{+}\right) \leq f\left(\gamma_{+}, u_{+}\right)$. With (4.41) in mind, we see that that $R \leq 0$. In the case where $v_{-}<u_{-} \leq v_{+},(4.33)$ of Lemma 4.10 yields $f\left(\gamma_{-}, v_{-}\right)-\left(\gamma_{1} A(v)_{x}\right)_{-} \leq f\left(\gamma_{-}, u_{-}\right)-\left(\gamma_{1} A(u)_{x}\right)_{-}$, again implying that $R \leq 0$, this time using (4.42)

Case $7\left(u_{-}<v_{-}, u_{+}>v_{+}\right)$. The proof is identical to that of Case 6 ; we switch the roles of $u$ and $v$, and use the version of Lemma 4.10 that results by also switching the roles of $u$ and $v$.

We have established that for any $0 \leq \phi \in \mathcal{D}\left(\Pi_{T}\right)$

$$
-\iint_{\Pi_{T}}\left(|v-u| \phi_{t}+F(\gamma(x), v, u) \phi_{x}-\left|\gamma_{1} A(v)-\gamma_{1} A(u)\right| \phi_{x x}\right) d t d x \leq 0 .
$$

The proof is concluded via a standard test function argument which is outlined in the proof of Theorem 2.1 of [56].

4.6. Convergence of the numerical approximations. In the sequel, let us denote by $\Delta$ the pair $\Delta:=(\Delta t, \Delta x)$. Our purpose in this section is to prove convergence (along a subsequence) of the numerical approximations as $\Delta \downarrow 0$, i.e., as $\Delta t, \Delta x \rightarrow 0$ with $\Delta t, \Delta x>0$. For the sake of simplicity, we will concentrate on the explicit version of the algorithm.

Let $\left(\gamma^{\Delta}, u^{\Delta}\right)$ be the finite difference approximation defined in (3.3). A significant part of the convergence analysis consists of establishing a spatial total variation estimate for the approximate solution $u^{\Delta}$, measured with respect to a particular transformed variable. More precisely, we prove that $u^{\Delta}$ converges (along a subsequence) to a weak solution by introducing a singular mapping $\Psi:(\gamma, u) \mapsto(\gamma, z)$ such that strong compactness of $z^{\Delta}=\Psi\left(\gamma^{\Delta}, u^{\Delta}\right)$ can be obtained. As always in problems involving resonance phenomena, one should measure the space translates with respect to a nonlinear transformation; as already mentioned in the introduction, there is generally no spatial total variation bound for the conserved variable $u$ itself. The singular mapping approach has been used for many years in the purely hyperbolic setting, starting with the paper [76].

On the other hand, the construction of a suitable singular mapping $\Psi$ for second order equations is more recent, and was done first in [55]. The idea is to construct a singular mapping that includes a contribution both from the convective flux and the diffusion function. We first prove compactness for the two parts of the singular mapping separately. We then combine the two portions to recover the original singular mapping and conclude that since the mapping is strictly increasing as a function of the conserved variable $u$, convergence of the transformed variable implies convergence of $u$.

Since we are applying the scheme described in Section 3 to Model 1 (constant cross section), we can simplify the analysis by taking $S_{j} \equiv 1$, and then $\lambda_{j}=: \lambda=\Delta t / \Delta x$. To simplify the notation a little further, let $\mu=\lambda / \Delta x, h_{j+1 / 2}^{n}=h\left(\gamma_{j+1 / 2}, U_{j+1}^{n}, U_{j}^{n}\right), \gamma_{1 j+1 / 2}=s_{j+1 / 2}$, and $A_{j}^{n}=A\left(U_{j}^{n}\right)$. The marching formula (3.1) then takes the form

$$
U_{j}^{n+1}=U_{j}^{n}-\lambda \Delta_{+} h_{j-1 / 2}^{n}+\mu \Delta_{+}\left(s_{j-1 / 2} \Delta_{-} A_{j}^{n}\right)
$$

The EO numerical flux is consistent with the actual flux, i.e., $h(\gamma, u, u)=f(\boldsymbol{\gamma}, u)$. In addition, for fixed $\boldsymbol{\gamma}, h(\gamma, v, u)$ is a two-point monotone flux, meaning that it is nonincreasing with respect to $v$, and nondecreasing with respect to $u$. Due to the regularity assumptions on $f$, the numerical flux $h$ is Lipschitz continuous with respect to each of its arguments, and in fact satisfies

$$
f_{u}^{-}(\boldsymbol{\gamma}, v)=h_{v}(\boldsymbol{\gamma}, v, u) \leq 0 \leq h_{u}(\boldsymbol{\gamma}, v, u)=f_{u}^{+}(\boldsymbol{\gamma}, u),
$$

where $f_{u}^{-}(\gamma, u):=\min \left\{0, f_{u}(\gamma, u)\right\}$ and $f_{u}^{+}(\gamma, u):=\max \left\{0, f_{u}(\gamma, u)\right\}$ denote the negative and positive parts of $f_{u}$. Thus, whenever the flux $u \mapsto f(\gamma, u)$ is $C^{1}$, the numerical flux is also $C^{1}$ as a function of the conserved variables $u$ and $v$. The following bound is easily checked:

$$
\|h\|:=\max \{|h(\gamma, v, u)| \mid \gamma \in \mathcal{G}, v, u \in[0,1]\} \leq\|f\|+\frac{1}{2}\left\|f_{u}\right\| .
$$

From formula (4.45) it is clear that $\left\|f_{u}\right\|$ is a Lipschitz constant for the numerical flux $h$ with respect to the conserved variables $u$ and $v$. 
We assume that the discretization parameters $(\Delta x, \Delta t)$ are chosen so that the following CFL (Courant-Friedrichs-Lewy [35]) condition holds:

$$
\lambda\left(\max \left\{-q_{\mathrm{L}}, q_{\mathrm{R}}\right\}+\max _{u \in[0,1]}\left|b^{\prime}(u)\right|\right)+\mu \max _{u \in[0,1]} A^{\prime}(u) \leq 1 / 2 .
$$

In our case, the stability analysis relies very much on the monotonicity property of the scheme, where we recall that a finite difference scheme such as (4.44) is monotone [36] if

$$
U_{j}^{n} \leq V_{j}^{n} \quad \forall j \quad \Longrightarrow \quad U_{j}^{n+1} \leq V_{j}^{n+1} \quad \forall j .
$$

The following lemma and its proof illustrate how the CFL condition (4.46) is derived from the requirement that our scheme $(4.44)$ be monotone.

Lemma 4.12. If the initial data $\left\{U_{j}^{0}\right\}_{j \in \mathbb{Z}}$ lies in the interval $[0,1]$, and the CFL condition (4.46) is satisfied, then the solution $\left\{U_{j}^{n}\right\}_{j \in \mathbb{Z}}$ computed by the explicit scheme (4.44) also belongs to the interval $[0,1]$ for each $n \geq 0$. Moreover, the difference scheme (4.44) remains monotone at each time level $n \geq 0$.

Proof. Let us first rewrite (4.44) as $U_{j}^{n+1}=G_{j}\left(U_{j+1}^{n}, U_{j}^{n}, U_{j-1}^{n}, \gamma_{j+1 / 2}, \gamma_{j-1 / 2}\right)$ for $j \in \mathbb{Z}$. Then the scheme is monotone, i.e., satisfies (4.47), if

$$
\partial U_{j}^{n+1} / \partial U_{j+1}^{n} \geq 0, \quad \partial U_{j}^{n+1} / \partial U_{j}^{n} \geq 0, \quad \partial U_{j}^{n+1} / \partial U_{j-1}^{n} \geq 0, \quad j \in \mathbb{Z} .
$$

However, in our case we have for $j \in \mathbb{Z}$

$$
\begin{aligned}
\partial U_{j}^{n+1} / \partial U_{j+1}^{n}= & -\lambda f_{u}^{-}\left(\gamma_{j+1 / 2}, U_{j+1}^{n}\right)+\mu \gamma_{1_{j+1 / 2}} A^{\prime}\left(U_{j+1}^{n}\right), \\
\partial U_{j}^{n+1} / \partial U_{j-1}^{n}= & \lambda f_{u}^{+}\left(\gamma_{j-1 / 2}, U_{j-1}^{n}\right)+\mu \gamma_{1-1 / 2} A^{\prime}\left(U_{j-1}^{n}\right), \\
\partial U_{j}^{n+1} / \partial U_{j}^{n}= & 1+\lambda f_{u}^{-}\left(\gamma_{j+1 / 2}, U_{j}^{n}\right)-\lambda f_{u}^{+}\left(\gamma_{j-1 / 2}, U_{j}^{n}\right) \\
& -\mu\left(\gamma_{1 j-1 / 2}+\gamma_{1 j+1 / 2}\right) A^{\prime}\left(U_{j}^{n}\right) .
\end{aligned}
$$

Since $f_{u}^{-} \leq 0, f_{u}^{+} \geq 0$ and $A^{\prime}(u) \geq 0$ by definition, we see that the right-hand sides of (4.49) and (4.50) are always nonnegative. If $U_{j}^{n} \in[0,1]$, then it is easy to deduce from (4.51) and from $\gamma_{1} \in[0,1]$ that also $\partial U_{j}^{n+1} / \partial U_{j}^{n} \geq 0$ if the CFL condition (4.46) is satisfied. Precisely speaking, $U_{j}^{n} \in[0,1]$ and the CFL condition (4.46) ensure that the scheme is monotone at time $t_{n}$.

It remains to prove that if we have $U_{j}^{n} \in[0,1]$, then the quantities $U_{j}^{n+1}$ calculated by the scheme also satisfy $U_{j}^{n+1} \in[0,1]$ for $j \in \mathbb{Z}$. To this end, we now apply the scheme (4.44) to the initial data $V_{j}^{0} \equiv 0$. The parabolic terms vanish, since the data is constant, and at time level 1 we get $V_{j}^{1}=V_{j}^{0}-\lambda \Delta_{-} h\left(\gamma_{j+1 / 2}, V_{j+1}^{0}, V_{j}^{0}\right)$. Since $b(u)=0$ for $u=0$ and $u=1$, it is easy to check that $V_{0}^{1}=\lambda\left(q_{\mathrm{R}}-q_{\mathrm{L}}\right) u_{\mathrm{F}}$ and $V_{j}^{1}=0$ for $j \neq 0$. The CFL condition implies $0 \leq \lambda\left(-q_{\mathrm{L}}\right) \leq 1 / 2$ and $0 \leq \lambda q_{\mathrm{R}} \leq 1 / 2$, which yields $0 \leq \lambda\left(q_{\mathrm{R}}-q_{\mathrm{L}}\right) \leq 1$, and thus $V_{j}^{1} \in[0,1]$.

Next, we apply the scheme (4.44) to the initial data $W_{j}^{0} \equiv 1$, yielding at time level $1 W_{j}^{1}=$ $W_{j}^{0}-\lambda \Delta_{-} h\left(\gamma_{j+1 / 2}, W_{j+1}^{0}, W_{j}^{0}\right)$. This time we find that $W_{0}^{1}=1-\lambda\left(q_{\mathrm{R}}-q_{\mathrm{L}}\right)\left(1-u_{\mathrm{F}}\right)$ and $W_{j}^{1}=1$ for $j \neq 0$. The CFL condition again guarantees that $W_{j}^{1} \in[0,1]$. Thus, $0 \leq V_{j}^{1}, W_{j}^{1} \leq 1$, the CFL condition remains valid for $\left\{V_{j}^{1}\right\}_{j \in \mathbb{Z}}$ and $\left\{W_{j}^{1}\right\}_{j \in \mathbb{Z}}$, and monotonicity implies that $V_{j}^{1} \leq U_{j}^{1} \leq W_{j}^{1}$. Continuing inductively, we see that $0 \leq V_{j}^{n} \leq U_{j}^{n} \leq W_{j}^{n} \leq 1$, the CFL condition remains satisfied at each successive time step, and we continue to have monotonicity for each $n \geq 0$.

When sending $\Delta \downarrow 0$, as we will do in the analysis of the explicit scheme (3.1), the ratio $\mu=\lambda / \Delta x=\Delta t / \Delta x^{2}$ will be kept constant, which means that $\lambda=\mu \Delta x$ is variable with $\lambda \rightarrow 0$ as $\Delta \downarrow 0$.

The CFL condition for the semi-implicit scheme (3.4), which we do not analyze here but use for some of the numerical examples, is

$$
\lambda\left(\max \left\{-q_{\mathrm{L}}, q_{\mathrm{R}}\right\}+\max _{u \in[0,1]}\left|b^{\prime}(u)\right|\right) \leq 1 / 2 .
$$

Consequently, the semi-implicit scheme behaves stably for $\Delta \downarrow 0$ if we fix $\lambda=\Delta t / \Delta x$ such that (4.52) is satisfied. The semi-implicit scheme (3.4) allows a faster computation than the explicit scheme (3.1), since $\Delta t$ needs to be chosen proportional to $\Delta x$, not $\Delta x^{2}$ (as for the explicit scheme). 
Again, the CFL condition (4.52) accrues from the requirement that the scheme be monotone, as we shall see in the following version of Lemma 4.12 for the semi-implicit scheme (3.4). This lemma can be considered as a motivation for the CFL condition (4.52).

Lemma 4.13. If the initial data $\left\{U_{j}^{0}\right\}_{j \in \mathbb{Z}}$ lies in the interval $[0,1]$, and the CFL condition (4.52) is satisfied, then the solution $\left\{U_{j}^{n}\right\}_{j \in \mathbb{Z}}$ computed by the semi-implicit scheme (3.4) also belongs to the interval $[0,1]$ for each $n \geq 0$. Moreover, the difference scheme (3.4) remains monotone at each time level $n \geq 0$.

Proof. Let $V^{n}:=\left\{V_{j}^{n}\right\}_{j \in \mathbb{Z}}$ and $W^{n}:=\left\{W_{j}^{n}\right\}_{j \in \mathbb{Z}}$ satisfy $V_{j}^{n}, W_{j}^{n} \in[0,1]$ for all $j \in \mathbb{Z}$. If we compute $V^{n+1}$ and $W^{n+1}$ using the implicit scheme, then with the help of (4.45) we can write their difference as

$$
\begin{aligned}
W_{j}^{n+1}-V_{j}^{n+1}= & W_{j}^{n}-V_{j}^{n}+\alpha_{j+1 / 2}\left(W_{j+1}^{n}-V_{j+1}^{n}\right)-\beta_{j+1 / 2}\left(W_{j}^{n}-V_{j}^{n}\right) \\
& -\alpha_{j-1 / 2}\left(W_{j}^{n}-V_{j}^{n}\right)+\beta_{j-1 / 2}\left(W_{j-1}^{n}-V_{j-1}^{n}\right) \\
& +s_{j+1 / 2} \theta_{j+1}\left(W_{j+1}^{n+1}-V_{j+1}^{n+1}\right)-\left(s_{j+1 / 2}+s_{j-1 / 2}\right) \theta_{j}\left(W_{j}^{n+1}-V_{j}^{n+1}\right) \\
& +s_{j-1 / 2} \theta_{j-1}\left(W_{j-1}^{n+1}-V_{j-1}^{n+1}\right),
\end{aligned}
$$

where we define for $j \in \mathbb{Z}$

$$
\begin{aligned}
\alpha_{j+1 / 2} & :=-\lambda \int_{0}^{1} f_{u}^{-}\left(\gamma_{j+1 / 2}, V_{j+1}^{n}+\phi\left(W_{j+1}^{n}-V_{j+1}^{n}\right)\right) d \phi \geq 0, \\
\beta_{j+1 / 2} & :=\lambda \int_{0}^{1} f_{u}^{+}\left(\gamma_{j+1 / 2}, V_{j}^{n}+\phi\left(W_{j}^{n}-V_{j}^{n}\right)\right) d \phi \geq 0,
\end{aligned}
$$

and

$$
\theta_{j}:=\mu \frac{A\left(W_{j}^{n+1}\right)-A\left(V_{j}^{n+1}\right)}{W_{j}^{n+1}-V_{j}^{n+1}} \geq 0 .
$$

Let us abbreviate $D_{j}^{n}:=W_{j}^{n}-V_{j}^{n}$, and rearrange (4.53) into the form

$$
\begin{aligned}
\left(1+\left(s_{j+1 / 2}+s_{j-1 / 2}\right) \theta_{j}\right) D_{j}^{n+1}= & \left(1-\beta_{j+1 / 2}-\alpha_{j-1 / 2}\right) D_{j}^{n}+\alpha_{j+1 / 2} D_{j+1}^{n} \\
& +\beta_{j-1 / 2} D_{j-1}^{n}+s_{j+1 / 2} \theta_{j+1} D_{j+1}^{n+1}+s_{j-1 / 2} \theta_{j-1} D_{j-1}^{n+1} .
\end{aligned}
$$

Thanks to the CFL condition (4.52), we have $1-\beta_{j+1 / 2}-\alpha_{j-1 / 2} \geq 0$. Thus all of the coefficients appearing in (4.56) are nonnegative, and taking absolute values results in

$$
\begin{aligned}
\left(1+\left(s_{j+1 / 2}+s_{j-1 / 2}\right) \theta_{j}\right)\left|D_{j}^{n+1}\right| \leq & \left(1-\beta_{j+1 / 2}-\alpha_{j-1 / 2}\right)\left|D_{j}^{n}\right| \\
& +\alpha_{j+1 / 2}\left|D_{j+1}^{n}\right|+\beta_{j-1 / 2}\left|D_{j-1}^{n}\right| \\
& +s_{j+1 / 2} \theta_{j+1}\left|D_{j+1}^{n+1}\right|+s_{j-1 / 2} \theta_{j-1}\left|D_{j-1}^{n+1}\right| .
\end{aligned}
$$

Summing (4.57) over $j \in \mathbb{Z}$, cancelling wherever possible, and recalling the definition of $D_{j}^{n}$, we find that

$$
\sum_{j \in \mathbb{Z}}\left|W_{j}^{n+1}-V_{j}^{n+1}\right| \leq \sum_{j \in \mathbb{Z}}\left|W_{j}^{n}-V_{j}^{n}\right|,
$$

indicating that the semi-implicit scheme is $L^{1}$-contractive on data that is constrained to the interval $[0,1]$. It now follows from the Crandall-Tartar lemma [37] that the scheme is also monotone (on data that is constrained to the interval $[0,1])$.

We still must show that the solution remains in $[0,1]$. First, observe that the $L^{1}$-contraction property (4.58) implies that the solution to the implicit scheme is unique. Referring back to the portion of the proof of Lemma 4.12 where we used the specific data $V_{j}^{0} \equiv 0$, and $W_{j}^{0} \equiv 1$, we see that the solutions $V_{j}^{1}$ and $W_{j}^{1}$ are also (the unique) solutions to the implicit scheme at time level 1. With these observations in mind, it is clear that the relevant portion of the proof of Lemma 4.12 also shows invariance of the interval $[0,1]$ for the semi-implicit scheme.

We now continue our analysis of the explicit scheme (3.1). 
Lemma 4.14. Our numerical approximation satisfies the following discrete time continuity estimate (which is uniform in $n$ and $\Delta$ ):

$$
\Delta x \sum_{j \in \mathbb{Z}}\left|U_{j}^{n+1}-U_{j}^{n}\right| \leq \Delta x \sum_{j \in \mathbb{Z}}\left|U_{j}^{1}-U_{j}^{0}\right| \leq C \Delta t,
$$

as well as a uniform (in $n$ and $\Delta$ ) $L^{1}$ bound:

$$
\left\|u^{\Delta}\left(\cdot, t^{n}\right)\right\|_{L^{1}(\mathbb{R})} \leq C T .
$$

Proof. The proof of Lemma 3.3 of [55] is almost entirely applicable to (4.59), the only possible complication arising when we have to bound the quantity

$$
\sum_{j \in \mathbb{Z}}\left|\Delta_{-} \frac{1}{\Delta x} s_{j+1 / 2} \Delta_{+} A\left(U_{j}^{0}\right)\right| .
$$

One finds that the proof of the analogous bound in [55] can be modified to accommodate the present situation. The key ingredients are the assumption that $\gamma_{1} A\left(u_{0}\right)_{x} \in B V(\mathbb{R})$, along with the pointwise discretization of $u_{0}$. For the proof of (4.60), see Lemma 3.4 of [55].

In what follows, it will be convenient to have available the notation $\mathcal{O}(1)$ to denote a quantity that is bounded uniformly in $n$ and $\Delta$.

Lemma 4.15. The following bound holds independently of $\Delta$ and the time level $n$ :

$$
\sum_{j \in \mathbb{Z}} s_{j-1 / 2}\left|\Delta_{-} A_{j}^{n}\right| \leq C
$$

Proof. Let $\rho_{j-1 / 2}^{n}:=h_{j-1 / 2}^{n}-s_{j-1 / 2} \Delta_{-} A_{j}^{n} / \Delta x$. By substituting $U_{j}^{n+1}-U_{j}^{n}=-\lambda \Delta_{+} \rho_{j-1 / 2}^{n}$ into (4.59) we find that

$$
\sum_{j \in \mathbb{Z}}\left|\Delta_{+} \rho_{j-1 / 2}^{n}\right|=\mathcal{O}(1) .
$$

At the same time, if $j$ is so large that $x_{j}>x_{\mathrm{R}}+2 \Delta x$, then $\rho_{j-1 / 2}^{n}=q_{\mathrm{R}} U_{j}^{n}$. From Lemma 4.12, we get that $\left|\rho_{j-1 / 2}^{n}\right| \leq q_{\mathrm{R}}$. This bound, together with the bound (4.62), implies a uniform bound of the form $\left|\rho_{j-1 / 2}^{n}\right|=\mathcal{O}(1)$. Since the convective numerical flux $h(\gamma, v, u)$ is continuous, the quantity $h_{j-1 / 2}^{n}$ is also uniformly bounded, and so we have the bound $s_{j-1 / 2}\left|\Delta_{-} A_{j}^{n}\right| / \Delta x=\mathcal{O}(1)$. The proof is completed by multiplying both sides of this relationship by $\Delta x$, summing over $j$, and recalling that $s_{j-1 / 2}$ vanishes for $x_{j}$ outside of the interval $\left[x_{\mathrm{L}}-\Delta x, x_{\mathrm{R}}\right]$.

Let $z^{\Delta}(x, t):=\Psi\left(\gamma(x), u^{\Delta}(x, t)\right)$. Defining (see (4.13) for the definition of $\mathcal{F}$ )

$$
\mathcal{F}^{\Delta}(x, t):=\mathcal{F}\left(\gamma(x), u^{\Delta}(x, t)\right), \quad A^{\Delta}(x, t):=A\left(u^{\Delta}(x, t)\right),
$$

we can separate $z^{\Delta}$ into its hyperbolic and parabolic contributions:

$$
z^{\Delta}(x, t)=\mathcal{F}^{\Delta}(x, t)+\gamma_{1}(x) A^{\Delta}(x, t) .
$$

To prove that the difference scheme converges, we follow [25] and first prove compactness for the transformed quantity $z^{\Delta}$. We establish spatial variation bounds separately for each of the intervals $\left(-\infty, x_{\mathrm{L}}\right),\left(x_{\mathrm{L}}, 0\right),\left(0, x_{\mathrm{R}}\right),\left(x_{\mathrm{R}}, \infty\right)$. The jumps in $z^{\Delta}$ where these intervals meet are bounded, and so we can ignore them. Indeed consider the jump in $z^{\Delta}\left(x, t^{n}\right)$ that occurs at $x=m \in\left\{x_{\mathrm{L}}, 0, x_{\mathrm{R}}\right\}$, which is given by

$$
z^{\Delta}\left(m+, t^{n}\right)-z^{\Delta}\left(m-, t^{n}\right)=\Psi\left(\gamma+, u^{\Delta}\left(m+, t^{n}\right)\right)-\Psi\left(\gamma-, u^{\Delta}\left(m-, t^{n}\right)\right) .
$$

Since $u^{\Delta}$ is bounded uniformly (by Lemma 4.12), $\gamma$ is bounded by assumption, and the transformation $\Psi$ is Lipschitz continuous with respect to all variables, it is clear that the magnitude of this jump is uniformly bounded also.

In the intervals $\left(-\infty, x_{\mathrm{L}}\right)$ and $\left(x_{\mathrm{R}}, \infty\right)$, the parabolic term is not present, and (4.63) simplifies to $z^{\Delta}(x, t)=\gamma_{2} u^{\Delta}(x, t)$. This makes it clear that the proof of the variation bound for these intervals is the same as the proof of Lemma 3.5 of [25]. We record this fact in the following lemma. 
Lemma 4.16. We have the following bounds, which are independent of $\Delta$ and $n$ :

$$
\operatorname{TV}\left(\left.z^{\Delta}\left(\cdot, t^{n}\right)\right|_{\left\{x \mid x<x_{\mathrm{L}}\right\}}\right) \leq C, \quad \operatorname{TV}\left(\left.z^{\Delta}\left(\cdot, t^{n}\right)\right|_{\left\{x \mid x>x_{\mathrm{R}}\right\}}\right) \leq C .
$$

We now address the variation bound for the remaining intervals, $\left(x_{\mathrm{L}}, 0\right)$ and $\left(0, x_{\mathrm{R}}\right)$. As in [25], we will focus on $\left(0, x_{\mathrm{R}}\right)$, omitting the proof for the other interval, since it is similar. Let $\gamma_{\mathrm{R}}:=\left(q_{\mathrm{R}}, 1\right)$, i.e., let $\gamma_{\mathrm{R}}$ denote the value that $\boldsymbol{\gamma}$ takes on $\left(0, x_{\mathrm{R}}\right)$. Recalling the definition (4.13), we see that $\gamma_{1}=1$ for $x \in\left(0, x_{\mathrm{R}}\right)$, and so $\Psi$ simplifies to

$$
\Psi(\gamma, u)=\mathcal{F}\left(\gamma_{\mathrm{R}}, u\right)+A(u), \quad \mathcal{F}\left(\gamma_{\mathrm{R}}, u\right)=\int_{0}^{u} \mathcal{S}(w)\left|f_{u}\left(\gamma_{\mathrm{R}}, w\right)\right| d w .
$$

Let $J^{-}$be the largest index $j$ such that $x_{j}-\Delta x / 2 \leq 0$, and let $J^{+}$be the smallest index $j$ such that $x_{j}+\Delta x / 2 \geq x_{\mathrm{R}}$. Thus $0 \in I_{J^{-}}, x_{\mathrm{R}} \in I_{J^{+}}$, and $\left[0, x_{\mathrm{R}}\right] \subseteq\left[x_{J^{-}}-\Delta x / 2, x_{J^{+}}+\Delta x / 2\right]$.

The following lemma records a discrete entropy inequality. It can be proved via a slight modification (to account for $s_{j-1 / 2}$ ) of the proof of Lemma 4.1 of [56].

Lemma 4.17. For any $c \in \mathbb{R}$, the following cell entropy inequality is satisfied by approximate solutions $U_{j}^{n}$ generated by the scheme (4.44):

$$
\begin{aligned}
\left|U_{j}^{n+1}-c\right| \leq\left|U_{j}^{n}-c\right| & -\lambda \Delta_{-} H_{j+1 / 2}^{n}+\mu \Delta_{+}\left(s_{j-1 / 2} \Delta_{-}\left|A\left(U_{j}^{n}\right)-A(c)\right|\right) \\
& -\lambda \operatorname{sgn}\left(U_{j}^{n+1}-c\right) \Delta_{+} f\left(\gamma_{j-1 / 2}, c\right)
\end{aligned}
$$

where the numerical entropy flux $H_{j+1 / 2}$ is defined by

$$
H_{j+1 / 2}^{n}:=h\left(\gamma_{j+1 / 2}, U_{j+1}^{n} \vee c, U_{j}^{n} \vee c\right)-h\left(\gamma_{j+1 / 2}, U_{j+1}^{n} \wedge c, U_{j}^{n} \wedge c\right) .
$$

Formally, the cell entropy inequality (4.66) can be motivated by assuming that the function $u$ in the integrand of (4.5) is piecewise constant on the rectangle $R_{j}^{n}:=\left(x_{j-1 / 2}, x_{j+1 / 2}\right) \times\left(t_{n}, t_{n+1}\right)$, and by choosing a sequence of test functions $\phi$ with support on $R_{j}^{n}$ that approximate the characteristic function $\chi_{j}^{n}$ of $R_{j}^{n}$. Moreover, the exact entropy flux defined in (4.12) is replaced by the numerical entropy flux (4.67). In this sense, the discrete entropy inequality (4.66) is consistent to the entropy inequality (4.5) for the exact solution, but observe that the term in the second line of (4.66), which mirrors the sum over $m \in \mathcal{J}$ in (4.5), is evaluated at time level $t_{n+1}$.

Let $\chi_{l}(w ; c):=H(c-w)$, where $H(\cdot)$ is the Heaviside function, and $\chi_{r}(w ; c):=1-\chi_{l}(w ; c)$. The following lemma is easily established using the calculations used in Lemma 3.9 of [25], adapted to the cell entropy inequality (4.66) appearing in Lemma 4.17.

Lemma 4.18. Fix $c \in \mathbb{R}$ and $\gamma \in \mathcal{G}$. The following inequalities are valid for $J^{-} \leq j \leq J^{+}$:

$$
\begin{aligned}
& -\int_{U_{j}^{n}}^{U_{j+1}^{n}} \chi_{l}(w ; c) f_{u}^{-}(\gamma, w) d w-\int_{U_{j-1}^{n}}^{U_{j}^{n}} \chi_{l}(w ; c) f_{u}^{+}(\gamma, w) d w \\
& \leq \frac{-1}{\lambda}\left(U_{j}^{n}-U_{j}^{n+1}\right)_{-}-\frac{1}{\Delta x} \Delta_{+}\left(s_{j-1 / 2} \Delta_{-}\left(A\left(U_{j}^{n+1}\right)-A(c)\right)_{-}\right)+\alpha_{j}^{n}, \\
& \int_{U_{j}^{n}}^{U_{j+1}^{n}} \chi_{r}(w ; c) f_{u}^{-}(\gamma, w) d w+\int_{U_{j-1}^{n}}^{U_{j}^{n}} \chi_{r}(w ; c) f_{u}^{+}(\gamma, w) d w \\
& \leq \frac{1}{\lambda}\left(U_{j}^{n}-U_{j}^{n+1}\right)_{+}-\frac{1}{\Delta x} \Delta_{+}\left(s_{j-1 / 2} \Delta_{-}\left(A\left(U_{j}^{n+1}\right)-A(c)\right)_{+}\right)+\beta_{j}^{n} .
\end{aligned}
$$

The quantities $\alpha_{j}^{n}$ and $\beta_{j}^{n}$ are bounded independently of $n$ and $\Delta$. In fact, $\alpha_{j}^{n}=\beta_{j}^{n}=0$ for $J^{-}+2 \leq j \leq J^{+}-2$.

With the help of these entropy inequalities, we can prove the following lemma.

Lemma 4.19. The following spatial variation bounds are satisfied, independent of $\Delta$ and $n$ :

$$
\operatorname{TV}\left(\left.\mathcal{F}^{\Delta}\left(\cdot, t^{n}\right)\right|_{\left\{x \mid x_{\mathrm{L}}<x<0\right\}}\right) \leq C, \quad \operatorname{TV}\left(\left.\mathcal{F}^{\Delta}\left(\cdot, t^{n}\right)\right|_{\left\{x \mid 0<x<x_{\mathrm{R}}\right\}}\right) \leq C .
$$


Proof. We only prove the second assertion; the proof of the first is similar. We follow closely the proof of Lemma 3.10 of [25]. First, observe that if the term

$$
\frac{1}{\Delta x} \Delta_{-}\left(\Delta_{+} s_{j-1 / 2}\left(A\left(U_{j}^{n}\right)-A(c)\right)_{-}\right)
$$

was not present in (4.68), the proof of Lemma 3.10 of [25] would apply verbatim. Next, recall from the proof of Lemma 4.3 that if $c \leq u_{\mathrm{c}}$, then $\left(A\left(U_{j}^{n}\right)-A(c)\right)_{-}=0$. Thus, when $c \leq u_{\mathrm{c}}$, the parabolic term in (4.68) disappears, giving us

$$
-\int_{U_{j}^{n}}^{U_{j+1}^{n}} \chi_{l}(w ; c) f_{u}^{-}(\boldsymbol{\gamma}, w) d w-\int_{U_{j-1}^{n}}^{U_{j}^{n}} \chi_{l}(w ; c) f_{u}^{+}(\boldsymbol{\gamma}, w) d w \leq \frac{-1}{\lambda}\left(U_{j}^{n}-U_{j}^{n+1}\right)_{-}+\alpha_{j}^{n} .
$$

When $x \in\left(0, x_{\mathrm{R}}\right),\left(\gamma_{1}(x), \gamma_{2}(x)\right)=\left(q_{\mathrm{R}}, 1\right) \equiv \gamma_{\mathrm{R}}$, and by the assumptions on $b$ and $q_{\mathrm{R}}, u \mapsto$ $f\left(\gamma_{R}, u\right)$ has at most two extrema for $u \in(0,1)$. For the sake of argument, we assume that there are exactly two extrema. It will become clear that a simplified version of the following proof will suffice if there are fewer than two. So assume that there is one maximum located at $u_{1}^{*} \in(0,1)$, and one minimum located at $u_{2}^{*} \in(0,1)$, with $u_{1}^{*}<u_{2}^{*}$. The flux $u \mapsto f\left(\gamma_{\mathrm{R}}, u\right)$ is strictly monotone away from these critical points. Let $u_{0}^{*}:=0, u_{3}^{*}:=1$ and for $\nu=0,1,2$, let $\chi^{\nu}(u)$ be the characteristic function of the interval $\left[\min \left\{u_{\nu}^{*}, u_{\mathrm{c}}\right\}, \min \left\{u_{\nu+1}^{*}, u_{\mathrm{c}}\right\}\right)$. Each of the intervals $\left[\min \left\{u_{\nu}^{*}, u_{\mathrm{c}}\right\}, \min \left\{u_{\nu+1}^{*}, u_{\mathrm{c}}\right\}\right.$ ) is either empty (if the left endpoint happens to equal $u_{\mathrm{c}}$ ), or $f\left(\gamma_{\mathrm{R}}, u\right)$ is strictly monotone in its interior. Define

$$
\phi^{\nu}\left(\boldsymbol{\gamma}_{\mathrm{R}}, u\right):=\int_{0}^{u} \chi^{\nu}(w)\left|f_{u}\left(\boldsymbol{\gamma}_{\mathrm{R}}, w\right)\right| d w, \quad \nu=0,1,2 .
$$

Clearly, $\mathcal{S}(u)=\chi^{0}(u)+\chi^{1}(u)+\chi^{2}(u)$, so that $\mathcal{F}\left(\gamma_{R}, \cdot\right)$ has the decomposition

$$
\mathcal{F}\left(\gamma_{\mathrm{R}}, u\right)=\phi^{0}\left(\gamma_{\mathrm{R}}, u\right)+\phi^{1}\left(\boldsymbol{\gamma}_{\mathrm{R}}, u\right)+\phi^{2}\left(\gamma_{\mathrm{R}}, u\right) .
$$

We now use the entropy inequality (4.70) three times, just as in the proof of Lemma 3.10 of [25], except that now instead of $c=u_{\nu}, \nu=1,2,3$, we take $c=\min \left\{u_{\nu}^{*}, u_{\mathrm{c}}\right\}, \nu=1,2,3$. In order to keep the analysis somewhat self-contained, let us review the calculation appearing in [25] when $c=u_{1}^{*}$. We start by setting $c=u_{1}^{*}$ in inequality (4.70), and observe that $u \mapsto f\left(\gamma_{\mathrm{R}}, u\right)$ is strictly increasing on $\left(0, u_{1}^{*}\right)$. Then (4.70) simplifies to

$$
-\int_{U_{j-1}^{n}}^{U_{j}^{n}} \chi_{l}\left(w ; u_{1}^{*}\right) f_{u}^{+}\left(\gamma_{\mathrm{R}}, w\right) d w \leq \frac{-1}{\lambda}\left(U_{j}^{n}-U_{j}^{n+1}\right)_{-}+\alpha_{j}^{n} .
$$

Since $f_{u}^{+}\left(\gamma_{\mathrm{R}}, u\right)=\left|f_{u}\left(\gamma_{\mathrm{R}}, u\right)\right|$ for $u \in\left(0, u_{1}^{*}\right)$, we find that

$$
\int_{U_{j-1}^{n}}^{U_{j}^{n}} \chi_{l}\left(w ; u_{1}^{*}\right) f_{u}^{+}\left(\gamma_{\mathrm{R}}, w\right) d w=\int_{U_{j-1}^{n}}^{U_{j}^{n}} \chi^{0}(w)\left|f_{u}\left(\boldsymbol{\gamma}_{\mathrm{R}}, w\right)\right| d w=\phi^{0}\left(\gamma_{\mathrm{R}}, U_{j}^{n}\right)-\phi^{0}\left(\gamma_{\mathrm{R}}, U_{j-1}^{n}\right) .
$$

Combining this last relationship with (4.72), we have the inequality

$$
\phi^{0}\left(\gamma_{\mathrm{R}}, U_{j-1}^{n}\right)-\phi^{0}\left(\gamma_{\mathrm{R}}, U_{j}^{n}\right) \leq \frac{1}{\lambda}\left|U_{j}^{n+1}-U_{j}^{n}\right|+\alpha_{j}^{n},
$$

and, since the right side of this inequality is nonnegative, we also have

$$
-\left(\phi^{0}\left(\gamma_{\mathrm{R}}, U_{j}^{n}\right)-\phi^{0}\left(\gamma_{\mathrm{R}}, U_{j-1}^{n}\right)\right)_{-} \leq \frac{1}{\lambda}\left|U_{j}^{n+1}-U_{j}^{n}\right|+\alpha_{j}^{n} .
$$

Next, we sum (4.73) over $j$ and invoke Lemmas 4.18 and 4.14 to obtain

$$
\begin{aligned}
& -\sum_{j=J^{-}}^{J^{+}}\left(\phi^{0}\left(\gamma_{\mathrm{R}}, U_{j}^{n}\right)-\phi^{0}\left(\gamma_{\mathrm{R}}, U_{j-1}^{n}\right)\right)_{-} \leq \sum_{j=J^{-}}^{J^{+}}\left(\frac{1}{\lambda}\left|U_{j}^{n+1}-U_{j}^{n}\right|+\left|\alpha_{j}^{n}\right|\right) \\
& \leq \sum_{j \in \mathbb{Z}} \frac{1}{\lambda}\left|U_{j}^{n+1}-U_{j}^{n}\right|+\left|\alpha_{J^{+}}^{n}\right|+\left|\alpha_{J^{+-1}}^{n}\right|+\left|\alpha_{J^{-}}^{n}\right|+\left|\alpha_{J^{-}+1}^{n}\right|=\mathcal{O}(1) .
\end{aligned}
$$


Finally, we observe that since $\phi^{0}$ is bounded uniformly in $\Delta$ and $n$, it follows from this bound on the negative variation that $\phi^{0}$ also has uniformly bounded total variation. Similar calculations (see [25]) result in uniform bounds on the total variation of $\phi^{1}$ and $\phi^{2}$, i.e., we have

$$
\sum_{j=J^{-}}^{J^{+}}\left|\phi^{\nu}\left(\gamma_{\mathrm{R}}, U_{j}^{n}\right)-\phi^{\nu}\left(\gamma_{\mathrm{R}}, U_{j-1}^{n}\right)\right|=\mathcal{O}(1), \quad \nu=0,1,2 .
$$

In view of (4.71), the proof is completed by combining these three bounds.

With this spatial variation bound established, we can prove the following lemma. We omit the proof, which is not essentially different from the proof of Lemma 3.8 of [55].

Lemma 4.20. There exists a subsequence of $\left\{\mathcal{F}^{\Delta}\right\}$, also denoted by $\left\{\mathcal{F}^{\Delta}\right\}$, and a function $\overline{\mathcal{F}} \in$ $L^{1}\left(\Pi_{T}\right) \cap L^{\infty}\left(\Pi_{T}\right)$ such that $\mathcal{F}^{\Delta} \rightarrow \overline{\mathcal{F}}$ in $L_{\mathrm{loc}}^{1}\left(\Pi_{T}\right)$ and a.e. in $\Pi_{T}$. Furthermore, $\overline{\mathcal{F}}(\cdot, t) \in L^{1}(\mathbb{R})$ for all $t \in[0, T]$.

The following lemma establishes convergence (along a subsequence) of the discrete diffusion term $A^{\Delta}$. The proof is similar to the proofs of Lemmas 3.9, 3.10, 3.11, and 3.12 of [55].

Lemma 4.21. The following bounds are satisfied, independent of $n$ and $\Delta$ :

$$
\begin{aligned}
& \left\{\Delta t \Delta x \sum_{n \geq 0} \sum_{j \in \mathbb{Z}} s_{j+1 / 2}\left(\Delta_{+} A\left(U_{j}^{n}\right)\right)^{2}\right\}^{1 / 2} \leq C \Delta x, \\
& \left\|A\left(u^{\Delta}(\cdot+y, \cdot)\right)-A\left(u^{\Delta}(\cdot, \cdot)\right)\right\|_{L^{2}\left(\Omega_{y}\right)} \leq C \sqrt{|y|(|y|+\Delta x)}, \quad \forall y \in\left(x_{\mathrm{L}}, x_{\mathrm{R}}\right), \\
& \left\|A\left(u^{\Delta}(\cdot, \cdot+\tau)\right)-A\left(u^{\Delta}(\cdot, \cdot)\right)\right\|_{L^{2}\left(\Omega_{\tau}\right)} \leq C \sqrt{\tau+\Delta t}, \quad \forall \tau \in(0, T),
\end{aligned}
$$

where $\Omega_{y}$ consists of all $(x, t) \in \Pi_{T}$ such that $x$ and $x+y$ belong to $\left(x_{\mathrm{L}}, x_{\mathrm{R}}\right) \times(0, T)$ and $\Omega_{\tau}:=$ $\left.\left(x_{\mathrm{L}}, x_{\mathrm{R}}\right) \times(0, T-\tau)\right)$. Finally, we have that there exists a subsequence of $\left\{A^{\Delta}\right\}$, also denoted by $\left\{A^{\Delta}\right\}$, and a function $\bar{A} \in L^{2}\left(0, T ; H^{1}\left(x_{\mathrm{L}}, x_{\mathrm{R}}\right)\right)$ such that $A^{\Delta} \rightarrow \bar{A}$ in $L^{2}\left(\left(x_{\mathrm{L}}, x_{\mathrm{R}}\right) \times(0, T)\right.$ and boundedly a.e. in $\left(x_{\mathrm{L}}, x_{\mathrm{R}}\right) \times(0, T)$. Furthermore, $\bar{A}=A(u)$ a.e. in $\left(x_{\mathrm{L}}, x_{\mathrm{R}}\right) \times(0, T)$, where $u$ denotes the $L^{\infty}$ weak-* limit of $u^{\Delta}$.

It is possible to establish more regularity of the diffusion function than displayed in Lemma 4.21. This additional regularity, which is stated in the lemma below, will be used later in the proof of Theorem 4.2 .

Lemma 4.22. There exists a constant $C$, independent of $\Delta$, such that

$$
\left|A\left(U_{j}^{n}\right)-A\left(U_{i}^{n}\right)\right| \leq C|j-i| \Delta x, \quad\left|A\left(U_{j}^{n}\right)-A\left(U_{j}^{m}\right)\right| \leq C \sqrt{|n-m| \Delta t}
$$

for all $i, j, n, m$ such that $\left(x_{i}, t_{n}\right),\left(x_{j}, t_{n}\right)$, and $\left(x_{j}, t_{m}\right)$ belong to $\left(x_{\mathrm{L}}, x_{\mathrm{R}}\right) \times(0, T)$.

Define $\tilde{A}^{n}(x)$ as

$$
\tilde{A}^{n}(x)=\frac{1}{\Delta x}\left(\left(x-x_{j-1}\right) A\left(U_{j}^{n}\right)+\left(x_{j}-x\right) A\left(U_{j-1}^{n}\right)\right), \quad x \in\left[x_{j-1}, x_{j}\right] .
$$

Then define

$$
\tilde{A}^{\Delta}(x, t)=\frac{1}{\Delta t}\left(\left(t-t_{n}\right) \tilde{A}^{n+1}(x)+\left(t_{n+1}-t\right) \tilde{A}^{n}(x)\right), \quad t \in\left[t_{n}, t_{n+1}\right] .
$$

Then there exists a subsequence of $\tilde{A}^{\Delta}$, also denoted by $\tilde{A}^{\Delta}$, and a function

$$
\tilde{A} \in C^{1,1 / 2}\left(\left(x_{\mathrm{L}}, x_{\mathrm{R}}\right) \times(0, T)\right)
$$

such that $\tilde{A}^{\Delta} \rightarrow \bar{A}$ in $L^{\infty}\left(\left(x_{\mathrm{L}}, x_{\mathrm{R}}\right) \times(0, T)\right)$. Moreover, there holds $\left(\tilde{A}^{\Delta}\right)_{x} \stackrel{\star}{\rightarrow} \tilde{A}_{x}$ in $L^{\infty}\left(\left(x_{\mathrm{L}}, x_{\mathrm{R}}\right) \times\right.$ $(0, T))$.

This lemma can be proved by a straightforward adaptation of the proofs of Lemmas 4.1 and 4.2 and Theorem 4.1 in [55].

We can now prove our main convergence theorem. 
Theorem 4.2. Assume that the hypotheses concerning the data stated in Section 4.1 are satisfied. Then there exists a $B V_{t}$ weak solution of the initial value problem (4.1) that satisfies the entropy condition (D.5). Let $u^{\Delta}$ be defined by (3.3) and the scheme (4.44), with the parameters $\Delta x$ and $\Delta t$ chosen so that the CFL condition (4.46) holds. Then, along a subsequence, $u^{\Delta} \rightarrow u$ in $L_{\mathrm{loc}}^{1}\left(\Pi_{T}\right)$ and a.e. in $\Pi_{T}$, where $u$ is a $B V_{t}$ weak solution.

Proof. The proof of convergence (along a subsequence) to a function $u: \Pi_{T} \rightarrow \mathbb{R}$ is essentially the same as the proof of Theorem 3.1 of [55]. The main idea is to observe that $z^{\Delta}=\Psi\left(\gamma^{\Delta}, u^{\Delta}\right)=$ $\mathcal{F}^{\Delta}+\gamma_{1} A^{\Delta}$. Convergence (along a subsequence) of $\left\{z^{\Delta}\right\}$ then follows from compactness for the sequences $\mathcal{F}^{\Delta}$ and $\gamma_{1} A^{\Delta}$ (Lemmas 4.20 and 4.21). Letting $z(x, t)$ denote $\lim _{\Delta \rightarrow 0} z^{\Delta}(x, t)$, one then recovers the conserved quantity $u$ via $u(x, t)=\Psi^{-1}(\gamma(x), z(x, t))$. The arguments in [55] also (with some slight modifications to account for $\gamma_{1}$ multiplying $A(u)_{x}$ ) show that $u \in$ $L^{1}\left(\Pi_{T}\right) \cap L^{\infty}\left(\Pi_{T}\right) \cap C\left(0, T ; L^{1}(\mathbb{R})\right)$ and $A(u) \in L^{2}\left(0, T ; H^{1}\left(x_{\mathrm{L}}, x_{\mathrm{R}}\right)\right)$. It follows readily from the discrete time continuity estimate (4.59) that $u \in B V_{t}\left(\Pi_{T}\right)$ (see the proof of Theorem 3.1 of [25]), and that the initial data is assumed in the strong $L^{1}$ sense, i.e, (4.4) is satisfied.

To show that the limit $u$ is a $B V_{t}$ weak solution, it remains to verify that the weak formulation (4.3) is satisfied, for which a a Lax-Wendroff type calculation is required. The proof of Theorem 3.1 of [55] applies in the present situation, with the exception that the spatially varying coefficient $s_{j-1 / 2}$ multiplying the parabolic term causes some new complications. We can lay this matter to rest if we can show that for $\phi \in \mathcal{D}\left(\Pi_{T}\right)$, and with $\phi_{j}^{n}:=\phi\left(x_{j}, t_{n}\right)$,

$$
\Delta x \Delta t \sum_{n \geq 0} \sum_{j \in \mathbb{Z}} \frac{1}{\Delta x^{2}} \Delta_{+}\left(s_{j-1 / 2} \Delta_{-} A_{j}^{n}\right) \phi_{j}^{n} \rightarrow-\iint_{\Pi_{T}} \gamma_{1}(x) A(u)_{x} \phi_{x} d x d t .
$$

Summing by parts, we get the following expression for the left-hand side of (4.76):

$$
-\Delta x \Delta t \sum_{n \geq 0} \sum_{j \in \mathbb{Z}} \frac{1}{\Delta x}\left(s_{j-1 / 2} \Delta_{-} A_{j}^{n}\right)\left(\Delta_{-} \phi_{j}^{n} / \Delta x\right) .
$$

Let $\tilde{A}^{\Delta}$ be the interpolant defined in Lemma 4.22. Observe that $\left(\tilde{A}^{\Delta}\right)_{x}=\Delta_{+} A\left(U_{j}^{n}\right)$ on the parallelogram $P_{j}^{n}$ with vertices $\left(x_{j}, t^{n-1}\right),\left(x_{j}, t^{n}\right),\left(x_{j+1}, t^{n}\right)$, and $\left(x_{j+1}, t^{n+1}\right)$.

We now have

$$
\begin{aligned}
-\Delta & x \Delta \sum_{n \geq 0} \sum_{j \in \mathbb{Z}} \frac{1}{\Delta x}\left(s_{j-1 / 2} \Delta_{-} A_{j}^{n}\right)\left(\Delta_{-} \phi_{j}^{n} / \Delta x\right) \\
= & \sum_{n \geq 0} \sum_{j \in \mathbb{Z}} \iint_{P_{j}^{n+1}} \gamma_{1}(x)\left(\tilde{A}^{\Delta}\right)_{x} \phi_{x} d x d t+\mathcal{O}(\Delta x+\Delta t) \\
= & \iint_{\Pi_{T}} \gamma_{1}(x)\left(\tilde{A}^{\Delta}\right)_{x} \phi_{x} d x d t+\mathcal{O}(\Delta x+\Delta t) .
\end{aligned}
$$

According to Lemma 4.22 we can assume that $\tilde{A}^{\Delta} \rightarrow \bar{A}$ in $L^{\infty}\left(\left(x_{\mathrm{L}}, x_{\mathrm{R}}\right) \times(0, T)\right)$. Since $u^{\Delta} \rightarrow u$ a.e. in $\Pi_{T}$, we can repeat the proof of Theorem 4.1 in [55] to show that $\tilde{A}=A(u)$ a.e. in $\left(x_{\mathrm{L}}, x_{\mathrm{R}}\right) \times(0, T)$. Recall that the parameter $\gamma_{1}(x)$ takes the value 1 for $x \in\left(x_{\mathrm{L}}, x_{\mathrm{R}}\right)$ and is zero elsewhere. Using this and the convergence $\left(\tilde{A}^{\Delta}\right)_{x} \stackrel{\star}{\rightarrow} A(u)_{x}$ in $L^{\infty}\left(\left(x_{\mathrm{L}}, x_{\mathrm{R}}\right) \times(0, T)\right)$ when sending $\Delta \rightarrow 0$ in (4.78), we get (4.76).

The proof that $u$ satisfies the entropy inequality (4.5) requires another Lax-Wendroff type calculation, this time based on the cell entropy inequality (4.66). The proof of Theorem 5.1 of [55], or Lemma 4.1 of [56], suffices for the situation at hand, again with the exception of the parabolic terms, due to the presence of $s_{j-1 / 2}$. It is possible to resolve this matter by an argument (which we omit) similar to the one above.

Remark 4.3. In this proof, we have verified all but condition (D.4) of Definition 4.1. Thus, if we one were able to prove that (D.4) is satisfied, then the limit $u$ of Theorem 4.2 would be the $B V_{t}$ entropy solution whose uniqueness is guaranteed by Theorem 4.1. Although our numerical results suggest that this condition is satisfied by the limit of the sequence of approximate solutions, a rigorous proof of this property is still left as an open problem. 


\section{SteAdy-STATE SOlUtions}

The construction of steady states is based on the stationary version of (2.16) or (2.21). We do not present here a thorough analysis of all steady states but identify some stationary solutions in order to motivate the choices of the control parameters for the transient simulations. Our construction of steady states will follow a procedure similar to that of the simpler continuous thickening models treated in $[16,17]$. Specifically, we fix the material model, the vessel geometry, and assume that the clarifier-thickener is to be operated at given values of $Q_{\mathrm{L}}, Q_{\mathrm{F}}$ and $u_{\mathrm{F}}$, and is supposed to produce a thickened sediment of a discharge concentration $u_{\mathrm{D}}>u_{\mathrm{c}}$. Although the construction given below can be extended in a straightforward manner to vessels with varying crosssectional area, there are some subtle details that require that we restrict the rigorous discussion to vessels with constant cross-sectional interior area, so that we limit the discussion to Model 1.

Our notation is consistent to Section 4, i.e., we refer to the space variable by $x$ (instead of $w$ ), to the solution by $u$ (instead of $v$ ) and to the integrated diffusion coefficient by $A$ (instead of $\mathcal{A}$ ).

Definition 5.1. A piecewise twice differentiable function $u: \mathbb{R} \rightarrow\left[0, u_{\max }\right]$ is a steady-state entropy weak solution of Model 1 if the following conditions are satisfied: (a) the function $\gamma_{1}(x) A(u)^{\prime}$ is bounded, where' $=d / d x$; (b) the function $u$ is a weak solution to the following ordinary differential equation that arises from (2.21), and where $g(x, u)$ is given by (2.23):

$$
g(x, u)^{\prime}=\left(\gamma_{1}(x) A(u)^{\prime}\right)^{\prime},
$$

i.e., for every test function $\phi \in C_{0}^{2}(\mathbb{R})$ with compact support we have

$$
\int_{\mathbb{R}}\left(f(\gamma(x), u(x))-\gamma_{1}(x) A(u(x))^{\prime}\right) \phi^{\prime}(x) d x=0,
$$

and (c) the following entropy inequality holds for all test functions $\phi \in C_{0}^{2}(\mathbb{R}), \phi \geq 0$ and $k \in \mathbb{R}$ :

$$
\begin{aligned}
& \int_{\mathbb{R}}\left(\operatorname{sgn}(u(\xi)-k)(f(\gamma(\xi), u(\xi))-f(\gamma(\xi), k))-\gamma_{1}(\xi) A(u)^{\prime}\right) \phi^{\prime}(\xi) d \xi \\
& +\sum_{m \in \mathcal{J}}\left|f\left(\gamma\left(m^{+}\right), k\right)-f\left(\gamma\left(m^{-}\right), k\right)\right| \phi(m) \geq 0 .
\end{aligned}
$$

It is standard to conclude from (5.2) that the following jump condition has to be satisfied across any discontinuity of the steady-state solution, where $u\left(x^{+}\right)$and $u\left(x^{-}\right)$refer to limits of $u(\xi)$ taken for $\xi \rightarrow x$ with $\xi>x$ and $\xi<x$, respectively:

$$
f\left(\gamma\left(x^{-}\right), u\left(x^{-}\right)\right)-\left.\gamma_{1}\left(x^{-}\right) A^{\prime}(u)\right|_{x=x^{-}}=f\left(\gamma\left(x^{+}\right), u\left(x^{+}\right)\right)-\left.\gamma_{1}\left(x^{+}\right) A^{\prime}(u)\right|_{x=x^{+}} .
$$

It is easy to see that this condition implies that steady-state solutions are constant for $x<x_{\mathrm{L}}$ and $x>x_{\mathrm{R}}$.

Lemma 5.1. Inequality (5.3) implies the following entropy jump condition:

$$
\begin{aligned}
\forall k \in \mathbb{R}: \quad & \operatorname{sgn}\left(u\left(x^{+}\right)-k\right)\left[f\left(\gamma\left(x^{+}\right), u\left(x^{+}\right)\right)-f\left(\gamma\left(x^{+}\right), k\right)-\left.\gamma_{1}\left(x^{+}\right) A^{\prime}(u)\right|_{x=x^{+}}\right] \\
- & \operatorname{sgn}\left(u\left(x^{-}\right)-k\right)\left[f\left(\gamma\left(x^{-}\right), u\left(x^{-}\right)\right)-f\left(\gamma\left(x^{-}\right), k\right)-\left.\gamma_{1}\left(x^{-}\right) A^{\prime}(u)\right|_{x=x^{-}}\right] \\
& \leq\left|f\left(\gamma\left(x^{+}\right), k\right)-f\left(\gamma\left(x^{-}\right), k\right)\right| .
\end{aligned}
$$

(Note that the right-hand part of (5.5) is zero for $x \notin \mathcal{J}$.)

Proof. The proof is a simpler variant of the proof of Lemma 2.6 of [56]. To outline it, let us fix $m \in \mathcal{J}=\left\{x_{\mathrm{L}}, 0, x_{\mathrm{R}}\right\}$, and define the function

$$
\theta_{\varepsilon}(x):= \begin{cases}(\varepsilon+x) / \varepsilon & \text { if } x \in[-\varepsilon, 0] \\ (\varepsilon-x) / \varepsilon & \text { if } x \in[0, \varepsilon], \\ 0 & \text { otherwise }\end{cases}
$$


with a parameter $\varepsilon>0$. A density argument will reveal that we may choose the compactly supported Lipschitz continuous function $\theta_{\varepsilon}(x-m)$ as a test function in (5.3). This yields

$$
\begin{gathered}
\frac{1}{\varepsilon} \int_{m-\varepsilon}^{m}\left(F(\gamma(\xi), u(\xi), k)-\gamma_{1}(x)|A(u)-A(k)|^{\prime}\right) d x \\
-\frac{1}{\varepsilon} \int_{m}^{m+\varepsilon}\left(F(\gamma(\xi), u(\xi), k)-\gamma_{1}(x)|A(u)-A(k)|^{\prime}\right) d x \\
+\left|f\left(\gamma\left(m^{+}\right), k\right)-f\left(\gamma\left(m^{-}\right), k\right)\right| \geq 0,
\end{gathered}
$$

where we recall the notation (4.12). Since the solution $u(x)$ is piecewise smooth, we may apply a time-independent version of Lemma 4.7 to conclude that for $\varepsilon \rightarrow 0$, we obtain from (5.6)

$$
\begin{aligned}
& F\left(\gamma\left(m^{+}\right), u\left(m^{+}\right), k\right)-\operatorname{Lim}_{\xi \downarrow m}\left(\gamma_{1}(\xi)|A(u)-A(k)|^{\prime}\right) \\
& -F\left(\gamma\left(m^{-}\right), u\left(m^{-}\right), k\right)+\operatorname{Lim}_{\xi \uparrow m}\left(\gamma_{1}(\xi)|A(u)-A(k)|^{\prime}\right) \leq\left|f\left(\gamma\left(m^{+}\right), k\right)-f\left(\gamma\left(m^{-}\right), k\right)\right| .
\end{aligned}
$$

Assume for the moment that $u\left(m^{+}\right) \neq k$ and $u\left(m^{-}\right) \neq k$. Then Lemma 4.7 implies

$$
\begin{aligned}
& \mathrm{L}_{\xi \downarrow m}\left(\gamma_{1}(\xi)|A(u)-A(k)|^{\prime}\right)=\left.\operatorname{sgn}\left(u\left(m^{+}\right)-k\right) \gamma_{1}\left(m^{+}\right) A^{\prime}(u)\right|_{x=m^{+}}, \\
& \mathrm{L}_{\xi \uparrow m}\left(\gamma_{1}(\xi)|A(u)-A(k)|^{\prime}\right)=\left.\operatorname{sgn}\left(u\left(m^{-}\right)-k\right) \gamma_{1}\left(m^{-}\right) A^{\prime}(u)\right|_{x=m^{-}},
\end{aligned}
$$

such that (5.7) already implies (5.5). To remove this restriction, assume that $k=u\left(m^{-}\right)$(the other case is similar). Then the left-hand side of (5.5) is just

$$
L:=\operatorname{sgn}\left(u\left(m^{+}\right)-u\left(m^{-}\right)\right)\left[f\left(\gamma\left(m^{+}\right), u\left(m^{+}\right)\right)-f\left(\gamma\left(m^{+}\right), u\left(m^{-}\right)\right)-\left.\gamma_{1}\left(m^{+}\right) A^{\prime}(u)\right|_{x=m^{+}}\right] .
$$

Using the jump condition (5.4), we obtain

$$
\begin{aligned}
L & =\operatorname{sgn}\left(u\left(m^{+}\right)-u\left(m^{-}\right)\right)\left[f\left(\gamma\left(m^{-}\right), u\left(m^{-}\right)\right)-f\left(\gamma\left(m^{+}\right), u\left(m^{-}\right)\right)-\left.\gamma_{1}\left(m^{-}\right) A^{\prime}(u)\right|_{x=m^{-}}\right] \\
& \leq\left|f\left(\gamma\left(m^{-}\right), u\left(m^{-}\right)\right)-f\left(\gamma\left(m^{+}\right), u\left(m^{-}\right)\right)\right|-\left.\operatorname{sgn}\left(u\left(m^{+}\right)-u\left(m^{-}\right)\right) \gamma_{1}\left(m^{-}\right) A^{\prime}(u)\right|_{x=m^{-}},
\end{aligned}
$$

and finally applying a steady-state variant of the right inequality of (4.27) in Lemma 4.8, we get

$$
L \leq\left|f\left(\gamma\left(m^{-}\right), u\left(m^{-}\right)\right)-f\left(\gamma\left(m^{+}\right), u\left(m^{-}\right)\right)\right|,
$$

which is the inequality (5.5). Finally, since we are dealing with time-independent solutions, inequality (5.6) and the remaining discussion remain valid if we replace $m \in \mathcal{J}$ by $x \in \mathbb{R}$.

The following lemma states a useful continuity result.

Lemma 5.2. Let $u(x)$ be a piecewise differentiable steady-state entropy weak solution of Model 1 . Then $A\left(u\left(x^{+}\right)\right)=A\left(u\left(x^{-}\right)\right)$for all $x \in \mathbb{R}$.

Proof. We consider first a point $x \in\left(x_{\mathrm{L}}, x_{\mathrm{R}}\right)$, at which $\gamma_{1}$ is continuous. Then the boundedness of $\gamma_{1}(x) A(u)^{\prime}$ implies that

$$
0=\lim _{\varepsilon \rightarrow 0} \int_{x-\varepsilon}^{x+\varepsilon} \gamma_{1}(\xi) A(u(\xi))^{\prime} d \xi=\lim _{\varepsilon \rightarrow 0} \int_{x-\varepsilon}^{x+\varepsilon} A(u(\xi))^{\prime} d \xi=A\left(u\left(x^{+}\right)\right)-A\left(u\left(x^{-}\right)\right) .
$$

Furthermore, consider that boundedness of $\gamma_{1}(x) A(u)^{\prime}$ implies that $A(u)^{\prime}$ is uniformly bounded on $\left[x_{\mathrm{L}}, x_{\mathrm{R}}\right]$. On the other hand, for $x<x_{\mathrm{L}}$ and $x>x_{\mathrm{R}}$, the jump condition (5.4) reduces to $q_{\mathrm{L}} u\left(x^{-}\right)=q_{\mathrm{L}}\left(x^{+}\right)$and $q_{\mathrm{R}} u\left(x^{+}\right)=q_{\mathrm{R}} u\left(x^{+}\right)$, respectively, which implies $u\left(x^{-}\right)=u\left(x^{+}\right)$and therefore no jumps are possible for $x<x_{\mathrm{L}}$ and $x>x_{\mathrm{R}}$. We conclude that piecewise smooth steady-state solutions are constant on $\left(-\infty, x_{\mathrm{L}}\right)$ and $\left(x_{\mathrm{R}}, \infty\right)$, and therefore we have $A(u)^{\prime}=0$ on $\left(-\infty, x_{\mathrm{L}}\right) \cup\left(x_{\mathrm{R}}, \infty\right)$. Thus $A(u)^{\prime}$ is uniformly bounded, and therefore has a continuous primitive $A(u)$, which implies that $A\left(u\left(x^{+}\right)\right)=A\left(u\left(x^{-}\right)\right)$for all $x \in \mathbb{R}$.

Remark 5.1. We point out that the continuity property established by Lemma 5.2 includes the steady-state analogue of condition (D.4) stated for the time-dependent Model 1. However, we see that for our class of steady-state solutions, the continuity of $A(u)$ across $x=x_{\mathrm{L}}$ and $x=x_{\mathrm{R}}$ is a result of a more general regularity property, and need not be postulated separately. 
Constructively, we proceed by selecting the discharge concentration $u_{\mathrm{D}}=\left.u(x)\right|_{x>x_{\mathrm{R}}}$, integrate the ODE arising from the steady-state version of Model 1 upwards, by obeying jump conditions wherever necessary. In doing so, we shall establish the limitations the entropy condition imposes on the choice of control parameters. Thus, the one-sided boundary condition is

$$
u\left(x_{\mathrm{R}}^{-}\right)=u_{\mathrm{D}}>u_{\mathrm{c}} .
$$

The discussion will be limited to those cases where the compression zone does not reach the overflow level. In addition, to further simplify the discussion, we assume that the functions $g_{\mathrm{L}}(u):=q_{\mathrm{L}} u+b(u)$ and $g_{\mathrm{R}}(u):=q_{\mathrm{R}} u+b(u)$ are monotone on the interval $\left[0, u_{\mathrm{c}}\right]$, i.e.,

$$
q_{\mathrm{L}}+b^{\prime}(u)>0, \quad q_{\mathrm{R}}+b^{\prime}(u)>0 \quad \text { for } u \in\left[0, u_{\mathrm{c}}\right] .
$$

Moreover, we limit ourselves to steady-state solutions for which the overflow or effluent concentration $u_{\mathrm{E}}:=\left.u(x)\right|_{x<x_{\mathrm{L}}}$ is zero, that is, we choose the parameters $u_{\mathrm{D}}$ and $u_{\mathrm{F}}$ such that

$$
Q_{\mathrm{F}} u_{\mathrm{F}}=\left(Q_{\mathrm{R}}-Q_{\mathrm{L}}\right) u_{\mathrm{F}}=Q_{\mathrm{R}} u_{\mathrm{D}}-Q_{\mathrm{L}} u_{\mathrm{E}}
$$

is satisfied with $u_{\mathrm{E}}=0$, or, equivalently, and since we consider Model 1 only,

$$
u_{\mathrm{F}}\left(q_{\mathrm{R}}-q_{\mathrm{L}}\right) / q_{\mathrm{R}}=u_{\mathrm{D}} .
$$

These steady states represent either the conventional or the high-rate mode of continuous operation shown in Figure 1 (a) and Figure 1 (b), respectively.

At this point it should be emphasized that our steady-state problem is in general overdetermined. In fact, fixing $u_{\mathrm{D}}$ and integrating (5.1) upwards and obeying entropy and jump conditions, we will in general not achieve a solution with $\left.u\right|_{x<x_{\mathrm{L}}}=u_{\mathrm{E}}=0$. All profiles with $\left.u\right|_{w<x_{\mathrm{L}}} \neq u_{\mathrm{E}}=0$ have to be rejected as candidates for steady-state entropy solutions, since the global mass balance (5.10) is a consequence of the weak formulation (5.2). To make the analysis transparent, we will in some instances write out the symbol $u_{\mathrm{E}}$ in manipulations before setting it to zero. One result of this procedure is that under our model assumptions, no steady states with the compression region completely contained in the thickening zone but with a non-zero effluent concentration exist.

To determine a steady-state entropy weak solution that satisfies the global mass balance, it is in general necessary, say, to fix $u_{\mathrm{F}}$, choose $u_{\mathrm{D}}$, solve (5.1), to verify whether (5.10) is satisfied with $u_{\mathrm{E}}$ replaced by $u\left(x_{\mathrm{L}}^{-}\right)$, and to iterate this solution procedure (for example, by varying $u_{\mathrm{D}}$ ) until the global mass balance (5.10) is attained. However, under the simplifying assumption (5.9), it turns out that solutions with $u_{\mathrm{E}}=0$ can easily be characterized: these are those steady-state entropy weak solutions for which the compression region is strictly contained in the container, i.e., for which $\inf \left\{x \in \mathbb{R}: u(x)>u_{\mathrm{c}}\right\}>x_{\mathrm{L}}$. This is the most important subclass of steady states, since they are the most desired mode of operation (see Figure 1). Moreover, it turns out that these steady-state entropy weak solutions are strictly increasing.

5.1. Steady-state solution in the discharge zone. Before proceeding to integrate the ODE (5.1) upwards from $x=x_{\mathrm{R}}$, we consider the discharge zone $x>x_{\mathrm{R}}$. Since we are seeking solutions for which for which $A(v)$ is continuous, we conclude that $A\left(u\left(x_{\mathrm{R}}^{+}\right)\right)=A\left(u\left(x_{\mathrm{R}}^{-}\right)\right)=A\left(u_{\mathrm{D}}\right)$ and therefore $u\left(x_{\mathrm{R}}^{+}\right)=u_{\mathrm{D}}$. On the other hand, from (5.1) we infer that the steady-state solution must be constant for $x>x_{\mathrm{R}}$. We conclude that $u(x)=u_{\mathrm{D}}$ for $x>x_{\mathrm{R}}$.

5.2. Steady-state solution in the thickening zone. Now that the steady-state solution has been determined in the interval $\left(x_{\mathrm{R}}, \infty\right)$, we determine the solution in the interval $\left(0, x_{\mathrm{R}}\right)$. To this end, note first that as a consequence of the jump condition (5.4), the steady state solution must satisfy the condition $q_{\mathrm{R}} u_{\mathrm{D}}+b\left(u_{\mathrm{D}}\right)-\left.A(u)^{\prime}\right|_{x=x_{\mathrm{R}}^{+}}=q_{\mathrm{R}} u_{\mathrm{D}}$, which means

$$
b\left(u_{\mathrm{D}}\right)-\left.A(u)^{\prime}\right|_{x=x_{\mathrm{R}}^{+}}=0 .
$$

Assume now that $v(x)$ is a continuously differentiable solution of the following one-sided boundary value problem, which is the subcase of $(5.1)$ occurring for the interval $\left(0, x_{\mathrm{R}}\right]$ :

$$
q_{\mathrm{R}}\left(u-u_{\mathrm{D}}\right)+b(u)-A(u)^{\prime}=0 \quad \text { for } x<x_{\mathrm{R}}, \quad u\left(x_{\mathrm{R}}\right)=u_{\mathrm{D}} .
$$


Note that we have used (5.12) to reduce the second-order ODE (5.1) to the first-order ODE (5.13). We consider the solution of (5.13) on the interval $\left[x_{\mathrm{c}}, x_{\mathrm{R}}\right]$, where

$$
x_{\mathrm{c}}:=\inf \left\{x \in\left(0, x_{\mathrm{R}}\right] \mid u(x) \text { is the solution of (5.13) and } u(x)>u_{\mathrm{c}}\right\} .
$$

However, not every solution of (5.13) is an acceptable steady state solution. Rather, the following lemma shows that the entropy condition (5.3) imposes an additional admissibility condition. This condition imposes a restriction on the choice of $q_{\mathrm{R}}$ and $u_{\mathrm{D}}$ for a given flux density function $b(u)$.

Lemma 5.3. Any steady-state entropy solution $u(x)$ of the one-sided boundary value problem (5.13) on the interval $\left(x_{\mathrm{c}}, x_{\mathrm{R}}\right)$ is monotonically increasing, i.e. $u^{\prime}(x) \geq 0$ for $x \in\left[x_{\mathrm{c}}, x_{\mathrm{R}}\right]$. This statement is equivalent to the requirement

$$
q_{\mathrm{R}} u_{\mathrm{D}} \leq q_{\mathrm{R}} k+b(k) \text { for all } k \text { between } u(x) \text { and } u_{\mathrm{D}} \text { for } x \in\left[x_{\mathrm{c}}, x_{\mathrm{R}}\right] .
$$

Proof. In view of (5.12), we obtain from (5.5) the inequality

$$
\begin{aligned}
& \forall k \in \mathbb{R}: \forall x \in\left(x_{\mathrm{c}}, x_{\mathrm{R}}\right): \quad \operatorname{sgn}\left(u_{\mathrm{D}}-k\right)\left(q_{\mathrm{R}}\left(u_{\mathrm{D}}-k\right)-b(k)\right) \\
& -\operatorname{sgn}(u(x)-k)\left[q_{\mathrm{R}}(u(x)-k)+b(u(x))-b(k)-A(u)^{\prime}\right] \leq 0 .
\end{aligned}
$$

We now fix $x \in\left(x_{\mathrm{c}}, x_{\mathrm{R}}\right)$ and evaluate (5.16) for different values of $k$. Setting $k<\min \left\{u(x), u_{\mathrm{D}}\right\}$ and $k>\max \left\{u(x), u_{\mathrm{D}}\right\}$, we obtain $\pm\left[q_{\mathrm{D}} u_{\mathrm{D}}-q_{\mathrm{R}} u-b(u)+A(u)^{\prime}\right] \leq 0$, which in view of (5.13) is no new information. The choices $k=u(x)$ and $k=u_{\mathrm{D}}$ are covered as limiting cases in the subsequent discussion of the two alternatives in which $k$ is located strictly between $u(x)$ and $u_{\mathrm{D}}$.

Assume first that $u_{\mathrm{D}}<k<u(x)$. Then (5.16) leads to the inequality

$$
2\left(q_{\mathrm{R}} k+b(k)\right)-q_{\mathrm{R}} u_{\mathrm{D}}-q_{\mathrm{R}} u(x)-b(u(x))+A(u)^{\prime} \leq 0 \quad \text { for all } k \in\left(u_{\mathrm{D}}, u(x)\right) .
$$

Using that $-q_{\mathrm{R}} u(x)-b(u(x))+A(u)^{\prime}=-q_{\mathrm{R}} u_{\mathrm{D}}$, we obtain from (5.17)

$$
q_{\mathrm{R}}\left(k-u_{\mathrm{D}}\right)+b(k) \leq 0 \text { for all } k \in\left(u_{\mathrm{D}}, u(x)\right) .
$$

However, (5.18) can never be satisfied, since $q_{\mathrm{R}}>0, k>u_{\mathrm{D}}$, and we assume $b \geq 0$.

The remaining case is the assumption $u(x)<k<u_{\mathrm{D}}$, which leads to the inequality

$$
-2\left(q_{\mathrm{R}} k+b(k)\right)+q_{\mathrm{R}} u_{\mathrm{D}}+q_{\mathrm{R}} u(x)+b(u(x))-A(u)^{\prime} \leq 0 \text { for all } k \in\left(u(x), u_{\mathrm{D}}\right) .
$$

Using that $q_{\mathrm{R}} u(x)+b(u(x))-A(u)^{\prime}=q_{\mathrm{R}} u_{\mathrm{D}}$ and that $b\left(u_{\mathrm{D}}\right)>0$, we obtain from $(5.19)$

$$
q_{\mathrm{R}} u_{\mathrm{D}} \leq q_{\mathrm{R}} k+b(k) \text { for all } k \in\left[u(x), u_{\mathrm{D}}\right] .
$$

Since (5.13) can be rearranged to give

$$
u^{\prime}(x)=\frac{q_{\mathrm{R}}\left(u(x)-u_{\mathrm{D}}\right)+b(u(x))}{a(u(x))},
$$

we see that (5.20) implies $u^{\prime}(x) \geq 0$ for $x \in\left[x_{\mathrm{c}}, x_{\mathrm{R}}\right]$.

Remark 5.2. Note that (5.20) has a useful graphical interpretation: namely, the graph of $g_{\mathrm{R}}(u)=$ $q_{\mathrm{R}} u+b(u)$ must lie above the horizontal line $f=q_{\mathrm{R}} u_{\mathrm{D}}$ fixed by the desired operation data. This condition implies a limitation of the attainable solids throughput for given material and vessel.

To proceed with the discussion, we distinguish between three cases: $x_{\mathrm{c}}>0$ (Case 1 ), $x_{\mathrm{c}}=0$ and $u\left(0^{+}\right)>u_{\mathrm{c}}($ Case 2$)$, and $x_{\mathrm{c}}=0$ and $u\left(0^{+}\right)=u_{\mathrm{c}}$ (Case 3$)$.

Case $1\left(x_{\mathrm{c}}>0\right)$. The Rankine-Hugoniot and entropy jump conditions across $x=x_{\mathrm{c}}$ are

$$
\begin{aligned}
q_{\mathrm{R}} u\left(x_{\mathrm{c}}^{-}\right) & +b\left(u\left(x_{\mathrm{c}}^{-}\right)\right)-\left.A(u)^{\prime}\right|_{x=x_{\mathrm{c}}^{-}}=q_{\mathrm{R}} u\left(x_{\mathrm{c}}^{+}\right)+b\left(u\left(x_{\mathrm{c}}^{+}\right)\right)-\left.A(u)^{\prime}\right|_{x=x_{\mathrm{c}}^{+}}, \\
\forall k \in \mathbb{R}: & \operatorname{sgn}\left(u\left(x_{\mathrm{c}}^{+}\right)-k\right)\left[q_{\mathrm{R}}\left(u\left(x_{\mathrm{c}}^{+}\right)-k\right)+b\left(u\left(x_{\mathrm{c}}^{+}\right)\right)-b(k)-\left.A(u)^{\prime}\right|_{x=x_{\mathrm{c}}^{+}}\right] \\
- & \operatorname{sgn}\left(u\left(x_{\mathrm{c}}^{-}\right)-k\right)\left[q_{\mathrm{R}}\left(u\left(x_{\mathrm{c}}^{-}\right)-k\right)+b\left(u\left(x_{\mathrm{c}}^{-}\right)\right)-b(k)-\left.A(u)^{\prime}\right|_{x=x_{\mathrm{c}}^{-}}\right] \leq 0,
\end{aligned}
$$

respectively. Moreover, from Lemma 5.2 it follows that $A\left(u\left(x_{\mathrm{c}}^{-}\right)\right)=A\left(u\left(x_{\mathrm{c}}^{+}\right)\right)=A\left(u_{\mathrm{c}}\right)=0$, so that $0 \leq u\left(x_{\mathrm{c}}^{-}\right) \leq u_{\mathrm{c}}$. From (5.13) and the definition of $x_{\mathrm{c}}$ it follows that $q_{\mathrm{R}} u\left(x_{\mathrm{c}}^{+}\right)+b\left(u\left(x_{\mathrm{c}}^{+}\right)\right)-$ $\left.A(u)^{\prime}\right|_{x=x_{\mathrm{c}}^{+}}=q_{\mathrm{R}} u_{\mathrm{D}}$. Inserting this into (5.22), we get

$$
q_{\mathrm{R}} u\left(x_{\mathrm{c}}^{-}\right)+b\left(u\left(x_{\mathrm{c}}^{-}\right)\right)-\left.A(u)^{\prime}\right|_{x=x_{\mathrm{c}}^{-}}=q_{\mathrm{R}} u_{\mathrm{D}} .
$$


Inserting (5.13), (5.24) and $u\left(x_{\mathrm{c}}^{+}\right)=u_{\mathrm{c}}$ into (5.23) yields

$$
\forall k \in \mathbb{R}: \quad \operatorname{sgn}\left(u_{\mathrm{c}}-k\right)\left(q_{\mathrm{R}} u_{\mathrm{D}}-q_{\mathrm{R}} k-b(k)\right)-\operatorname{sgn}\left(u\left(x_{\mathrm{c}}^{-}\right)-k\right)\left(q_{\mathrm{R}} u_{\mathrm{D}}-q_{\mathrm{R}} k-b(k)\right) \leq 0 .
$$

Obviously, the unique non-trivial case that needs to be discussed here is $u\left(x_{\mathrm{c}}^{-}\right)<k<u_{\mathrm{c}}$. Then we have $\operatorname{sgn}\left(u_{\mathrm{c}}-k\right)=1, \operatorname{sgn}\left(u\left(x_{\mathrm{c}}^{-}\right)-k\right)=-1$, and the inequality is reduced to

$$
q_{\mathrm{R}} u_{\mathrm{D}} \leq g_{\mathrm{R}}(k)=q_{\mathrm{R}} k+b(k) \text { for all } k \in\left(u\left(x_{\mathrm{c}}^{-}\right), u_{\mathrm{c}}\right) .
$$

On the other hand, from (5.15) we infer that $q_{\mathrm{R}} u_{\mathrm{D}} \leq q_{\mathrm{R}} u_{\mathrm{c}}+b\left(u_{\mathrm{c}}\right)$. This means that at $u=u_{\mathrm{c}}$, the graph of $g_{\mathrm{R}}(u)$ lies above or intersects the horizontal line $f=q_{\mathrm{R}} u_{\mathrm{D}}$. Consequently, $u\left(x_{\mathrm{c}}^{-}\right)$is the largest intersection of $g_{\mathrm{R}}(u)$ with the horizontal line $f=q_{\mathrm{R}} u_{\mathrm{D}}$ that is smaller or equal to $u_{\mathrm{c}}$ :

$$
u\left(x_{\mathrm{c}}^{-}\right)=\inf \left\{u \in\left[0, u_{\mathrm{c}}\right] \mid \forall \xi \in\left[u, u_{\mathrm{c}}\right]: g_{\mathrm{R}}(\xi)=q_{\mathrm{R}} \xi+b(\xi) \geq q_{\mathrm{R}} u_{\mathrm{D}}\right\} .
$$

Since $g_{\mathrm{R}}(0)=0<q_{\mathrm{R}} u_{\mathrm{D}}$ and $g_{\mathrm{R}}\left(u_{\mathrm{c}}\right)>q_{\mathrm{R}} u_{\mathrm{D}}$ by assumption, it is ensured that the curve $u \mapsto g_{\mathrm{R}}(u)$ and the horizontal line $u \mapsto q_{\mathrm{R}} u_{\mathrm{D}}$ always intersect on $\left[0, u_{\mathrm{c}}\right]$, and thus $u\left(x_{\mathrm{c}}^{-}\right)$is well defined. Note that for a function $b(u)$ with exactly one inflection point, the infimum is taken over at most three solutions of the equation $q_{\mathrm{R}} u+b(u)=q_{\mathrm{R}} u_{\mathrm{D}}$. It is not difficult to see that the steady-state solution in the interval $\left(x_{\mathrm{c}}, 0\right)$ is given by the constant $u\left(x_{\mathrm{c}}^{-}\right)$, which is uniquely constructed here.

It is at this point that assumption (5.9) turns out to be convenient in order to reduce the number of possible cases occurring in the continuation of the solution into the clarification zone. There would be no difficulty associated with relaxing this assumption.

Cases 2 and $3\left(x_{\mathrm{c}}=0, v\left(0^{+}\right) \geq u_{\mathrm{c}}\right)$ The construction of the steady-state solution in the thickening zone $\left(0, x_{\mathrm{R}}\right]$ is completed. The differentiable solution profile is given by the solution of the one-sided boundary value problem (5.13).

\subsection{Steady-state solution in the clarification zone.}

Case $1\left(x_{\mathrm{c}}>0\right)$. At $x=0$, the next flux discontinuity has to be dealt with. However, since the solution for $x>0$ is a constant not exceeding $u_{\mathrm{c}}$ and since $A(u)$ is continuous across $x=0$, we have to treat a transition between two fluxes of a hyperbolic conservation law. The entropy weak solution to this problem has been determined in several papers [19, 39, 40,41, 46, 47, 48, 58, 75]. The basic complication is that if the fluxes adjacent to $x=0$ are non-monotone, then there might be several possibilities to satisfy the Rankine-Hugoniot condition if $u\left(0^{+}\right)$is given, and an entropy condition is necessary to single out the unique entropy-satisfying solution. This will in general generate a multitude of cases here, depending on the flux parameters, properties of the function $b$, and on which solution of the equation $q_{\mathrm{R}} u+b(u)=q_{\mathrm{D}} u_{\mathrm{D}}$ yields the relevant state $u\left(0^{+}\right)$.

All these cases can be handled by the recent theory of conservation laws with discontinuous flux. However, assumption (5.9) helps to avoid this complication since it ensures that to a given value $u\left(0^{+}\right)$there corresponds a unique value $u\left(0^{-}\right)$such that the jump condition across $x=0$,

$$
q_{\mathrm{R}}\left(u\left(0^{+}\right)-u_{\mathrm{F}}\right)+b\left(u\left(0^{+}\right)\right)=q_{\mathrm{L}}\left(u\left(0^{-}\right)-u_{\mathrm{F}}\right)+b\left(u\left(0^{-}\right)\right)
$$

is satisfied. To see this, recall that the constancy of $u$ on $\left(0, x_{\mathrm{c}}\right)$ and (5.24) imply $q_{\mathrm{R}} u\left(0^{+}\right)+$ $b\left(u\left(0^{+}\right)\right)=q_{\mathrm{R}} u_{\mathrm{D}}$. Inserting this into (5.26), we get

$$
-\left(q_{\mathrm{R}}-q_{\mathrm{L}}\right) u_{\mathrm{F}}+q_{\mathrm{R}} u_{\mathrm{D}}=q_{\mathrm{L}} u\left(0^{-}\right)+b\left(u\left(0^{-}\right)\right) .
$$

However, the left-hand side of (5.27) is just $q_{\mathrm{L}} u_{\mathrm{E}} \leq 0$. On the other hand, due to (5.9) and since we seek a solution $0 \leq u\left(0^{-}\right) \leq u_{\mathrm{c}}$, the right-hand part of (5.27) is nonnegative, and is zero only for $u\left(0^{-}\right)=0$. Thus, the only solution is $u_{\mathrm{E}}=0, u\left(0^{-}\right)=0$, which implies $u(x)=0$ for all $x \geq 0$.

Consequently, under the assumption (5.9) the only steady-state entropy weak solutions for which the sediment level $x=x_{\mathrm{c}}$ is located strictly below the feed level $x=0$ are solutions for which there is only clear liquid in the clarification and overflow zones $(x \geq 0)$. For these solutions, the feed and discharge concentrations $u_{\mathrm{F}}$ and $u_{\mathrm{D}}$ are linked by $\left(q_{\mathrm{R}}-q_{\mathrm{L}}\right) u_{\mathrm{F}}=q_{\mathrm{R}} u_{\mathrm{D}}$.

Case $2\left(x_{\mathrm{c}}=0, u\left(0^{+}\right)>u_{\mathrm{c}}\right)$. Lemma 5.2 implies that $u\left(0^{-}\right)=u\left(0^{+}\right)$if $u^{+}>u_{\mathrm{c}}$. Thus, we can continue to solve (5.1) in the clarification zone $x \in\left(x_{\mathrm{L}}, 0\right)$. Integrating this ODE over the interval $(x, 0)$, we obtain the following one-sided boundary value problem for a first-order ODE:

$$
q_{\mathrm{L}}\left(u(x)-u\left(0^{-}\right)\right)+b(u)-b\left(u\left(0^{-}\right)\right)-\left.A(u)^{\prime}\right|_{x=0^{-}}=0 \quad \text { for } x<0, \quad u(0)=u\left(0^{-}\right) .
$$


To determine $u^{\prime}\left(0^{-}\right)$, we use the Rankine-Hugoniot condition (5.4) across $x=0$,

$$
q_{\mathrm{L}}\left(u\left(0^{-}\right)-u_{\mathrm{F}}\right)+b\left(u\left(0^{-}\right)\right)-\left.A(u)^{\prime}\right|_{x=0^{-}}=q_{\mathrm{R}}\left(u\left(0^{+}\right)-u_{\mathrm{F}}\right)+b\left(u\left(0^{+}\right)\right)-\left.A(u)^{\prime}\right|_{x=0^{+}} .
$$

Recalling that we already know that $u\left(0^{-}\right)=u\left(0^{+}\right)$, we get

$$
\left.A(u)^{\prime}\right|_{x=0^{-}}=\left(q_{\mathrm{L}}-q_{\mathrm{R}}\right)\left(u\left(0^{-}\right)-u_{\mathrm{F}}\right)+\left.A(u)^{\prime}\right|_{x=0^{+}} .
$$

On the other hand, in the present case we know that $\left.A(u)^{\prime}\right|_{x=0^{+}}=q_{\mathrm{R}}\left(u\left(0^{+}\right)-u_{\mathrm{D}}\right)+b\left(u\left(0^{+}\right)\right)$. Inserting this into (5.30) and replacing $u\left(0^{+}\right)$by $u\left(0^{-}\right)$, we get

$$
\left.A(u)^{\prime}\right|_{x=0^{-}}=q_{\mathrm{L}} u\left(0^{-}\right)-q_{\mathrm{R}} u_{\mathrm{D}}-\left(q_{\mathrm{L}}-q_{\mathrm{R}}\right) u_{\mathrm{F}}+b\left(u\left(0^{-}\right)\right) .
$$

Finally, we insert (5.31) into (5.28), and obtain the one-sided boundary value problem

$$
q_{\mathrm{L}} u(x)+b(u)-A(u)^{\prime}-q_{\mathrm{R}} u_{\mathrm{D}}-\left(q_{\mathrm{L}}-q_{\mathrm{R}}\right) u_{\mathrm{F}}=0, \quad x<0 ; \quad v(0)=v\left(0^{-}\right) .
$$

We now define

$$
\tilde{x}_{\mathrm{c}}:=\inf \left\{x \in\left[x_{\mathrm{L}}, 0\right) \mid u(x) \text { is the solution of (5.28) and } u_{\max } \geq u(x)>u_{\mathrm{c}}\right\}
$$

and recall from (5.32) and (5.31) that we have

$$
b(u(x))-A(u)^{\prime}=-q_{\mathrm{L}} u(x)+q_{\mathrm{R}} u_{\mathrm{D}}+\left(q_{\mathrm{L}}-q_{\mathrm{R}}\right) u_{\mathrm{F}} \quad \text { for } x \in\left(\tilde{x}_{\mathrm{c}}, 0\right],
$$

as well as that we obtain from (5.5) the inequality

$$
\left[\operatorname{sgn}\left(u\left(0^{-}\right)-k\right)-\operatorname{sgn}(u(x)-k)\right]\left(-q_{\mathrm{L}} k-b(k)+q_{\mathrm{R}} u_{\mathrm{D}}+\left(q_{\mathrm{L}}-q_{\mathrm{R}}\right) u_{\mathrm{F}}\right) \leq 0
$$

for all $x \in\left(\tilde{x}_{\mathrm{c}}, 0\right)$ and for all $k \in \mathbb{R}$.

We observe that $q_{\mathrm{R}} u_{\mathrm{D}}+\left(q_{\mathrm{L}}-q_{\mathrm{R}}\right) u_{\mathrm{F}}=q_{\mathrm{L}} u_{\mathrm{E}}$. Then, the solution in the interval $\left(\tilde{x}_{\mathrm{c}}, 0\right)$ is given by the solution of the one-sided boundary problem (which is a slight rearrangement of (5.32))

$$
u^{\prime}(x)=\frac{q_{\mathrm{L}} u(x)+b(u(x))-q_{\mathrm{L}} u_{\mathrm{E}}}{a(u(x))}, \quad x<0, \quad u(0)=u\left(0^{-}\right) .
$$

Due to our assumption (5.9), since $g_{\mathrm{L}}(0)=0$, and $b(u)$ and therefore $g_{\mathrm{L}}(u)$ has exactly one inflection point, we know that $g_{\mathrm{L}}(u)$ has exactly one positive maximum $u_{\mathrm{M}}>u_{\mathrm{c}}$, and that $g_{\mathrm{L}}(u)$ is monotonically decreasing between $u_{\mathrm{M}}$ and $u_{\max }=1$ with $g_{\mathrm{L}}\left(u_{\max }\right)=q_{\mathrm{L}} u_{\max }<0$. Consequently, there exists exactly one point $u^{*}$ with $u_{\mathrm{c}}<u^{*}<u_{\max }$ such that $q_{\mathrm{L}} u^{*}+b\left(u^{*}\right)=q_{\mathrm{L}} u_{\mathrm{E}}$. Since the maximum of $g_{\mathrm{L}}$ is positive but $q_{\mathrm{L}} u_{\mathrm{E}} \leq 0$, we know that $u^{*}>u_{\mathrm{M}}$ and therefore

$$
g_{\mathrm{L}}(u)<g_{\mathrm{L}}\left(u^{*}\right) \text { for } u>u^{*}, u \leq u_{\max } .
$$

We first assume that $u\left(0^{+}\right)=u\left(0^{-}\right)>u^{*}$. Our immediate goal is to show that $u\left(0^{-}\right)>u^{*}$ does not lead to an admissible steady-state solution. Note that by the discussion of the solution in the thickening zone, we know that $u\left(0^{-}\right)<u_{\mathrm{D}}$.

By the definition of $u^{*}$, we may rewrite the ODE (5.35) as

$$
u^{\prime}(x)=\frac{g_{\mathrm{L}}(u(x))-g_{\mathrm{L}}\left(u^{*}\right)}{a(u(x))}, \quad x<0, \quad u(0)=u\left(0^{-}\right) .
$$

In light of (5.36), we see that inserting $u\left(0^{-}\right)>u^{*}$ will cause the right-hand part of the ODE in (5.35) to be negative at $x=0$, and this right-hand part remains negative if we proceed with the integration of (5.35), since we produce a solution that is monotonically decreasing (increasing upwards). This integration may be continued upwards until either $u=u_{\max }=1$ is attained or $x=x_{\mathrm{L}}$ is reached. In the first case, however, there is no valid way to continue the solution to the remaining interval $\left(x_{\mathrm{L}}, \tilde{x}_{\mathrm{c}}\right)$ other than setting $u=u_{\max }=1$ for $x \in\left(x_{\mathrm{L}}, \tilde{x}_{\mathrm{c}}\right)$. This means that at $x=x_{\mathrm{L}}$, the jump condition implies that $q_{\mathrm{L}} u_{\max }=q u_{\mathrm{E}}$, that is $u_{\mathrm{E}}=u_{\max }$, in contradiction to the assumption $u_{\mathrm{E}}<u_{\max }$. In the other case, in which $x=x_{\mathrm{L}}$ is reached by integrating (5.35), we have the following Rankine-Hugoniot condition across $x=x_{\mathrm{L}}$ :

$$
q_{\mathrm{L}} u\left(x_{\mathrm{L}}^{+}\right)+b\left(u\left(x_{\mathrm{L}}^{+}\right)\right)-\left.A(u)^{\prime}\right|_{w=x_{\mathrm{L}}^{+}}=q_{\mathrm{L}} u\left(x_{\mathrm{L}}^{-}\right) .
$$

On the other hand, since $u\left(x_{\mathrm{L}}^{+}\right) \geq u\left(0^{-}\right)>u_{\mathrm{c}}$, we have $A\left(u\left(x_{\mathrm{L}}^{+}\right)\right)>0$ and thus, due to Lemma 5.2 , $u\left(x_{\mathrm{L}}^{+}\right)=u\left(x_{\mathrm{L}}^{-}\right)$, and therefore (5.37) reduces to $b\left(u\left(x_{\mathrm{L}}^{+}\right)\right)=\left.A(u)^{\prime}\right|_{x=x_{\mathrm{L}}^{+}}$. However, since by assumption $u^{\prime}\left(x_{\mathrm{L}}^{+}\right)<0$, we have that $\left.A^{\prime}(u)\right|_{x=x_{\mathrm{L}}^{+}}<0$, which in turn implies $b\left(u\left(x_{\mathrm{L}}^{+}\right)\right)<0$. This 
is a contradiction to the nonnegativity of $b$. Thus, no admissible steady-state solution can be constructed if $u\left(0^{-}\right)>u^{*}$.

The case $u\left(0^{-}\right)=u^{*}$ equally leads to an inadmissible solution, since integrating (5.35) leads to the constant solution $u \equiv u^{*}$ on $\left(x_{\mathrm{L}}, 0\right)$. Similarly to the discussion of the previous case, the jump condition (5.37) and Lemma 5.2 imply $b\left(u^{*}\right)=\left.A(u)^{\prime}\right|_{x=x_{\mathrm{L}}^{+}}$. However, the constancy of $u$ along $x \in\left(x_{\mathrm{L}}, 0\right)$ implies $\left.A(u)^{\prime}\right|_{x=x_{\mathrm{L}}^{+}}=0$ and therefore $b\left(u^{*}\right)=0$, in contradiction to the assumed properties of $b$. Another reason to reject the profiles with $u\left(0^{-}\right) \geq u^{*}$ as candidates for steadystate entropy weak solutions is the violation of the global conservation principle (5.10), since we have chosen $u_{\mathrm{D}}$ and $u_{\mathrm{F}}$ such that $u_{\mathrm{E}}=0$, but in these cases our integration yields positive values of $u\left(x_{\mathrm{L}}^{-}\right)$, which should equal $u_{\mathrm{E}}$.

We now look at the remaining case $u\left(0^{-}\right)<u^{*}$. Then the right-hand side of the ODE in (5.35) is always positive, which implies a monotonically increasing (decreasing upwards) solution $u(x)$ until $x=\tilde{x}_{\mathrm{c}}$ is reached. This solution also satisfies the entropy condition. In fact, for any $x \in\left(\tilde{x}_{\mathrm{c}}, 0\right)$ with $u(x)<u\left(0^{-}\right)$and for all $k \in\left(u(x), u\left(0^{-}\right)\right.$), condition (5.34) (which is void for all other values of $k$ and for $\left.u(x)=u\left(0^{-}\right)\right)$implies $2\left(-q_{\mathrm{L}} k-b(k)+q_{\mathrm{L}} u_{\mathrm{E}}\right) \leq 0$, i.e.,

$$
q_{\mathrm{L}} k+b(k)-q_{\mathrm{L}} u_{\mathrm{E}} \geq 0 \quad \text { for all } k \in\left(u(x), u\left(0^{-}\right)\right),
$$

which in view of (5.35) is satisfied if $u(x)$ is a monotonically increasing solution on $\left(\tilde{x}_{\mathrm{c}}, 0\right)$.

We summarize our discussion of Case 2 in the clarification zone by the following lemma.

Lemma 5.4. Any admissible steady-state entropy solution $u=u(x)$ of Model 1 with $u\left(0^{-}\right)=$ $u\left(0^{+}\right)>u_{\mathrm{c}}$ must satisfy $u\left(0^{-}\right)<u^{*}$, where $u^{*}$ is the unique point in $\left(u_{\mathrm{c}}, u_{\max }\right)$ satisfying $g_{\mathrm{L}}\left(u^{*}\right) \equiv$ $q_{\mathrm{L}} u^{*}+b\left(u^{*}\right)=q_{\mathrm{L}} u_{\mathrm{E}}$. This solution is monotonically increasing on the interval $\left(\tilde{x}_{\mathrm{c}}, 0\right)$, where $\tilde{x}_{\mathrm{c}}$ is defined by (5.33).

Remark 5.3. The statement of Lemma 5.4 also has an obvious graphical interpretation. However, this condition requires knowledge of the value $u\left(0^{+}\right)=u\left(0^{-}\right)$. Thus, it can be evaluated only after the solution in the thickening zone has been determined. Furthermore, combining this finding with Lemma 5.3 for the thickening zone, we see that in any of the Cases 1, 2 or 3, the entropy condition and jump conditions enforce that $u^{\prime}(x) \geq 0$ in the compression region.

With the present discussion, we have constructed a steady-state solution up to $\tilde{x}_{\mathrm{c}}$, provided that $u(x)>u_{\mathrm{c}}$ in the thickening zone $x \in\left(0, x_{\mathrm{R}}\right)$. To finish the steady-state construction, let us first recall that for sake of brevity and being well aware of the incompleteness of the treatment in the present paper, we limit the discussion to those steady states for which $\tilde{x}_{\mathrm{c}}>x_{\mathrm{L}}$. In this case, there is a jump located at $x=\tilde{x}_{\mathrm{c}}$. We now seek the constant solution value $u=u\left(\tilde{x}_{\mathrm{c}}^{-}\right)$in the interval $\left(x_{\mathrm{L}}, \tilde{x}_{\mathrm{c}}\right)$. This value must satisfy $0 \leq u\left(\tilde{x}_{\mathrm{c}}^{-}\right) \leq u_{\mathrm{c}}$. From the Rankine-Hugoniot condition that follows from (5.4), $q_{\mathrm{L}} u\left(\tilde{x}_{\mathrm{c}}^{-}\right)+b\left(\tilde{x}_{\mathrm{c}}^{-}\right)=q_{\mathrm{L}} u_{\mathrm{c}}+b\left(u_{\mathrm{c}}\right)-\left.A(u)^{\prime}\right|_{x=\tilde{x}_{\mathrm{c}}^{+}}$, we see that the constant $u\left(\tilde{x}_{\mathrm{c}}^{-}\right)=$ $u_{\mathrm{c}}$ is not a solution. Consequently, we look for a constant $0 \leq u\left(\tilde{x}_{\mathrm{c}}^{-}\right)<u_{\mathrm{c}}$. To this end, note that the steady-state jump condition at $x=x_{\mathrm{L}}$ is $g_{\mathrm{L}}\left(u\left(x_{\mathrm{L}}^{+}\right)\right)=q_{\mathrm{L}} u\left(x_{\mathrm{L}}^{+}\right)+b\left(u\left(x_{\mathrm{L}}^{+}\right)\right)=q_{\mathrm{L}} u\left(x_{\mathrm{L}}^{-}\right)=q_{\mathrm{L}} u_{\mathrm{E}}$. Taking into account that $g_{\mathrm{L}}(u)$ is a non-negative monotonically increasing function on $\left[0, u_{\mathrm{c}}\right]$, while the right-hand side is a non-positive constant, we conclude (similar as in the discussion of Case 1) that $u_{\mathrm{E}}=0$ and $u\left(\tilde{x}_{\mathrm{c}}^{-}\right)=0$, i.e., the solution is zero on $\left(x_{\mathrm{L}}, \tilde{x}_{\mathrm{c}}\right)$.

Remark 5.4. The last result means that the mathematical model correctly describes the elimination of the hindered settling region in steady-state operation when the sediment level (where $u=u_{\mathrm{c}}$ ) is allowed to rise above the feed level, as drawn in Figure 1 (b). No particles are elutriated from the compression region into the overflow. This supports the physical explanation that above the feed level, the sediment bed acts as a filter medium for the portion of the feed flow that is directed into the clarification zone.

If we added a small amount of hydrodynamic diffusion and used a strictly positive diffusion coefficient $a(u)$ such that the resulting model were strictly parabolic, then there would be no upper limit for the integration of ODEs like (5.28), and under the preconditions of Case 2 discussed here, the quantity $\tilde{x}_{\mathrm{c}}$ defined by (5.33) would always assume the value $x_{\mathrm{L}}$ (corresponding to the overflow level) with $u\left(x_{\mathrm{L}}^{+}\right)>0$. In other words, there would be a small but positive volume fraction of solids 
in the overflow. Steady-state concentration profiles for a clarifier-thickener model with a strictly positive diffusion coefficient that illustrate this situation are plotted, for example, in [81].

Finally, we mention that Lev et al. [64] use a steady-state clarifier-thickener model without compression effects, but with a hydrodynamic diffusion term instead. A discussion of possible concentration extrema at steady state leads them to the conclusion that the concentration must increase downwards. Our analysis shows that by applying the entropy concept to the steady-state $O D E$, this characterization remains valid even when hydrodynamic diffusion vanishes.

Case $3\left(x_{\mathrm{c}}=0, u\left(0^{+}\right)=u_{\mathrm{c}}\right)$. In this case, we have $A\left(u\left(0^{+}\right)\right)=0$ and due to the continuity of $A(u)$ across $x=0, A\left(u\left(0^{-}\right)\right)=0$, which means $u\left(0^{-}\right) \in\left[0, u_{\mathrm{c}}\right]$. Since in the present case, it has been possible to integrate (5.13) up to $x=0^{+}$, we can replace the Rankine-Hugoniot condition across $x=0,(5.29)$, by $q_{\mathrm{L}} u\left(0^{-}\right)+b\left(u\left(0^{-}\right)\right)-\left.A(u)^{\prime}\right|_{x=0^{-}}=\left(q_{\mathrm{L}}-q_{\mathrm{R}}\right) u_{\mathrm{F}}+q_{\mathrm{R}} u_{\mathrm{D}}$. On the other hand, the following entropy jump condition follows by evaluating (5.5) for $x=0$ :

$$
\begin{aligned}
\forall k \in \mathbb{R}: \quad & \operatorname{sgn}\left(u\left(0^{+}\right)-k\right)\left[q_{\mathrm{R}}\left(u\left(0^{+}\right)-k\right)+b\left(u\left(0^{+}\right)\right)-b(k)-\left.A(u)^{\prime}\right|_{x=0^{+}}\right] \\
& -\operatorname{sgn}\left(u\left(0^{-}\right)-k\right)\left[q_{\mathrm{L}}\left(u\left(0^{-}\right)-k\right)+b\left(u\left(0^{-}\right)\right)-b(k)-\left.A(u)^{\prime}\right|_{x=0^{-}}\right] \\
& \leq\left|q_{\mathrm{R}}\left(k-u_{\mathrm{F}}\right)-q_{\mathrm{L}}\left(k-u_{\mathrm{F}}\right)\right| .
\end{aligned}
$$

Inserting (5.29), using once again (5.13) and that $u\left(0^{+}\right)=u_{\mathrm{c}}$, we get

$$
\begin{aligned}
\forall k \in \mathbb{R}: & \operatorname{sgn}\left(u_{\mathrm{c}}-k\right)\left(-q_{\mathrm{R}} k-b(k)+q_{\mathrm{R}} u_{\mathrm{D}}\right) \\
& -\operatorname{sgn}\left(u\left(0^{-}\right)-k\right)\left(-q_{\mathrm{L}} k-b(k)+\left(q_{\mathrm{L}}-q_{\mathrm{R}}\right) u_{\mathrm{F}}+q_{\mathrm{R}} u_{\mathrm{D}}\right) \leq\left|\left(q_{\mathrm{R}}-q_{\mathrm{L}}\right)\left(k-u_{\mathrm{F}}\right)\right| .
\end{aligned}
$$

Choosing $k=0$ and exploiting that $b(k)=0$, we get

$$
\operatorname{sgn}\left(u_{\mathrm{c}}\right) q_{\mathrm{R}} u_{\mathrm{D}}-\operatorname{sgn}\left(u\left(0^{-}\right)\right)\left(\left(q_{\mathrm{L}}-q_{\mathrm{R}}\right) u_{\mathrm{F}}+q_{\mathrm{R}} u_{\mathrm{D}}\right) \leq\left|\left(q_{\mathrm{R}}-q_{\mathrm{L}}\right)\left(-u_{\mathrm{F}}\right)\right|,
$$

which in view of $u_{\mathrm{c}}>0, u_{\mathrm{F}}>0$ and $q_{\mathrm{R}}-q_{\mathrm{L}} \geq 0$ implies

$$
q_{\mathrm{R}} u_{\mathrm{D}}+\operatorname{sgn}\left(u\left(0^{-}\right)\right)\left(q_{\mathrm{R}}-q_{\mathrm{L}}\right) u_{\mathrm{F}}+\operatorname{sgn}\left(v\left(0^{-}\right)\right) q_{\mathrm{R}} u_{\mathrm{D}} \leq\left(q_{\mathrm{R}}-q_{\mathrm{L}}\right) u_{\mathrm{F}} .
$$

If $\operatorname{sgn}\left(u\left(0^{-}\right)\right)=1$, then the left-hand side of (5.41) equals $2 q_{\mathrm{R}} u_{\mathrm{D}}+\left(q_{\mathrm{R}}-q_{\mathrm{L}}\right) u_{\mathrm{F}}$. Thus, inequality (5.41) cannot be satisfied. The unique remaining option is $\operatorname{sgn}\left(u\left(0^{-}\right)\right)=0$, i.e. $u\left(0^{-}\right)=0$. It is then easily seen that the solution for $x<0$, including also the section $x<x_{\mathrm{L}}$, vanishes identically. Thus, the solution of Case 3 is the limiting case of Cases 1 and 2 for $u\left(0^{+}\right) \rightarrow u_{\mathrm{c}}$.

Remark 5.5. The condition $u^{\prime}(x) \geq 0$ is in full agreement with engineering intuition, since one expects that in a clarifier-thickener operating properly at steady state, the concentration increases downwards. In fact, in several previous papers dealing with a simpler model of an ideal continuous thickener [13, 16], which basically consists only of the thickening zone of the model discussed here, the condition $u^{\prime}(x) \geq 0$ was postulated as a separate requirement for the determination of admissible steady states following just from this intuition, and the graphical condition (5.20) was derived by using this assumption in (5.21). We now clearly see that the natural requirement that a steady state should be an entropy weak solution implies this monotonicity property in the thickening zone, and it is therefore unnecessary to introduce it as an additional condition.

Observe that in contrast to our analysis of the thickening zone, we do not apply the entropy condition to construct the restrictions on the parameters (expressed by Lemma 5.4) in the clarification and overflow zones); rather, we exploit the jump conditions to establish these restrictions, and then check that the admissible solution satisfies the entropy condition.

5.4. Examples of steady states. Here and in the numerical examples, the flocculated suspension is characterized by the functions $b(u)$ and $\sigma_{\mathrm{e}}(u)$ given by (2.6) and (2.9), respectively, with $v_{\infty}=$ $10^{-4} \mathrm{~m} / \mathrm{s}, C=5, \sigma_{0}=1.0 \mathrm{~Pa}, u_{\mathrm{c}}=0.1$ and $k=6$. The remaining parameters are $\Delta \varrho=$ $1500 \mathrm{~kg} / \mathrm{m}^{3}$ and $g=9.81 \mathrm{~m} / \mathrm{s}^{2}$. These values are fairly realistic and are also used in $[14,16]$.

Moreover, we assume the bulk velocities $q_{\mathrm{R}}=2.5 \times 10^{-6} \mathrm{~m} / \mathrm{s}$ and $q_{\mathrm{L}}=-1.0 \times 10^{-5} \mathrm{~m} / \mathrm{s}$. Thus, we are interested in steady states for which $u_{\mathrm{D}}=u_{\mathrm{F}}\left(q_{\mathrm{R}}-q_{\mathrm{L}}\right) / q_{\mathrm{R}}=5 u_{\mathrm{F}}$, and these parameters have been chosen so that assumption (5.9) is satisfied. The relevant flux functions for the thickening and clarification zone, $g_{\mathrm{R}}(u)$ and $g_{\mathrm{L}}(u)$, are plotted in Figure 3 . 

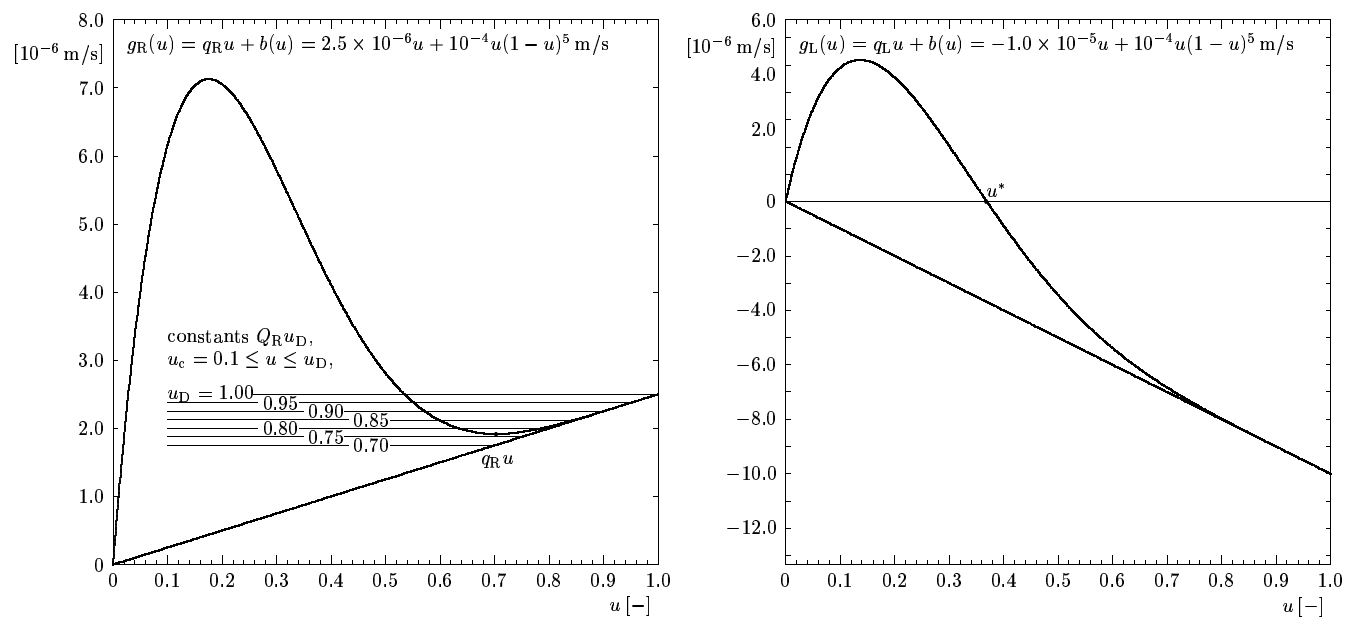

Figure 3. The flux functions $q_{\mathrm{R}} u+b(u)$ (left) and $q_{\mathrm{L}}+b(u)$ (right). The left diagram also shows the constant lines $q_{\mathrm{R}} u_{\mathrm{D}}$ for some values of $u_{\mathrm{D}}$.

We start the steady-state construction by fixing values of $u_{\mathrm{D}}$, and determine the corresponding value $x_{\mathrm{c}}$. We limit ourselves to those values $u_{\mathrm{D}}$ that ensure that the entropy condition (5.15) is satisfied. To this end, we consider the plot of $g_{\mathrm{R}}(u)$ and draw horizontal line segments $f=q_{\mathrm{R}} u_{\mathrm{D}}$ for a selection of values of $u_{\mathrm{D}}$ and for $u_{\mathrm{c}} \leq u \leq u_{\mathrm{D}}$, as has been done in the left plot of Figure 3 . We see that these lines remain strictly below the graph of $g_{\mathrm{R}}(u)$ for those values of $u_{\mathrm{D}}$ for which

$$
q_{\mathrm{R}} u_{\mathrm{D}}<\min _{u_{\mathrm{c}} \leq u \leq 1} g_{\mathrm{R}}(u)=g_{\mathrm{R}}(0.703) \approx 1.92 \times 10^{-6} \mathrm{~m} / \mathrm{s} .
$$

This implies that the entropy condition (5.15) is satisfied a priori (i.e., independently of the depth of the thickening zone $x_{\mathrm{R}}$ ) for all $u_{\mathrm{D}}$ with

$$
u_{\mathrm{c}} \leq u_{\mathrm{D}}<u_{\mathrm{D} \max }:=\frac{1}{q_{\mathrm{R}}} \min _{u_{\mathrm{c}} \leq u \leq 1} g_{\mathrm{R}}(u)=\frac{1.92 \times 10^{-6} \mathrm{~m} / \mathrm{s}}{2.5 \times 10^{-6} \mathrm{~m} / \mathrm{s}}=0.768 .
$$

For all other values of $u_{\mathrm{D}}$, it would be necessary to determine a solution to (5.13), and to check whether this is monotone on $\left[x_{\mathrm{c}}, x_{\mathrm{R}}\right]$. We will not pursue this here.

Given this limitation on $u_{\mathrm{D}}$, we choose the profiles for $u_{\mathrm{D}}=0.3,0.35,0.4,0.405,0.41, \ldots, 0.455$ for closer inspection. Solving (5.13) with a standard numerical ODE method, we obtain that for $u_{\mathrm{D}} \leq 0.41$, we have $x_{\mathrm{c}}>0$ and therefore steady states of Case 1 , while all other values lead to candidates for Case 2. Solving the equation $q_{\mathrm{R}} u\left(x_{\mathrm{c}}^{-}\right)+b\left(u\left(x_{\mathrm{c}}^{-}\right)\right)=q_{\mathrm{R}} u_{\mathrm{D}}$ numerically yields the following values of $u\left(x_{\mathrm{c}}^{-}\right)$, which are the constant values each entropy weak solution assumes on $\left[0, x_{\mathrm{c}}\right]$ :

\begin{tabular}{|c|ccccc|}
\hline$u_{\mathrm{D}}$ & 0.3 & 0.35 & 0.4 & 0.405 & 0.41 \\
\hline$u\left(x_{\mathrm{c}}^{-}\right)$ & 0.00759 & 0.00892 & 0.01026 & 0.01039 & 0.01053 \\
\hline
\end{tabular}

For these values of $u_{\mathrm{D}}$, the steady-state entropy weak solution in the clarification and overflow zones is zero. Figure 4 includes plots of these profiles.

It remains to deal with $u_{\mathrm{D}}=0.415,0.42, \ldots, 0.455$, the candidates for Case 2 , for which the clarification zone has to be examined. We have just found out that all of these concentration values admit an entropy-satisfying steady-state solution in the thickening zone. However, with the present parameters we have $u_{\mathrm{E}}=0$, which implies $u^{*}=0.369$. This point is marked in Figures 3 and 4. We see that integrating (5.13) from $u_{\mathrm{D}}=0.455$ leads to a profile with $u\left(0^{+}\right)>u^{*}$, which does not lead to an admissible solution in the clarification zone. The tentative profile for this case is the rightmost dotted profile in Figure 4 . For $u_{\mathrm{D}}=0.445$ and $u_{\mathrm{D}}=0.45$, we obtain admissible profiles in the clarification zone, which, however, reach the overflow level $x=x_{\mathrm{L}}$, and will produce an effluent with $u_{\mathrm{E}}>0$. These profiles are no admissible entropy steady-state solutions since the 


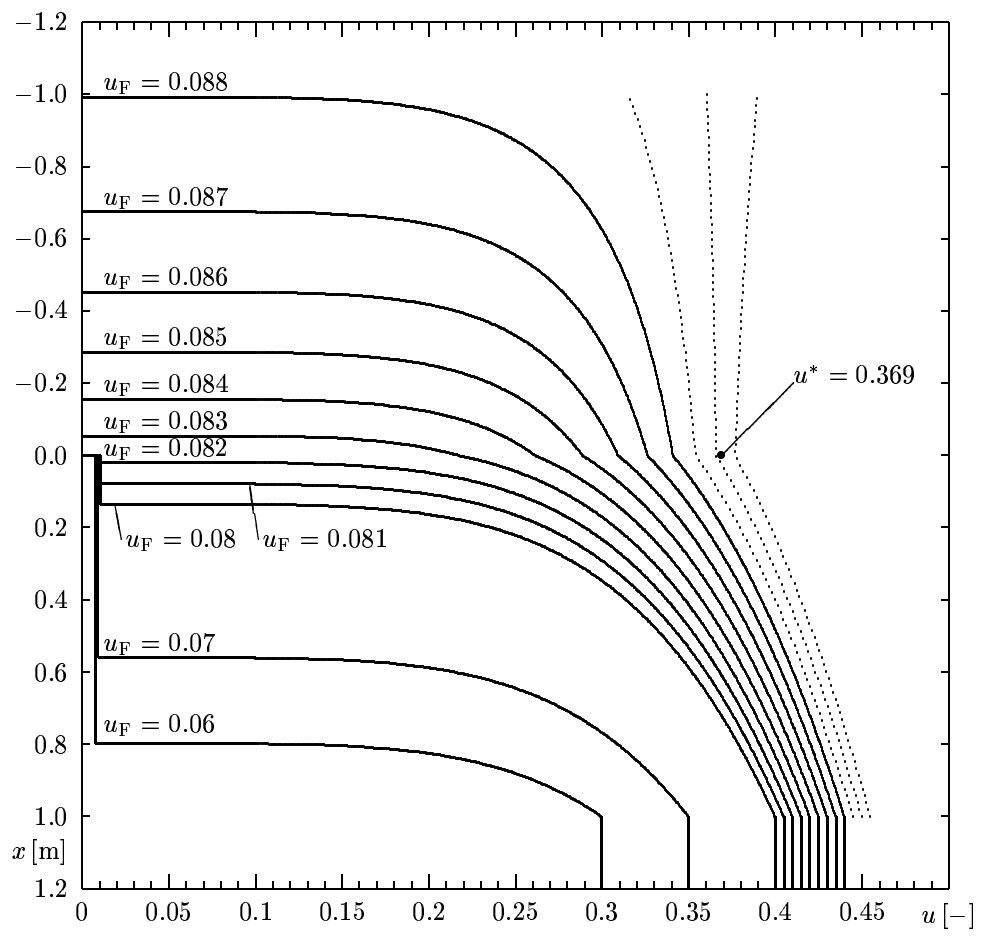

Figure 4. Steady-state concentration profiles in Vessel 1. The dotted curves show solutions of (5.13) and (5.28) that do not lead to admissible steady states with zero overflow concentration.

global conservation principle is violated. The values $u_{\mathrm{D}}=0.415,0.42, \ldots, 0.435$ lead to admissible steady-state profiles with $\tilde{x}_{\mathrm{c}}>x_{\mathrm{L}}$, and, as a consequence of our analysis, $u_{\mathrm{E}}=0$.

\section{NumERICAL EXAMPLES}

6.1. Preliminary remarks. Note that for $k \in \mathbb{N}$, as chosen here, standard calculus yields that the function $A(u)$ has the explicit representation $A(u)=\mathrm{A}(u)-\mathrm{A}\left(u_{\mathrm{c}}\right)$ for $u>u_{\mathrm{c}}$ with

$$
\mathrm{A}(u):=-\frac{v_{\infty} \sigma_{0}}{\Delta \varrho g u_{\mathrm{c}}^{k}}(1-u)^{C} u^{k} \sum_{j=1}^{k} c_{j}\left(\frac{1}{u}-1\right)^{j}, \quad c_{j}=\prod_{l=1}^{j} \frac{k+1-l}{C+l}, \quad j=1, \ldots, k ;
$$

it is straightforward to verify by differentiating $\mathrm{A}(u)$ that

$$
\frac{d \mathrm{~A}(u)}{d u}=v_{\infty} u(1-u)^{C} \cdot \frac{1}{\Delta \varrho g u} \cdot \frac{d}{d u}\left(\sigma_{0}\left(\left(u / u_{\mathrm{c}}\right)^{k}-1\right)\right)=\frac{v_{\infty} \sigma_{0}}{\Delta \varrho g u_{\mathrm{c}}^{k}}(1-u)^{C} k u^{k-1},
$$

so that the function $A(u)$ defined here indeed satisfies (2.10).

We consider two units, a cylindrical one (Vessel 1) and one with discontinuously varying crosssectional area (Vessel 2), see Figure 5. The interior of Vessel 1 is $S_{\text {int }}=1 \mathrm{~m}^{2}$ (recall that the outer pipe diameter $S_{0}$ can always be transformed away). The piecewise constant cross-sectional area function $S(x)$ of the non-cylindrical one, Vessel 2, is defined in Figure 5.

The motivation of the choice of $S(x)$ for Vessel 2 is that most clarifier-thickeners have a conically shaped bottom to facilitate transport of material to the discharge outlet, and that we assume that one quarter of the cross-sectional area in the clarification zone is occupied by installations related to the feed mechanisms. Observe that in the second case, both parameters $\gamma_{1}$ and $\gamma_{2}$ accounting for the cross-sectional area and the bulk flow, respectively, have a discontinuity at $x=0$. 

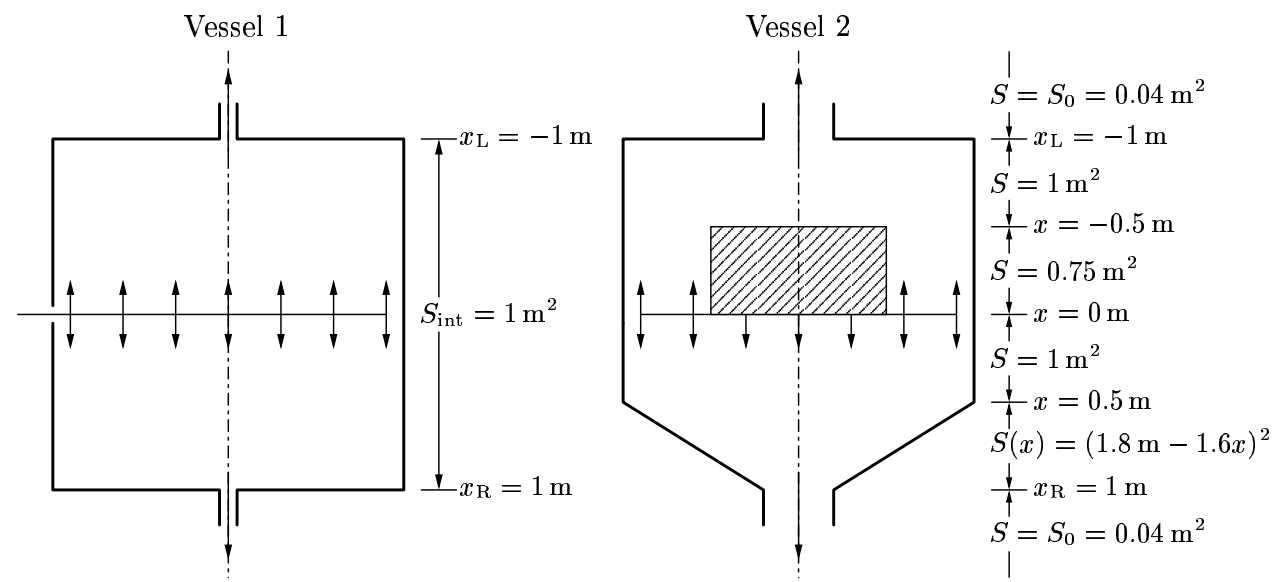

Figure 5. The cylindrical clarifier-thickener (left) and the unit with discontinuously varying cross-sectional area (right) used for numerical simulations.
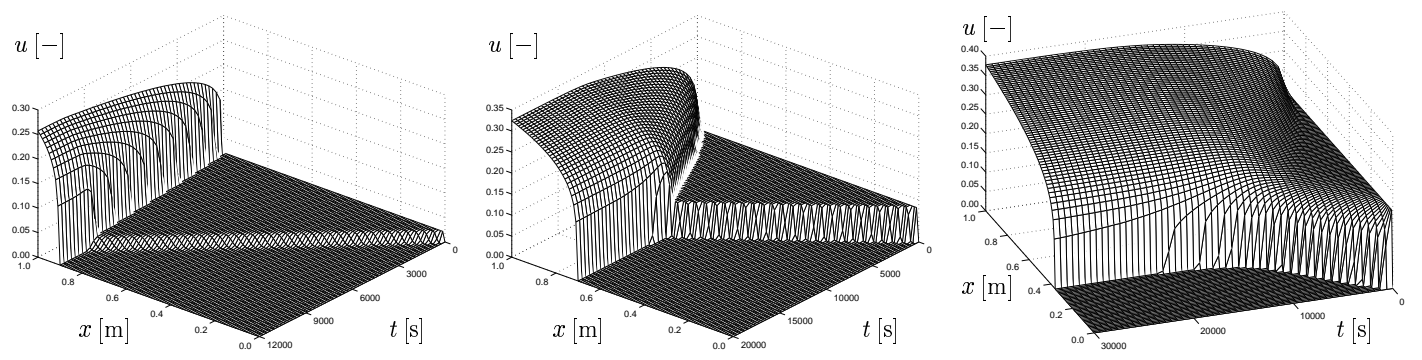

FiguRE 6. Example 1: Simulation of batch settling of an initially homogeneous suspension with $u_{0}=0.02$ (left), $u_{0}=0.08$ (middle) and $u_{0}=0.20$.

6.2. Example 1: Batch settling. To illustrate the material behaviour of the suspension, we present in Figure 6 three simulations of the settling of an initially homogeneous suspension at initial concentrations $u_{0}=0.02,0.08$ and 0.2 in a closed column (for which all $Q$ 's and $q$ 's vanish) of height $L=1 \mathrm{~m}$. We employ the explicit numerical method (3.1), $\Delta x=L / 500$, and $\lambda=20 \mathrm{~s} / \mathrm{m}$. In the first two cases, we have $a\left(u_{0}\right)=0$, and the suspension-clear liquid interface propagates as a sharp shock and the transition between the region of initial concentration and the sediment rising from below is sharp, while in the third case transitions are continuous. In all three cases, a stationary sediment is forming. In Figure 6 and all subsequent three-dimensional plots, the visual grid used to represent the solution is much coarser than the computational.

\subsection{Numerical simulations of Model 1.}

6.3.1. Example 2: Variation of discharge and overflow rates. The four simulations shown in Figure 7 have been computed using the unique feed flux $q_{\mathrm{F}} u_{\mathrm{F}}=\left(q_{\mathrm{R}}-q_{\mathrm{L}}\right) u_{\mathrm{F}}$ with $q_{\mathrm{F}}=1.25 \times 10^{-5} \mathrm{~m} / \mathrm{s}$ and $u_{\mathrm{F}}=0.08$, but by using four different "splits" of the feed rate into the discharge and overflow rates attained by varying the parameter $\nu \in[0,1]$ in $q_{\mathrm{R}}=\nu q_{\mathrm{F}}$ and $q_{\mathrm{L}}=-(1-\nu) q_{\mathrm{F}}$. In all four simulations, solving the transient equations for sufficiently large times apparently leads to a stationary solution. The numerical scheme is the explicit one (3.1) with $\lambda=40 \mathrm{~s} / \mathrm{m}$, and for this and all other numerical simulations of Model 1, we choose $\Delta x=1 / 300 \mathrm{~m}$.

In the upper left plot of Figure 7, we set $\nu=1$, i.e. the vessel is closed at the top and opened at the bottom, with the volume feed rate equalling the discharge rate. We see that the feed suspension is immediately diluted upon entering the vessel but attains its original concentration, 0.08 , again when passing the discharge level. No compression region occurs. 

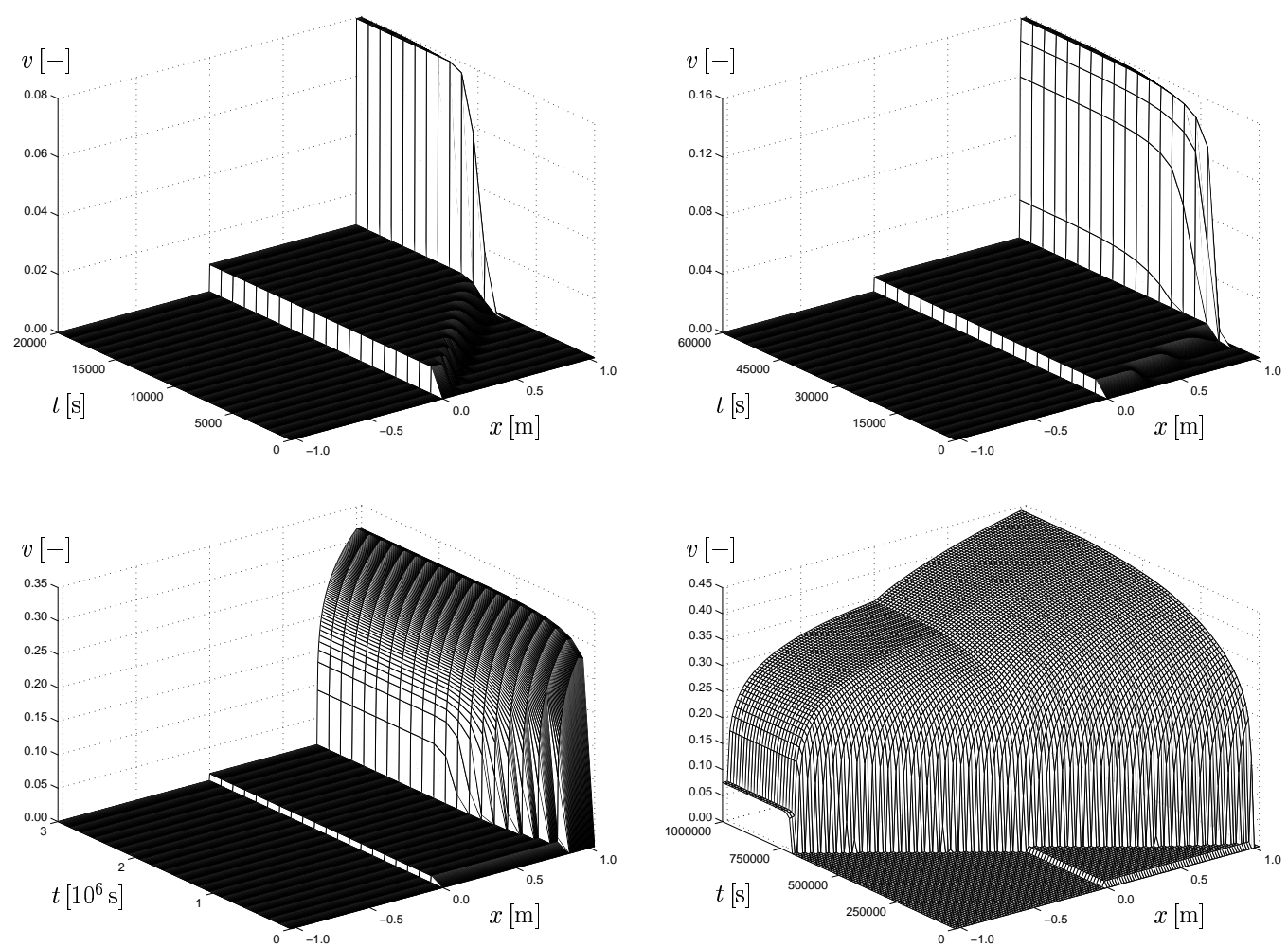

Figure 7. Example 2: Simulation of filling up a cylindrical clarifier-thickener with $u_{\mathrm{F}}=0.08, q_{\mathrm{F}}=1.25 \times 10^{-5} \mathrm{~m} / \mathrm{s}, q_{\mathrm{R}}=\nu q_{\mathrm{F}}$ and $q_{\mathrm{L}}=-(1-\nu) q_{\mathrm{F}}$ for $\nu=1$ (top left), $\nu=0.5$ (top right), $\nu=0.25$ (bottom left) and $\nu=0$ (bottom right).

For $\nu=0.5$ (top right plot of Figure 7), we obtain a very thin sediment layer at the bottom, and the discharge concentration is 0.16 , twice the feed concentration. The solution qualitatively agrees with that for $\nu=0.25$ (bottom left plot of Figure 7). However, for $\nu=0.25$ the final discharge concentration is $0.32=4 u_{\mathrm{F}}$, and the sediment layer is appreciable. The stationary solutions attained in these cases correspond to steady-state solutions of Case 1 (conventional operation).

Finally, we take $\nu=0$, i.e., the vessel is closed at its bottom. The corresponding solution is shown in the bottom right plot of Figure 7 . We observe that the feed suspension is at first immediately diluted upon entering the thickening zone. The material forms a compressible sediment layer at the bottom. This layer rises at nearly constant speed, breaks into the clarification zone, and finally produces an overflow at constant concentration 0.08 , which is just the feed concentration. (Note that this kind of steady state is not included in the analysis of Section 5.)

6.3.2. Example 3: Transitions between approximate steady states. We now utilize the examples of Section 5.4 to design a long-time numerical example in which the parameters for the timedependent Model 1 are chosen in such a way that the pre-determined steady states may be attained. This example and Example 4 (for Model 2) are solved by the semi-implicit method (3.4) with $\lambda=$ $4000 \mathrm{~s} / \mathrm{m}$. We consider the constant flow velocities $q_{\mathrm{R}}=2.5 \times 10^{-6} \mathrm{~m} / \mathrm{s}$ and $q_{\mathrm{L}}=-1.0 \times 10^{-5} \mathrm{~m} / \mathrm{s}$. The feed concentration $u_{\mathrm{F}}$ is varied in a stepwise fashion as follows:

$$
u_{\mathrm{F}}(t)=\left\{\begin{array}{llll}
0.086 & \text { for } 0 \leq t \leq t_{1}:=4.0 \times 10^{7} \mathrm{~s}, & 0.088 & \text { for } t_{2}<t \leq t_{3}:=9.5 \times 10^{7} \mathrm{~s} \\
0.08 & \text { for } t_{1}<t \leq t_{2}:=6.0 \times 10^{7} \mathrm{~s}, & 0 & \text { for } t>t_{3}
\end{array}\right.
$$

The initial stage of the fill-up process is shown in the top left plot of Figure 8 , while the complete solution for the first time interval $\left[0, t_{1}\right]$ is plotted in the top right plot of Figure 8 . We observe that the feed propagates as a rarefaction wave into the thickening zone, and that a sediment layer is built 

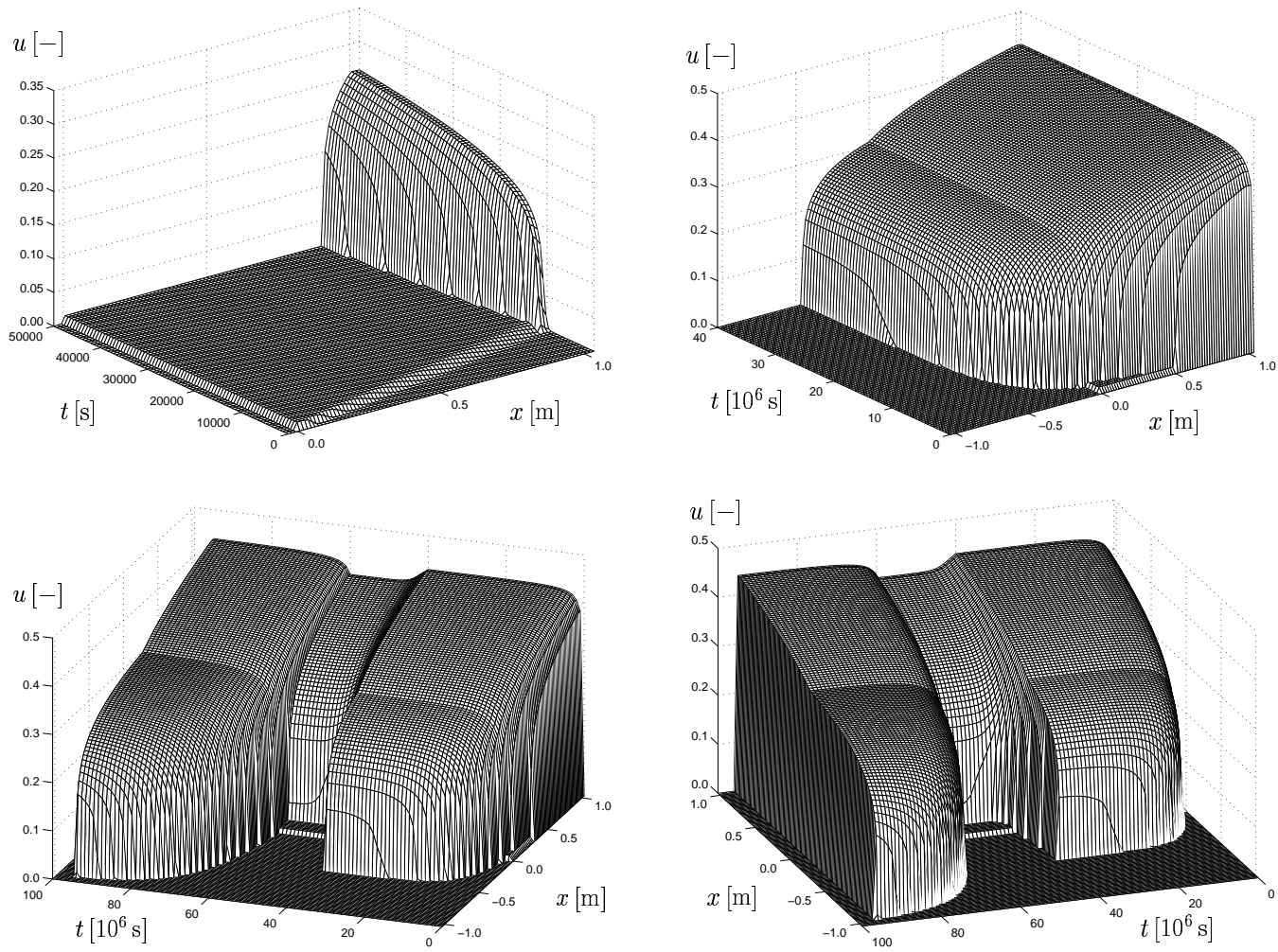

Figure 8. Example 3: Simulations of the fillup and transitions between steady states in a cylindrical clarifier-thickener (Vessel 1): filling up a cylindrical clarifierthickener up to steady state with $u_{\mathrm{F}}=0.086$ (initial stages: top left, complete process: top right), and two different views of the complete simulation with successive changes of $u_{\mathrm{F}}$ from 0.086 to $0.08,0.088$ and 0 (bottom plots).

up, which rises above the feed level. The interesting point is that the numerical solution becomes stationary after the very long simulated time of about $3.0 \times 10^{7} \mathrm{~s}$, which corresponds to roughly one year, and the stationary solution closely approximates the steady-state profile corresponding to the same values of $q_{\mathrm{L}}, q_{\mathrm{R}}$ and $u_{\mathrm{F}}$ plotted in Figure 4 . In particular, the numerical value of the overflow concentration remains zero, and the solution value assumed at $w=x_{\mathrm{R}}$ equals 0.42997 . Thus, we have reason to believe that this steady-state solution is indeed the limit attained by the entropy weak solution for these parameters, at least for $t \rightarrow \infty$; whether the steady state is reached even in finite time would be a further going question.

At the simulated time $t=t_{1}$, we reduce $u_{\mathrm{F}}$ to a value that in combination with those of $q_{\mathrm{L}}$ and $q_{\mathrm{R}}$ once again corresponds to a steady state plotted in Figure 4, but this time to one of conventional operation. The bottom left and right plots of Figure 8 indicate that also this steady state seems to be attained by the transient solution. In particular, the hindered settling region becomes visible again. Shortly before $t=t_{2}$, the numerical solution value at $x=x_{\mathrm{R}}$ equals 0.40001 .

At $t=t_{2}$, we increase $u_{\mathrm{F}}$ to 0.088 , and we observe that the simulation converges again to the corresponding steady state of Figure 4 . Shortly before the solution becomes stationary, at $t=t_{3}$, we switch off the feed by setting $u_{\mathrm{F}}=0$, and the clarifier-thickener unit empties rapidly.

Although the operations involved in this run-filling up, transitions between steady states and emptying of a clarifier-thickener - are typical control actions, practitioners would, of course, accelerate the fill-up by closing the unit $[15,16]$. The main intention behind our example is, however, to illustrate that the model apparently converges to steady-state solutions. 

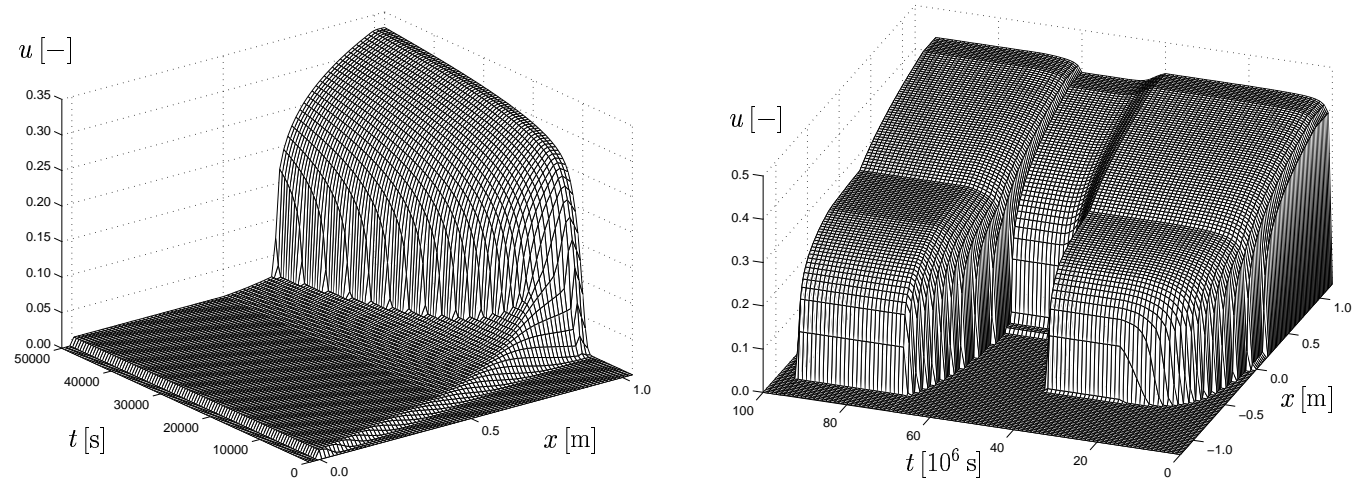

Figure 9. Example 4: Simulation of transitions between steady states in Vessel 2: plot of the initial fill-up stage (left) and long-time simulation (right).

\begin{tabular}{rcccccc}
\hline \multicolumn{3}{c}{$t=200000 \mathrm{~s}$} & \multicolumn{2}{c}{$t=1000000 \mathrm{~s}$} & \multicolumn{2}{c}{$t=2000000 \mathrm{~s}$} \\
$J=\frac{1 \mathrm{~m}}{\Delta x}$ & $L^{1}$ error & conv. rate & $L^{1}$ error & conv. rate & $L^{1}$ error & conv. rate \\
\hline 10 & $1.429 \mathrm{e}-2$ & & $2.301 \mathrm{e}-2$ & & $2.857 \mathrm{e}-2$ & \\
20 & $7.092 \mathrm{e}-3$ & 1.010 & $1.115 \mathrm{e}-2$ & 1.004 & $1.424 \mathrm{e}-2$ & 1.005 \\
40 & $3.475 \mathrm{e}-3$ & 1.029 & $5.666 \mathrm{e}-3$ & 1.017 & $7.026 \mathrm{e}-3$ & 1.019 \\
80 & $1.691 \mathrm{e}-3$ & 1.039 & $2.758 \mathrm{e}-3$ & 1.038 & $3.425 \mathrm{e}-3$ & 1.037 \\
100 & $1.338 \mathrm{e}-3$ & 1.048 & $2.176 \mathrm{e}-3$ & 1.063 & $2.704 \mathrm{e}-3$ & 1.059 \\
160 & $8.004 \mathrm{e}-4$ & 1.094 & $1.306 \mathrm{e}-3$ & 1.087 & $1.623 \mathrm{e}-3$ & 1.085 \\
200 & $6.225 \mathrm{e}-4$ & 1.126 & $1.015 \mathrm{e}-3$ & 1.129 & $1.263 \mathrm{e}-3$ & 1.124 \\
\hline
\end{tabular}

TABLE 2. Approximate $L^{1}$ errors for the numerical solution of Example 3.

6.4. Example 4: Numerical simulation of Model 2. Finally, we repeat the simulation of Example 3, that is, we use again the function $u_{\mathrm{F}}(t)$ defined by $(6.1)$, but now we consider Model 2, Vessel 2 drawn in Figure 5, and select $Q_{\mathrm{L}}=-1.0 \times 10^{-5} \mathrm{~m}^{3} / \mathrm{s}$ and $Q_{\mathrm{R}}=2.5 \times 10^{-6} \mathrm{~m}^{3} / \mathrm{s}$. Model 2 enforces a CFL condition involving a factor $\max S(x) / \min S(x)$, which equals 25 here, so we have to decrease accuracy to maintain acceptable computation time. We here chose $\lambda=100 \mathrm{~s} / \mathrm{m}$ and $\Delta x=1 / 50 \mathrm{~m}$ for the long-time run shown in the right plot of Figure 9 but $\Delta x=1 / 300 \mathrm{~m}$ for the short simulated period in the left plot.

We observe that the concentration profiles slightly reflects the thickener geometry, although due to the diffusion term, this effect is less pronounced than for the same model without compression (i.e., for $A \equiv 0$ ), see $[23,24]$. First, observe the difference between the left plot of Figure 9 and the corresponding simulation for Model 1 and Vessel 1 in the upper left plot of Figure 8. The conical cross-section of the lower part of the thickening zone causes a continuous variation of the concentration in the initial hindered settling region and accelerates the fill-up process.

Of course, a variable cross-sectional area $S(x)$ complicates the discussion of steady states but also offers new design opportunities [16]. Here, Vessel 2 does no longer admit a steady state for $u_{\mathrm{F}}=0.088$ with $u_{\mathrm{E}}=0$. In fact, we observe in the right plot of Figure 9 that a stationary profile is attained with non-zero overflow concentration. Rather, the numerical values attained are overflow and underflow concentrations $u\left(x_{\mathrm{L}}^{-}\right)=0.00121$ and $u\left(x_{\mathrm{R}}^{+}\right)=0.43293$. The stationary profile is probably a steady state with non-zero effluent concentration (which is not included in the analysis of Section 5, as aren't any other steady states for Model 2).

6.5. Comments on the numerical results. First of all, we mention that our numerical results, including test runs with coarser discretizations (not shown here), suggest that the scheme indeed converges to solutions for which $A(u)$ is continuous across $x_{\mathrm{L}}$ and $x_{\mathrm{R}}$, as required by 
condition (D.4). However, it should be emphasized that our scheme does not possess a built-in mechanism to enforce this property. As emphasized before, a rigorous proof for the convergence of the scheme towards a solution satisfying (D.4) is still an open problem, and it might be that one even has to modify the scheme to ensure this property. This requires a deeper numerical analysis, which we defer to another paper.

Furthermore, the accuracy and convergence rate of the numerical scheme used herein may be of interest. To this end, we measured approximate $L^{1}$ errors for the simulation of Example 3 by measuring the difference $\left\|u^{\Delta}(\cdot, t)-u^{\mathrm{ref}}(\cdot, t)\right\|_{L^{1}(-1.1 \mathrm{~m}, 1.1 \mathrm{~m})}$ for a number of discretizations $(\Delta x, \Delta t=\lambda \Delta x)$ at $t=200000 \mathrm{~s}, t=1000000 \mathrm{~s}$, and $t=2000000 \mathrm{~s}$, where $u^{\Delta}$ is the numerical solution obtained with $\Delta x=1 \mathrm{~m} / J, J=10,20,40,80,100,160,200$, and $u^{\text {ref }}$ is a high-accuracy reference solution with $J=1600$, see Table 2. In all cases, the semi-implicit scheme (3.4) with the parameter $\lambda=4000 \mathrm{~s} / \mathrm{m}$ was used.

We observe that the approximate $L^{1}$ convergence rates are slightly larger than but close to one. This is consistent with the formal first-order accuracy of the time discretization and of the discretization of the convective fluxes. Similar approximately linear convergence has been observed for the explicit version (3.1) of the scheme applied to a slightly simpler equation that does not involve a discontinuous parameter in the diffusion term in [55], and for the application of the explicit scheme to the initial-boundary value problem of batch settling of a flocculated suspension in [18], which does not involve a discontinuous parameter at all. It should be pointed out that observed convergence rates substantially depend on the parameters and numerical examples chosen. For example, similar approximate $L^{1}$ tables for the explicit scheme (3.1) applied to the first-order clarifier-thickener model (obtained by setting $A \equiv 0$ ) are presented in [25]. It turns out that when approximate $L^{1}$ errors are measured at times when the solution includes nonstationary 'hyperbolic' discontinuities (shocks), then the observed $L^{1}$ convergence rate measured on a succession of grids may fall substantially below one.

To put these observations into the proper perspective, let us mention that a theoretical estimate of the rate of convergence of the numerical scheme presented herein is outside current theory, even in the case of smooth coefficients. However, our prime motivation behind advancing the scheme (3.1) (and its semi-implicit variant (3.4)) was to use it as a constructive tool for the well-posedness analysis, and to employ it for simulations to illustrate the mathematical analysis. Clearly, we do not propose (3.1) or (3.4) as the optimal scheme for simulations in practice. For that purpose the scheme should be upgraded to formal second-order both in time and in space accuracy, which can be attained, for example, by combining flux correction and Strang-type operator splitting between the hyperbolic and parabolic portions of the problem. We pursue this further in [26].

Finally, there are conceivable alternative schemes for the clarifier-thickener model that seem worth exploring. For example, one could combine the very efficient front tracking method introduced in [19] for the hyperbolic portion and combine it with finite differencing of the diffusion term in an operator splitting procedure. Alternatively, the relaxation scheme used in [21] for the simulation of the first-order clarifier-thickener model could be extended to handle the second-order degenerate diffusion term accounting for sediment compressibility (as in [29]). Recent numerical schemes for strongly degenerate parabolic equations that can possibly be extended to the clarifierthickener model also include the local discontinuous Galerkin method [33] and diffusive kinetic BGK approximations [3, 11].

\section{ACKNOWLEDGments}

This work has been supported by the Collaborative Research Center (Sonderforschungsbereich) 404 at the University of Stuttgart, the BeMatA program of the Research Council of Norway, and the European network HYKE, funded by the EC as contract HPRN-CT-2002-00282. We are grateful to the referees for valuable comments that resulted in a number of improvements in this paper. 


\section{REFERENCES}

[1] Adimurthi, J. Jaffré, and G.D. Veerappa Gowda, Godunov-type methods for conservation laws with a flux function discontinuous in space, SIAM J. Numer. Anal., 42 (2004), pp. 179-208.

[2] D. Amadori, L. Gosse, And G. Guerrac, Godunov-type approximation for a general resonant balance law with large data, J. Diff. Eqns., 198 (2004), pp. 233-274.

[3] D. Aregba-Driollet, R. Natalini, and S. TANG, Explicit diffusive kinetic schemes for nonlinear degenerate parabolic systems, Math. Comp., 73 (2004), pp. 63-94.

[4] A.A.A. Aziz, R.G. De Kretser, D.R. Dixon, and P.J. Scales, The characterisation of slurry dewatering, Wat. Sci. Tech., 41 (8) (2000), pp. 9-16.

[5] P. Baiti and H.K. Jenssen, Well-posedness for a class of $2 \times 2$ conservation laws with $L^{\infty}$ data, J. Diff. Eqns., 140 (1997), pp. 161-185.

[6] N.G. Barton, C.-H. Li, And S.J. Spencer, Control of a surface of discontinuity in continuous thickeners, J. Austral. Math. Soc. Ser. B, 33 (1992), pp. 269-289.

[7] M. Bendahmane And K.H. Karlsen, Renormalized entropy solutions for quasilinear anisotropic degenerate parabolic equations, SIAM J. Math. Anal., 36 (2) (2004), pp. 405-422.

[8] P. Bénilan And H. Touré, Sur l'équation générale $u_{t}=a\left(\cdot, u, \phi(\cdot, u)_{x}\right)_{x}+v$ dans $L^{1}$. II. Le problème d'évolution, Ann. Inst. H. Poincaré Anal. Non Linéaire, 12 (1995), pp. 727-761.

[9] S. Berres, R. Bürger, And K.H. Karlsen, Central schemes and systems of conservation laws with discontinuous coefficients modeling gravity separation of polydisperse suspensions, J. Comp. Appl. Math., 164-165 (2004), pp. 53-80.

[10] S. Berres, R. Bürger, K.H. Karlsen, and E.M. Tory, Strongly degenerate parabolic-hyperbolic systems modeling polydisperse sedimentation with compression, SIAM J. Appl. Math., 64 (2003), pp. 41-80.

[11] F. Bouchut, F.R. Guarguaglini, and R. Natalini, Diffusive BGK approximations for nonlinear multidimensional parabolic equations, Indiana Univ. Math. J., 49 (2000), pp. 723-749.

[12] F. Bouchut and F. James, One-dimensional transport equations with discontinuous coefficients, Nonlinear Anal. TMA, 32 (1998), pp. 891-933.

[13] R. Bürger, M.C. Bustos, And F. Concha, Settling velocities of particulate systems: 9. Phenomenological theory of sedimentation processes: Numerical simulation of the transient behaviour of flocculated suspensions in an ideal batch or continuous thickener, Int. J. Mineral Process., 55 (1999), pp. 267-282.

[14] R. Bürger and F. Concha, Settling velocities of particulate systems: 12. Batch centrifugation of flocculated suspensions, Int. J. Mineral Process., 63 (2001), pp. 115-145.

[15] R. Bürger, F. Concha, And F.M. Tiller, Applications of the phenomenological theory to several published experimental cases of sedimentation processes, Chem. Eng. J., 80 (2000), pp. 105-117.

[16] R. Bürger, J.J.R. Damasceno, and K.H. Karlsen, A mathematical model for batch and continuous thickening in vessels with varying cross section, Int. J. Mineral Process., 73 (2004), pp. 183-208.

[17] R. Bürger, S. Evje, K.H. Karlsen, And K.-A. Lie, Numerical methods for the simulation of the settling of flocculated suspensions, Chem. Eng. J., 80 (2000), pp. 91-104.

[18] R. BÜRGER AND K.H. KARLSEN, On some upwind schemes for the phenomenological sedimentationconsolidation model, J. Eng. Math., 41 (2001), pp. 145-166.

[19] R. Bürger, K.H. Karlsen, C. Klingenberg, and N.H. Risebro, A front tracking approach to a model of continuous sedimentation in ideal clarifier-thickener units, Nonlin. Anal. Real World Appl., 4 (2003), pp. 457481.

[20] R. Bürger, K.H. Karlsen, S. Mishra, and J.D. Towers, On conservation laws with discontinuous flux. Preprint, Institute of Applied Analysis and Numerical Simulation, University of Stuttgart, 2003.

[21] R. Bürger, K.H. Karlsen, and N.H. Risebro, A relaxation scheme for continuous sedimentation in ideal clarifier-thickener units, Comp. Math. Appl., to appear.

[22] R. Bürger, K.H. Karlsen, N.H. Risebro, and J.D. Towers, Numerical methods for the simulation of continuous sedimentation in ideal clarifier-thickener units, Int. J. Mineral Process., 73 (2004), pp. 209-228.

[23] R. Bürger, K.H. Karlsen, N.H. Risebro, and J.D. Towers, Monotone difference approximations for the simulation of clarifier-thickener units, Comput. Visual. Sci., 6 (2004), pp. 83-91.

[24] R. Bürger, K.H. Karlsen, N.H. Risebro, And J.D. Towers, On a model for continuous sedimentation in vessels with discontinuously varying cross-sectional area. In: T.Y. HOU AND E. TADMOR (eds.), Hyperbolic Problems: Theory, Numerics, Applications. Proceedings of the Ninth International Conference on Hyperbolic Problems held in CalTech, Pasadena, March 25-29, 2002. Springer-Verlag, Berlin (2003), pp. 397-406.

[25] R. Bürger, K.H. Karlsen, N.H. Risebro, and J.D. Towers, Well-posedness in BVt and convergence of a difference scheme for continuous sedimentation in ideal clarifier-thickener units, Numer. Math., 97 (2004), pp. 25-65.

[26] R. Bürger, K.H. KARlSen, AND J.D. Towers, High resolution schemes for continuous sedimentation in ideal clarifier-thickener units. In preparation.

[27] R. Bürger, W.L. Wendland, And F. Concha, Model equations for gravitational sedimentation-consolidation processes, Z. Angew. Math. Mech., 80 (2000), pp. 79-92.

[28] J. CARRILlo, Entropy solutions for nonlinear degenerate problems, Arch. Rational Mech. Anal., 147 (1999), pp. 269-361. 
[29] F. Cavalli, G. Naldi, And G. Toscani, Relaxation methods for the sedimentation of polydisperse suspensions of spheres, Preprint, Milano, Italy, 2002.

[30] J.P. Chancelier, M. Cohen de Lara, and F. PaCard, Analysis of a conservation PDE with discontinuous flux: a model of settler, SIAM J. Appl. Math., 54 (1994), pp. 954-995.

[31] G.-Q. Chen And E. DiBenedetto, Stability of entropy solutions to the Cauchy problem for a class of nonlinear hyperbolic-parabolic equations, SIAM J. Math. Anal., 33 (2001), pp. 751-762.

[32] G.-Q. Chen and B. Perthame, Well-posedness for non-isotropic degenerate parabolic-hyperbolic equations, Ann. Inst. H. Poincaré Anal. Non Linéaire, 20 (2003), pp. 645-668.

[33] B. Cockburn And C.-W. Shu, The local discontinuous Galerkin method for time-dependent convectiondiffusion problems, SIAM J. Numer. Anal., 35 (1998), pp. 2440-2463.

[34] F. Concha, A. Barrientos, And M.C. Bustos, Phenomenological model of High Capacity Thickening. In: Proc. of the 19th Int. Mineral Processing Congress (XIX IMPC), San Francisco, USA (1995), Ch. 14, pp. 75-79.

[35] R. Courant, K. Friedrichs, AND H. LewY, Über die partiellen Differenzengleichungen der mathematischen Physik, Math. Annalen, 100 (1928), pp. 28-74.

[36] M. G. CRandall And A. Majda, Monotone difference approximations for scalar conservation laws, Math. Comp., 34 (1980), pp. 1-21.

[37] M.G. Crandall and L. Tartar, Some relations between nonexpansive and order preserving mappings, Proc. Amer. Math. Soc., 78 (1980), pp. 385-390.

[38] R.G. De Kretser, S.P. Usher, P.J. Scales, D.V. Boger, and K.A. Landman, Rapid filtration measurement of dewatering design and optimization parameters, AIChE J., 47 (2001), pp. 1758-1769.

[39] S. DieHL, On scalar conservation laws with point source and discontinuous flux function, SIAM J. Math. Anal., 26 (1995), pp. 1425-1451.

[40] S. DieHL, A conservation law with point source and discontinuous flux function modelling continuous sedimentation, SIAM J. Appl. Math., 56 (1996), pp. 388-419.

[41] S. DIEHL, Dynamic and steady-state behaviour of continuous sedimentation, SIAM J. Appl. Math., 57 (1997), pp. 991-1018.

[42] S. DieHL, On boundary conditions and solutions for ideal clarifier-thickener units, Chem. Eng. J., 80 (2000), pp. 119-133.

[43] S. DieHL, Operating charts for continuous sedimentation I: Control of steady states, J. Eng. Math., 41 (2001), pp. 117-144.

[44] B. Engquist And S. Osher, One-sided difference approximations for nonlinear conservation laws, Math. Comp., 36 (1981), pp. 321-351.

[45] P. Garrido, R. Bürger, And F. Concha, Settling velocities of particulate systems: 11. Comparison of the phenomenological sedimentation-consolidation model with published experimental results, Int. J. Mineral Process., 60 (2000), pp. 213-227.

[46] T. Gimse, Conservation laws with discontinuous flux functions, SIAM J. Math. Anal. 24 (1993), pp. 279-289.

[47] T. Gimse And N.H. Risebro, Riemann problems with a discontinuous flux function. In: Proc. 3rd Conf. Hyp. Problems, Uppsala, Sweden, 1990.

[48] T. Gimse And N.H. Risebro, Solution of the Cauchy problem for a conservation law with a discontinuous flux function, SIAM J. Math. Anal., 23 (1992), pp. 635-648.

[49] J.M.-K. Hong, Part I: An extension of the Riemann problems and Glimm's method to general systems of conservation laws with source terms. Part II: A total variation bound on the conserved quantities for a generic resonant nonlinear balance laws, $\mathrm{PhD}$ thesis, University of California, Davis, 2000.

[50] J. JAFFRÉ, Numerical calculation of the flux across an interface between two rock types of a porous medium for a two-phase flow. In: Hyperbolic Problems: Theory, Numerics, Applications (Stony Brook, NY, 1994), World Sci. Publishing, River Edge, NJ, 1996, pp. 165-177.

[51] E.F. KaAsschieter, Solving the Buckley-Leverett equation with gravity in a heterogeneous porous medium, Comput. Geosci., 3 (1999), pp. 23-48.

[52] K.H. Karlsen, C. Klingenberg, and N.H. Risebro, A relaxation scheme for conservation laws with discontinuous coefficients, Math. Comp., 73 (2004), pp. 1235-1259.

[53] K.H. Karlsen AND N.H. Risebro, On the uniqueness and stability of entropy solutions of nonlinear degenerate parabolic equations with rough coefficients, Discr. Cont. Dyn. Syst., 9 (2003), pp. 1081-1104.

[54] K.H. Karlsen, N.H. Risebro, AND J.D. Towers, On a nonlinear degenerate parabolic transport-diffusion equation with a discontinuous coefficient, Electron. J. Diff. Eqns., 2002 (2002), No. 93, pp. 1-23.

[55] K.H. Karlsen, N.H. Risebro, and J.D. Towers, On an upwind difference scheme for degenerate parabolic convection-diffusion equations with a discontinuous coefficient, IMA J. Numer. Anal., 22 (2002), pp. 623-664.

[56] K.H. Karlsen, N.H. Risebro, And J.D. Towers, $L^{1}$ stability for entropy solutions of nonlinear degenerate parabolic convection-diffusion equations with discontinuous coefficients, Skr. K. Nor. Vid. Selsk, 2003, 49 pp.

[57] K.H. KarlSen And J.D. Towers, Convergence of the Lax-Friedrichs scheme and stability for conservation laws with a discontinuous space-time dependent flux, Chin. Ann. Math. Ser. B, to appear.

[58] R.A. Klausen and N.H. Risebro, Stability of conservation laws with discontinuous coefficients, J. Diff. Eqns., 157 (1999), pp. 41-60.

[59] C. Klingenberg and N.H. Risebro, Convex conservation laws with discontinuous coefficients, Existence, uniqueness and asymptotic behavior. Comm. Partial Diff. Eqns., 20 (1995), pp. 1959-1990. 
[60] C. Klingenberg And N. H. Risebro, Stability of a resonant system of conservation laws modeling polymer flow with gravitation, J. Diff. Eqns., 170 (2001), pp. 344-380.

[61] S.N. KRUŽKov, First order quasi-linear equations in several independent variables, Math. USSR Sbornik, 10 (1970), pp. 217-243.

[62] G.J. KYNCH, A theory of sedimentation, Trans. Farad. Soc., 48 (1952), pp. 166-176.

[63] D.R. Lester, Colloidal Suspension Dewatering Analysis, PhD Thesis, Department of Chemical Engineering, University of Melbourne, Australia, 2002.

[64] O. Lev, E. Rubin, And M. Sheintuch, Steady state analysis of a continuous clarifier-thickener system, AIChE J., 32 (1986), pp. 1516-1525.

[65] L.W. Lin, B.J. Temple, And J.H. WANG, A comparison of convergence rates for Godunov's method and Glimm's method in resonant nonlinear systems of conservation laws, SIAM J. Numer. Anal., 32 (1995), pp. 824-840.

[66] L.W. Lin, B.J. Temple, And J.H. Wang, Suppression of oscillations in Godunov's method for a resonant non-strictly hyperbolic system, SIAM J. Numer. Anal., 32 (1995), pp. 841-864.

[67] W.K. Lyons, Conservation laws with sharp inhomogeneities, Quart. Appl. Math., 40 (1982/83), pp. $385-393$.

[68] C. Mascia, A. Porretta, and A. Terracina, Nonhomogeneous Dirichlet problems for degenerate parabolichyperbolic equations, Arch. Rational Mech. Anal., 163 (2002), pp. 87-124.

[69] A. Michel and J. Vovelle, Entropy formulation for parabolic degenerate equations with general Dirichlet boundary conditions and application to the convergence of FV methods, SIAM J. Numer. Anal., 41 (2003), pp. 2262-2293

[70] S. MishrA, Convergence of Upwind Finite Difference Schemes for a scalar conservation law with indefinite discontinuities in the flux function. Preprint, 2002.

[71] J. MolenaAR, Entropy conditions for heterogeneity induced shocks in two-phase flow problems. In: A.P. Bourgeat, C. Carasso, S. Luckhaus, And A. Mikelic (eds.), Mathematical Modelling of Flow Through Porous Media, World Scientific, Singapore, 1995.

[72] D.N. Ostrov, Viscosity solutions and convergence of monotone schemes for synthetic aperture radar shapefrom-shading equations with discontinuous intensities, SIAM J. Appl. Math., 59 (1999), pp. 2060-2085.

[73] D.N. Ostrov, Solutions of Hamilton-Jacobi equations and scalar conservation laws with discontinuous spacetime dependence, J. Diff. Eqns., 182 (2002), pp. 51-77.

[74] J.F. Richardson and W.N. Zaki, Sedimentation and fluidization: Part I, Trans. Instn. Chem. Engrs. (London), 32 (1954), pp. 35-53.

[75] N. Seguin And J. Vovelle, Analysis and approximation of a scalar conservation law with a flux function with discontinuous coefficients, Math. Models Meth. Appl. Sci., 13 (2003), pp. 221-257.

[76] B. Temple, Global solution of the Cauchy problem for a class of $2 \times 2$ nonstrictly hyperbolic conservation laws, Adv. Appl. Math., 3 (1982), pp. 335-375.

[77] J.D. Towers, Convergence of a difference scheme for conservation laws with a discontinuous flux, SIAM J. Numer. Anal., 38 (2000), pp. 681-698.

[78] J.D. Towers, A difference scheme for conservation laws with a discontinuous flux: the nonconvex case, SIAM J. Numer. Anal., 39 (2001), pp. 1197-1218.

[79] S.P. Usher, R.G. De Kretser, and P.J. Scales, Validation of a new filtration technique for dewaterability characterization, AIChE J., 47 (2001), pp. 1561-1570.

[80] C.J. van Duijn, M.J. De Neef, and J. MolenaAR, Effects of capillary forces on immiscible two-phase flow in strongly heterogeneous porous media, Transp. Porous Media, 21 (1995), pp. 71-93.

[81] L.B. Verdickt, T.V. Voitovich, S. Vandewalle, K. Lust, I.Y. Smets, and J.F. Van Impe, Role of the diffusion coefficient in one-dimensional convection-diffusion models for sedimentation/thickening in secondary settling tanks, Math. Comp. Mod. Dyn. Syst., to appear.

[82] A.I. VOLPERT, Generalized solutions of degenerate second-order quasilinear parabolic and elliptic equations, Adv. Diff. Eqns., 5 (2000), pp. 1493-1518.

[83] A.I. Vol'pert And S.I. Hudjaev, Cauchy's problem for degenerate second order quasilinear parabolic equations, Math. USSR Sb., 7 (1969), pp. 365-387.

[84] Z. WU AND J. YIN, Some properties of functions in $B V_{x}$ and their applications to the uniqueness of solutions for degenerate quasilinear parabolic equations, Northeastern Math. J., 5 (1989), pp. 395-422.

[85] Y.C. Yortsos And J. Chang, Capillary effects in steady-state flow in heterogeneous cores, Transp. Porous Media, 5 (1990), pp. 399-420. 